Supporting Information

\title{
Indium-Mediated Regio- and Chemoselective Synthesis of a-Hydroxylalkyl Allenic Esters and Gold-Catalyzed Cyclizations to Ethyl 2-Naphthoate Derivatives
}

\author{
Chansoo Park and Phil Ho Lee* \\ National Research Laboratory for Catalytic Organic Reaction, \\ Department of Chemistry and Institute for Molecular Science and Fusion Technology, \\ Kangwon National University, Chunchon 200-701, Repubic of Korea
}

phlee@kangwon.ac.kr

Table of Contents

Experimental procedure and data of compounds S2-S14

ReferencesS15

${ }^{1} \mathrm{H}$ and ${ }^{13} \mathrm{C}$ NMR spectra for compoundsS16-S67 


\section{Experimental Section}

Typical experimental procedure of the reaction of benzaldehyde with indium and ethyl 4-bromobutynoate: Ethyl 4-bromobutynoate ( $95.5 \mu \ell, 0.75 \mathrm{mmol})$ was added to suspension of indium (57.4 mg, $0.5 \mathrm{mmol}$ ) and LiI (200.8 mg, $1.5 \mathrm{mmol})$ in DMF (2.0 $\mathrm{mL}$ ). After being stirred for $30 \mathrm{~min}$ at room temperature under a nitrogen atmosphere, benzaldehyde (50.7 $\mu$, $0.5 \mathrm{mmol}$ ) was added and then. After $5 \mathrm{~h}$, the reaction mixture was quenched with saturated $\mathrm{NaHCO}_{3}(20 \mathrm{~mL})$. The aqueous layer was extracted with diethyl ether (3 x $20 \mathrm{~mL}$ ). The combine organic layers were washed with brine (20 mL). The resulting organic layers were dried over $\mathrm{MgSO}_{4}$, filtered and concentrated under a reduced pressure. The residue was purified by silica gel column chromatography (ethyl acetate : hexane $=1: 5$ ) to give 2-(hydroxyphenylmethyl)buta-2,3-dienoic acid ethyl ester (93.8 mg, 86\%).<smiles>C=CC(C(=O)OCC)C(O)C(=C)C</smiles>

5e

2-(Hydroxyphenylmethyl)buta-2,3-dienoic acid ethyl ester (5e) ${ }^{\mathbf{1}}{ }^{1} \mathrm{H}$ NMR (400 MHz, $\left.\mathrm{CDCl}_{3}\right) \delta$ 7.42-7.26 (m, 5H), $5.61(\mathrm{~d}, J=6.29 \mathrm{~Hz}, 1 \mathrm{H}), 5.19(\mathrm{~s}, 2 \mathrm{H}), 4.22(\mathrm{q}, J=$ $7.14 \mathrm{~Hz}, 2 \mathrm{H}), 3.49$ (d, $J=6.29 \mathrm{~Hz}, 1 \mathrm{H}), 1.24$ (t, $J=7.14 \mathrm{~Hz}, 3 \mathrm{H}) ;{ }^{13} \mathrm{C}$ NMR $(100 \mathrm{MHz}$, $\left.\mathrm{CDCl}_{3}\right) \delta 213.4,167.2,141.8,128.6,128.1,126.7,104.1,81.4,72.3,61.8,14.5 ; \mathrm{IR}$ (film) 3434, 2983, 1964, 1705, 1454, 1368, 1253, 1039, $699 \mathrm{~cm}^{-1}$.

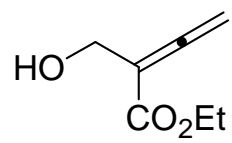

$5 \mathbf{a}$ 
2-Hydroxymethylbuta-2,3-dienoic acid ethyl ester (5a): ${ }^{1} \mathrm{H}$ NMR (400 $\left.\mathrm{MHz}, \mathrm{CDCl}_{3}\right)$ $\delta 5.25$ (s, 2H), 4.34 (d, $J=6.64 \mathrm{~Hz}, 2 \mathrm{H}), 4.25$ (q, $J=7.12 \mathrm{~Hz}, 2 \mathrm{H}), 2.54$ (t, $J=6.64 \mathrm{~Hz}$, 1H), $1.31(\mathrm{t}, J=7.12 \mathrm{~Hz}, 3 \mathrm{H}) ;{ }^{13} \mathrm{C}$ NMR $\left(100 \mathrm{MHz}, \mathrm{CDCl}_{3}\right) \delta$ 213.0, 166.9, 99.8, 79.8, 61.4, 61.1, 14.2; IR (film) 3404, 2985, 1967, 1706, 1464, 1296, 1108, 1047, $859 \mathrm{~cm}^{-1}$; HRMS (EI) : $m / z$ calcd. For $\mathrm{C}_{7} \mathrm{H}_{10} \mathrm{O}_{3}:$ 142.0630; found: 142.0627.

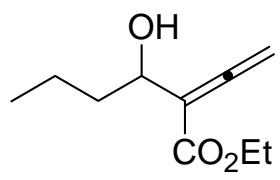

$5 \mathbf{b}$

3-Hydroxy-2-vinylidenehexanoic acid ethyl ester (5b): ${ }^{1} \mathrm{H} \mathrm{NMR}\left(400 \mathrm{MHz}, \mathrm{CDCl}_{3}\right) \delta$ 5.25 (s, 2H), 4.45-4.41 (m, 1H), 4.24 (q, $J=7.12 \mathrm{~Hz}, 2 \mathrm{H}), 2.98$ (d, $J=5.97 \mathrm{~Hz}, 1 \mathrm{H})$, 1.69-1.59 (m, 2H), 1.54-1.33 (m, 2H), 1.30 (t, $J=7.12 \mathrm{~Hz}, 3 \mathrm{H}), 0.94$ (t, $J=7.33 \mathrm{~Hz}$, 3H); ${ }^{13} \mathrm{C}$ NMR (100 MHz, $\left.\mathrm{CDCl}_{3}\right) \delta 212.7,167.6,103.6,81.0,69.5,61.6,37.8,19.4$, 14.6, 14.2; IR (film) 3455, 2960, 2873, 1964, 1708, 1254, 1066, $853 \mathrm{~cm}^{-1}$; HRMS (EI) : m/z calcd. For $\mathrm{C}_{10} \mathrm{H}_{16} \mathrm{O}_{3}$ : 184.1099; found: 184.1097.

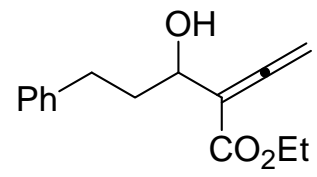

5c

3-Hydroxy-5-phenyl-2-vinylidenepentanoic acid ethyl ester (5c): ${ }^{1} \mathrm{H}$ NMR (400 $\left.\mathrm{MHz}, \mathrm{CDCl}_{3}\right) \delta$ 7.30-7.27 (m, 2H), 7.22-7.17 (m, 3H), 5.27 (s, 2H), 4.47-4.43 (m, 1H), 4.23 (q, $J=7.10 \mathrm{~Hz}, 2 \mathrm{H}$ ), 3.09 (d, $J=5.63 \mathrm{~Hz}, 1 \mathrm{H}), 2.87-2.80$ (m, 1H), 2.74-2.66 (m, 1H), 2.05-1.91 (m, 2H), 1.29 (t, $J=7.10 \mathrm{~Hz}, 3 \mathrm{H}) ;{ }^{13} \mathrm{C}$ NMR (100 MHz, $\left.\mathrm{CDCl}_{3}\right) \delta$ 212.3, 167.2, 141.8, 128.5, 128.4, 125.9, 103.0, 80.9, 68.5, 61.3, 36.8, 32.0, 14.2; IR (film) 
3432, 2253, 1643, 1260, 911, 742, $650 \mathrm{~cm}^{-1}$; HRMS (EI) : m/z calcd. For $\mathrm{C}_{15} \mathrm{H}_{18} \mathrm{O}_{3}$ : 246.1256; found: 228.1150 .

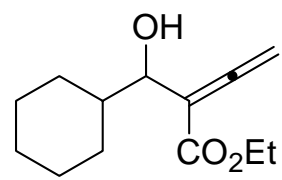

5d

2-(Cyclohexylhydroxymethyl)buta-2,3-dienoic acid ethyl ester (5d): ${ }^{1} \mathrm{H}$ NMR (400 MHz, $\left.\mathrm{CDCl}_{3}\right) \delta 5.23(\mathrm{~s}, 2 \mathrm{H}), 4.23(\mathrm{q}, J=7.11 \mathrm{~Hz}, 2 \mathrm{H}), 4.07(\mathrm{t}, J=7.84 \mathrm{~Hz}, 1 \mathrm{H}), 2.01$ (d, $J=13.00 \mathrm{~Hz}, 1 \mathrm{H}), 1.78-1.74(\mathrm{~m}, 2 \mathrm{H}), 1.70-1.59$ (m, 3H), 1.31 (t, $J=7.11 \mathrm{~Hz}, 3 \mathrm{H})$, 1.25-1.12 (m, 4H), 1.07-0.99 (m, 2H); ${ }^{13} \mathrm{C}$ NMR (100 MHz, $\left.\mathrm{CDCl}_{3}\right) \delta$ 212.9, 167.0, 101.6, 79.9, 75.0, 61.2, 42.6, 29.9, 28.6, 26.4, 26.0, 25.9, 14.2; IR (film) 3431, 3054, 1640, 1421, 1265, 895, $740 \mathrm{~cm}^{-1}$; HRMS (EI) : m/z calcd. For $\mathrm{C}_{13} \mathrm{H}_{20} \mathrm{O}_{3}: 224.1412$; found: 224.1415 .

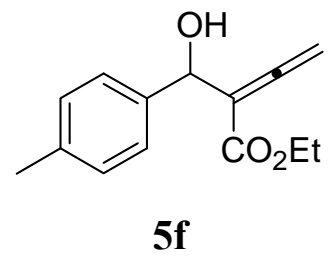

2-(Hydroxy-p-tolylmethyl)buta-2,3-dienoic acid ethyl ester (5f): ${ }^{1} \mathrm{H}$ NMR $(400 \mathrm{MHz}$, $\left.\mathrm{CDCl}_{3}\right) \delta 7.28(\mathrm{~d}, J=8.00 \mathrm{~Hz}, 2 \mathrm{H}), 7.14(\mathrm{~d}, J=8.00 \mathrm{~Hz}, 2 \mathrm{H}), 5.53(\mathrm{~d}, J=6.03 \mathrm{~Hz}, 1 \mathrm{H})$, 5.20 (s, 2H), 4.20 (q, $J=7.14 \mathrm{~Hz}, 2 \mathrm{H}), 3.40$ (d, $J=6.03 \mathrm{~Hz}, 1 \mathrm{H}), 2.33$ (s, 3H), 1.26 (t, $J$ = 7.14 Hz, 2H); ${ }^{13} \mathrm{C}$ NMR (100 MHz, $\left.\mathrm{CDCl}_{3}\right) \delta 213.3,167.1,138.9,137.8,129.3,126.6$, 104.2, 81.4, 72.1, 61.8, 21.6, 14.5; IR (film) 3465, 2919, 1965, 1708, 1368, 1255, 1097, 1038, $747 \mathrm{~cm}^{-1}$; HRMS (EI) : m/z calcd. For $\mathrm{C}_{14} \mathrm{H}_{16} \mathrm{O}_{3}$ : 232.1099; found: 232.1099. 


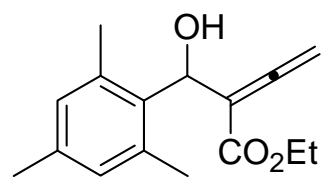

$5 g$

2-[Hydroxy-(2,4,6-trimethylphenyl)methyl]buta-2,3-dienoic acid ethyl ester (5g): ${ }^{1} \mathrm{H}$ NMR (400 MHz, $\mathrm{CDCl}_{3}$ ), $\delta 6.79$ (s, 2H), 6.06-6.04 (m, 1H), 5.06 (dd, $J=3.38 \mathrm{~Hz}, J$ $=3.38 \mathrm{~Hz}, 1 \mathrm{H}), 4.97(\mathrm{dd}, J=3.63 \mathrm{~Hz}, \mathrm{~J}=3.63 \mathrm{~Hz}, 1 \mathrm{H}), 4.29-4.21(\mathrm{~m}, 2 \mathrm{H}), 3.27$ (d, $J=$ $3.00 \mathrm{~Hz}, 1 \mathrm{H}), 2.36$ (s, 6H), 2.23 (s, 3H), 1.30 (t, $J=7.12 \mathrm{~Hz}, 3 \mathrm{H}) ;{ }^{13} \mathrm{C}$ NMR (100 MHz, $\left.\mathrm{CDCl}_{3}\right) \delta 212.3,167.3,136.9,136.9,132.7,129.7,102.4,80.4,68.0,61.4,20.9,20.7$, 14.2; IR (film) 3433, 2252, 1967, 1641, 1370, 1265, 907, 728, $649 \mathrm{~cm}^{-1}$; HRMS (EI) : m/z calcd. For $\mathrm{C}_{16} \mathrm{H}_{20} \mathrm{O}_{3}$ : 260.1412; found: 260.1410 .

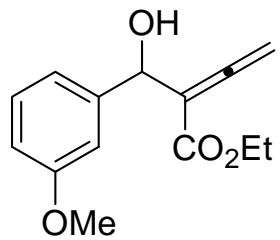

$5 h$

2-[Hydroxy(3-methoxy-phenyl)methyl]buta-2,3-dienoic acid ethyl ester (5h): ${ }^{1} \mathrm{H}$ NMR (400 MHz, $\left.\mathrm{CDCl}_{3}\right) \delta$ 7.26-7.23 (m, 1H), 6.98-6.96 (m, 2H), 6.83-6.80 (m, 1H), 5.55 (d, $J=6.43 \mathrm{~Hz}, 1 \mathrm{H}), 5.20$ (s, 2H), 4.21 (q, $J=7.14 \mathrm{~Hz}, 2 \mathrm{H}), 3.81$ (s, 3H), 3.50 (d, $J=6.43 \mathrm{~Hz}, 1 \mathrm{H}), 1.26(\mathrm{t}, J=7.14 \mathrm{~Hz}, 2 \mathrm{H}) ;{ }^{13} \mathrm{C} \mathrm{NMR}\left(100 \mathrm{MHz}, \mathrm{CDCl}_{3}\right) \delta 213.4,167.2$, 160.0, 143.5, 129.6, 119.0, 113.6, 112.2, 103.9, 81.4, 72.3, 61.8, 55.6, 14.5; IR (film) 3465, 1964, 1704, 1600, 1486, 1254, 1037, 855, $784 \mathrm{~cm}^{-1}$; HRMS (EI) : m/z calcd. For $\mathrm{C}_{14} \mathrm{H}_{16} \mathrm{O}_{4}$ : 248.1049; found: 248.1048 . 


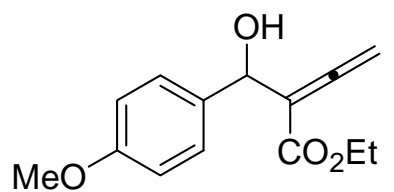

$5 \mathbf{i}$

2-[Hydroxy(4-methoxyphenyl)methyl]buta-2,3-dienoic acid ethyl ester (5i): ${ }^{1} \mathrm{H}$ NMR (400 MHz, $\left.\mathrm{CDCl}_{3}\right) \delta 7.32(\mathrm{~d}, J=8.61 \mathrm{~Hz}, 2 \mathrm{H}), 6.87$ (d, $\left.J=8.61 \mathrm{~Hz}, 2 \mathrm{H}\right), 5.53$ (d, $J=5.95 \mathrm{~Hz}, 1 \mathrm{H}), 5.19$ (s, 2H), 4.21 (q, $J=7.12 \mathrm{~Hz}, 2 \mathrm{H}), 3.80$ (s, 3H), 3.35 (d, $J=5.95$ $\mathrm{Hz}, 1 \mathrm{H}), 1.26$ (t, $J=7.12 \mathrm{~Hz}, 3 \mathrm{H}) ;{ }^{13} \mathrm{C}$ NMR $\left(100 \mathrm{MHz}, \mathrm{CDCl}_{3}\right) \delta$ 212.9, 166.8, 159.1, 133.6, 127.6, 113.6, 104.0, 81.1, 71.5, 61.4, 55.3, 14.1; IR (film) 3431, 3054, 1643, 1422, 1265, $740 \mathrm{~cm}^{-1}$; HRMS (EI) : m/z calcd. For $\mathrm{C}_{14} \mathrm{H}_{16} \mathrm{O}_{4}$ : 248.1049; found: 248.1049.

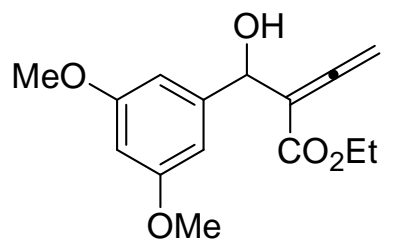

$5 \mathbf{j}$

2-[(3,5-Dimethoxyphenyl)hydroxymethyl]buta-2,3-dienoic acid ethyl ester $(5 \mathbf{j}):{ }^{1} \mathrm{H}$ NMR (400 MHz, $\left.\mathrm{CDCl}_{3}\right) \delta 6.57$ (s, 2H), 6.38 (s, 1H), 5.50 (d, $\left.J=5.93 \mathrm{~Hz}, 1 \mathrm{H}\right), 5.22$ (s, 2H), 4.21 (q, $J=7.10 \mathrm{~Hz}, 2 \mathrm{H}), 3.79(\mathrm{~s}, 6 \mathrm{H}), 3.48$ (d, $J=5.93 \mathrm{~Hz}, 1 \mathrm{H}), 1.27$ (t, $J=7.10$ $\mathrm{Hz}, 3 \mathrm{H}) ;{ }^{13} \mathrm{C} \mathrm{NMR}\left(100 \mathrm{MHz}, \mathrm{CDCl}_{3}\right) \delta 213.0,166.8,160.7,144.0,104.2,103.3,99.7$, 81.0, 72.0, 61.4, 55.4, 29.7, 14.2; IR (film) 3466, 2921, 2849, 1707, 1597, 1459, 1262, 1204, 1156, 909, $732 \mathrm{~cm}^{-1}$; HRMS (EI) : m/z calcd. For $\mathrm{C}_{15} \mathrm{H}_{18} \mathrm{O}_{5}$ : 278.1154; found: 278.1155 . 


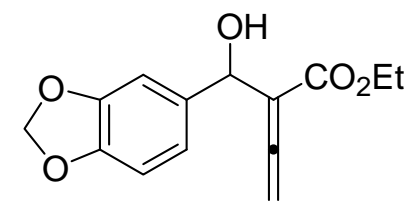

5k

2-(Benzo[1,3]dioxol-5-yl-hydroxymethyl)buta-2,3-dienoic acid ethyl ester $(5 \mathrm{k})$ : ${ }^{1} \mathrm{H}$ NMR (400 MHz, $\left.\mathrm{CDCl}_{3}\right) \delta 6.90(\mathrm{~s}, 1 \mathrm{H}), 6.86(\mathrm{~d}, J=8.03 \mathrm{~Hz}, 1 \mathrm{H}), 6.76(\mathrm{~d}, J=8.03 \mathrm{~Hz}$, 1H), 5.94 (s, 2H), 5.48 (d, $J=5.68 \mathrm{~Hz}, 1 \mathrm{H}), 5.21$ (s, 2H), 4.20 (q, $J=7.16 \mathrm{~Hz}, 2 \mathrm{H})$, 3.45 (s, 1H), 1.26 (t, $J=7.16 \mathrm{~Hz}, 3 \mathrm{H}) ;{ }^{13} \mathrm{C}$ NMR (100 MHz, $\left.\mathrm{CDCl}_{3}\right) \delta 212.8,166.7$, 147.5, 147.0, 135. 5, 119.8, 107.9, 107.0, 103.9, 101.0, 81.2, 71.5, 61.4, 14.1; IR (film) 3476, 2985, 2901, 1965, 1705, 1487, 1443, 1249, 1038, $928 \mathrm{~cm}^{-1}$; HRMS (EI) : m/z calcd. For $\mathrm{C}_{14} \mathrm{H}_{14} \mathrm{O}_{5}$ : 262.0841; found: 262.0842 .<smiles>C=C(C(=O)OCC)C(O)c1cc(OC)c(OC)c(OC)c1</smiles>

51

2-[Hydroxy(3,4,5-trimethoxyphenyl)methyl]buta-2,3-dienoic acid ethyl ester (5l): ${ }^{1} \mathrm{H}$ NMR (400 MHz, $\mathrm{CDCl}_{3}$ ) $\delta 6.64$ (s, 2H), 5.52 (d, $\left.J=5.23 \mathrm{~Hz}, 1 \mathrm{H}\right), 5.21$ (s, 2H), 4.23 (q, $J=7.14 \mathrm{~Hz}, 2 \mathrm{H}), 3.52$ (d, $J=5.23 \mathrm{~Hz}, 1 \mathrm{H}), 1.28(\mathrm{t}, J=7.14 \mathrm{~Hz}, 2 \mathrm{H}) ;{ }^{13} \mathrm{C} \mathrm{NMR}$ $\left(100 \mathrm{MHz}, \mathrm{CDCl}_{3}\right) \delta 213.3,167.2,153.5,137.8,137.5,104.1,103.7,81.6,72.3,61.9$, 61.2, 56.5, 14.6; IR (film) 3484, 2938, 1964, 1708, 1592, 1506, 1461, 1420, 1328, 1251, 1127, 1053, $854 \mathrm{~cm}^{-1}$; HRMS (EI) : m/z calcd. For $\mathrm{C}_{16} \mathrm{H}_{20} \mathrm{O}_{6}$ : 308.1260; found: 308.1263. 


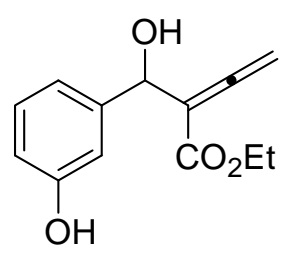

$5 \mathrm{~m}$

2-[Hydroxy-(3-hydroxyphenyl)methyl]buta-2,3-dienoic acid ethyl ester (5m): ${ }^{1} \mathrm{H}$ NMR (400 MHz, $\left.\mathrm{CDCl}_{3}\right) \delta 7.16(\mathrm{t}, J=7.87 \mathrm{~Hz}, 1 \mathrm{H}), 7.07$ (d, $\left.J=7.87 \mathrm{~Hz}, 1 \mathrm{H}\right), 6.91-$ 6.87 (m, 2H), 6.51 (s, 1H), 5.52 (s, 1H), 5.19 (s, 2H), 4.18 (q, $J=7.13$ Hz, 2H), 3.88 (bs, 1H), 1.26 (t, $J=7.13 \mathrm{~Hz}, 3 \mathrm{H}) ;{ }^{13} \mathrm{C}$ NMR (100 MHz, $\left.\mathrm{CDCl}_{3}\right) \delta 213.5,167.3,156.3$, 143.4, 129.9, 118.8, 115.3, 113.8, 103.9, 81.8, 72.0, 62.0, 14.5; IR (film) 3432, 2253, 1967, 1641, 1456, 1370, 1265, 907, 728, $649 \mathrm{~cm}^{-1}$; HRMS (EI) : m/z calcd. For $\mathrm{C}_{13} \mathrm{H}_{14} \mathrm{O}_{4}$ : 234.0892; found: 234.0891.<smiles>C=C(C)C(O)C(O)c1cc(O)cc(O)c1</smiles>

5n

2-[(3,5-Dihydroxyphenyl)hydroxymethyl]buta-2,3-dienoic acid ethyl ester $(5 n):{ }^{1} \mathrm{H}$ NMR (400 MHz, DMSO), $\delta 9.09$ (s, 2H), 6.21 (s, 2H), 6.04 (s, 1H), 5.51 (d, $J=4.77$ Hz, 1H), 5.41 (dd, $J=2.10 \mathrm{~Hz}, J=2.10 \mathrm{~Hz}, 1 \mathrm{H}), 5.34$ (dd, $J=1.85 \mathrm{~Hz}, J=1.85 \mathrm{~Hz}$, 1H), 5.24 (s, 1H), 4.13-4.06 (m, 2H), 1.16 (t, $J=7.10 \mathrm{~Hz}, 3 \mathrm{H}) ;{ }^{13} \mathrm{C}$ NMR (100 MHz, DMSO) $\delta$ 213.2, 165.8, 158.3, 145.9, 105.5, 104.9, 101.6, 82.1, 69.4, 60.8, 14.5; IR (film) 3292, 2921, 1964, 1703, 1604, 1455, 1259, 999, $852 \mathrm{~cm}^{-1}$; HRMS (EI) : m/z calcd. For $\mathrm{C}_{13} \mathrm{H}_{14} \mathrm{O}_{5}$ : 250.0841; found: 250.0839 . 


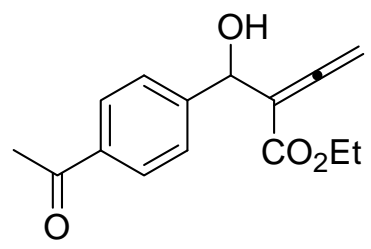

50

2-[(4-Acetylphenyl)hydroxymethyl]buta-2,3-dienoic acid ethyl ester (5o): ${ }^{1} \mathrm{H}$ NMR (400 MHz, $\mathrm{CDCl}_{3}$ ), $\delta 7.94$ (d, $\left.J=8.30 \mathrm{~Hz}, 2 \mathrm{H}\right), 7.50$ (d, $\left.J=8.30 \mathrm{~Hz}, 2 \mathrm{H}\right), 5.63$ (s, 1H), 5.19 (s, 2H), 4.22 (q, $J=7.11 \mathrm{~Hz}, 2 \mathrm{H}), 3.66$ (s, 1H), 2.60 (s, 3H), 1.27 (t, $J=7.11 \mathrm{~Hz}$, 3H); ${ }^{13} \mathrm{C}$ NMR (100 MHz, $\left.\mathrm{CDCl}_{3}\right) \delta 213.0,197.9,166.7,146.6,136.4,128.3,126.4$, 103.2, 81.3, 71.6, 61.6, 26.7, 14.1; IR (film) 3419, 2917, 1965, 1678, 1608, 1265, 736 $\mathrm{cm}^{-1}$; HRMS (EI) : m/z calcd. For $\mathrm{C}_{15} \mathrm{H}_{16} \mathrm{O}_{4}$ : 260.1049; found: 260.1051 .

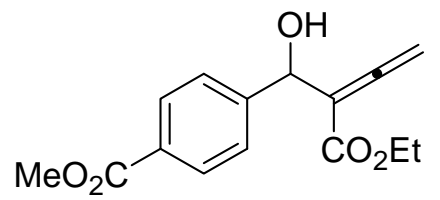

$5 p$

4-(2-Ethoxycarbonyl-1-hydroxy-buta-2,3-dienyl)benzoic acid methyl ester (5p): ${ }^{1} \mathrm{H}$ NMR (400 MHz, $\mathrm{CDCl}_{3}$ ), $\delta 8.01$ (d, $\left.J=8.39 \mathrm{~Hz}, 2 \mathrm{H}\right), 7.47$ (d, $\left.J=8.39 \mathrm{~Hz}, 2 \mathrm{H}\right), 5.63$ (d, $J=5.61 \mathrm{~Hz}, 1 \mathrm{H}), 5.17$ (s, 2H), 4.21 (q, $J=7.14 \mathrm{~Hz}, 2 \mathrm{H}), 3.91(\mathrm{~s}, 3 \mathrm{H}), 3.63$ (d, $J=5.61$ $\mathrm{Hz}, 1 \mathrm{H}), 1.27$ (t, $J=7.14 \mathrm{~Hz}, 3 \mathrm{H}) ;{ }^{13} \mathrm{C}$ NMR $\left(100 \mathrm{MHz}, \mathrm{CDCl}_{3}\right) \delta 213.0,166.9,166.7$, 146.4, 129.5, 129.5, 126.2, 103.3, 81.3, 71.6, 61.6, 52.1, 14.1; IR (film) 3475, 2918, 1965, 1709, 1279, 1109, $734 \mathrm{~cm}^{-1}$; HRMS (EI) : m/z calcd. For $\mathrm{C}_{15} \mathrm{H}_{16} \mathrm{O}_{5}: 276.0998$; found: 276.0998.<smiles>C=CC(=O)C(O)C(O)c1ccc([N+](=O)[O-])cc1</smiles>

$5 q$ 
2-[Hydroxy-(4-nitrophenyl)methyl]buta-2,3-dienoic acid ethyl ester (5q): ${ }^{1} \mathrm{H}$ NMR (400 MHz, $\mathrm{CDCl}_{3}$ ), $\delta 8.20$ (d, $\left.J=8.74 \mathrm{~Hz}, 2 \mathrm{H}\right), 7.58$ (d, $\left.J=8.74 \mathrm{~Hz}, 2 \mathrm{H}\right), 5.67$ (bs, 1H), 5.20 (s, 2H), 4.23 (q, $J=7.10 \mathrm{~Hz}, 2 \mathrm{H}), 3.74$ (d, $J=5.62 \mathrm{~Hz}, 1 \mathrm{H}), 1.28$ (t, $J=7.10$ $\mathrm{Hz}, 3 \mathrm{H}) ;{ }^{13} \mathrm{C}$ NMR $\left(100 \mathrm{MHz}, \mathrm{CDCl}_{3}\right) \delta 213.3,167.0,148.9,147.8,127.5,123.8,103.4$, 82.0, 71.6, 62.2, 14.5; IR (film) 3465, 2917, 1966, 1704, 1606, 1348, 1266, 1042, 736 $\mathrm{cm}^{-1}$; HRMS (EI) : m/z calcd. For $\mathrm{C}_{13} \mathrm{H}_{13} \mathrm{NO}_{5}$ : 263.0794; found: 263.0796.

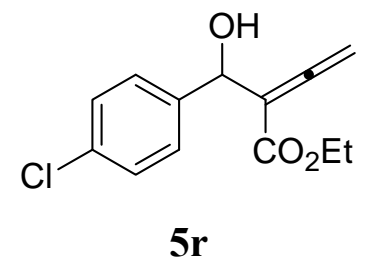

2-[(4-Chlorophenyl)hydroxymethyl]buta-2,3-dienoic acid ethyl ester (5r): ${ }^{1} \mathrm{H}$ NMR (400 MHz, $\mathrm{CDCl}_{3}$ ) $\delta$ 7.35-7.29 (m, 4H), 5.55 (d, $\left.J=5.94 \mathrm{~Hz}, 1 \mathrm{H}\right), 5.18$ (s, 2H), 4.22 (q, $J=7.13 \mathrm{~Hz}, 2 \mathrm{H}), 3.51$ (d, $J=5.94 \mathrm{~Hz}, 1 \mathrm{H}), 1.27$ (t, $J=7.13 \mathrm{~Hz}, 3 \mathrm{H}) ;{ }^{13} \mathrm{C} \mathrm{NMR}$ $\left(100 \mathrm{MHz}, \mathrm{CDCl}_{3}\right) \delta 213.3,167.1,140.2,133.8,128.8,128.1,103.9,81.6,71.8,61.9$, 14.5; IR (film) 3433, 2918, 2849, 1966, 1704, 1643, 1265, 1091, $738 \mathrm{~cm}^{-1}$; HRMS (EI) : m/z calcd. For $\mathrm{C}_{13} \mathrm{H}_{13} \mathrm{ClO}_{3}$ : 252.0553; found: 252.0554 .<smiles>C=CC(C(=O)OCC)=C(O)c1ccccc1I</smiles>

5s

2-[Hydroxy(2-iodophenyl)methyl]buta-2,3-dienoic acid ethyl ester (5s): ${ }^{1} \mathrm{H}$ NMR $\left(400 \mathrm{MHz}, \mathrm{CDCl}_{3}\right) \delta 7.79$ (d, $\left.J=7.95 \mathrm{~Hz}, 1 \mathrm{H}\right), 7.59$ (d, $\left.J=7.86 \mathrm{~Hz}, 1 \mathrm{H}\right), 7.37$ (t, $J=$ $7.95 \mathrm{~Hz}, 1 \mathrm{H}$ ), 7.00-6.96 (m, 1H), 5.79-5.77 (m, 1H), 5.02 (dd, $J=2.43 \mathrm{~Hz}, J=2.43 \mathrm{~Hz}$, 1H), 4.95 (dd, $J=2.19 \mathrm{~Hz}, J=2.19 \mathrm{~Hz}, 1 \mathrm{H}), 4.31-4.23$ (m, 2H), 3.61 (d, $J=3.87 \mathrm{~Hz}$, 
1H), $1.31(\mathrm{t}, J=7.13 \mathrm{~Hz}, 3 \mathrm{H}) ;{ }^{13} \mathrm{C}$ NMR (100 MHz, $\left.\mathrm{CDCl}_{3}\right) \delta 213.1,167.1,142.6$, 139.0, 129.3, 128.2, 127.9, 102.9, 98.0, 81.0, 75.1, 61.6, 14.2; IR (film) 3433, 3054, 2987, 1967, 1641, 1421, 1265, $742 \mathrm{~cm}^{-1}$; HRMS (EI) : m/z calcd. For $\mathrm{C}_{13} \mathrm{H}_{13} \mathrm{IO}_{3}$ : 343.9909; found: 343.9907.

Typical experimental procedure for gold-catalyzed cyclization of 2-[hydroxy-(3hydroxyphenyl)methyl]buta-2,3-dienoic acid ethyl ester to hydroxynaphthalene-2carboxylic acid ethyl ester: To solution of $5 \mathrm{~mol} \%$ of chlorotriphenylphosphinegold (12.4 mg, $0.025 \mathrm{mmol}$ ) and $5 \mathrm{~mol} \%$ silver trifluoromethanesulfonate (6.4 mg, 0.025 mmol) in dry dichloromethane $(2.5 \mathrm{~mL})$ under a nitrogen atmosphere was added 2[hydroxy-(3-hydroxyphenyl)methyl]buta-2,3-dienoic acid ethyl ester (117.1 mg, 0.5 mmol). The reaction mixture was stirred at room temperature. After $1 \mathrm{~h}$, reaction mixture was filtered and then, concentrated in vacuo. The residue was subjected to flash column chromatography on silica gel (ethyl acetate:hexane $=1: 5$ ) to give 5-hydroxynaphthalene-2-carboxylic acid ethyl ester 6e (19.5 mg, 18\%), 7-hydroxynaphthalene-2carboxylic acid ethyl ester 6 f (62.7 mg, 58\%) and 2-(3-hydroxyphenyl)-3-ethoxycarbonyl-2,5-dihydrofuran ( $24.6 \mathrm{mg}, 21 \%$ ).

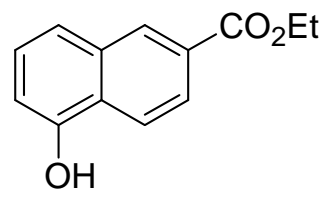

6e

5-Hydroxynaphthalene-2-carboxylic acid ethyl ester (6e): pale yellow solid; mp = 141-143 ${ }^{\circ} \mathrm{C} ;{ }^{1} \mathrm{H}$ NMR (400 MHz, $\mathrm{CDCl}_{3}$ ) $\delta 8.58$ (s, $\left.1 \mathrm{H}\right), 8.26$ (d, $\left.J=8.81 \mathrm{~Hz}, 1 \mathrm{H}\right), 8.07$ (d, $J=8.81 \mathrm{~Hz}, 1 \mathrm{H}), 7.54$ (d, $J=7.90 \mathrm{~Hz}, 1 \mathrm{H}), 7.37$ (t, $J=7.90 \mathrm{~Hz}, 1 \mathrm{H}), 6.95$ (d, $J=$ 
$7.90 \mathrm{~Hz}, 1 \mathrm{H}), 5.96$ (s, 1H), 4.46 (q, $J=7.11 \mathrm{~Hz}, 2 \mathrm{H}), 1.46$ (t, $J=7.11 \mathrm{~Hz}, 2 \mathrm{H}) ;{ }^{13} \mathrm{C}$ NMR (100 MHz, $\left.\mathrm{CDCl}_{3}\right) \delta 167.5,152.0,134.3,131.1,128.5,127.1,126.9,124.9,122.6$, 122.3, 111.3, 61.7, 14.8; IR (film) 3366, 1692, 1630, 1581, 1473, 1378, 1291, 1021, 773 $\mathrm{cm}^{-1}$; HRMS (EI) : m/z calcd. For $\mathrm{C}_{13} \mathrm{H}_{12} \mathrm{O}_{3}$ : 216.0786; found: 216.0783.

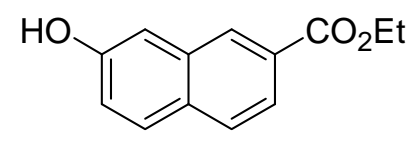

6f

7-Hydroxynaphthalene-2-carboxylic acid ethyl ester $(6)^{2}$ : pale yellow solid; $\mathrm{mp}=$ 148-149 ${ }^{\circ} \mathrm{C} ; \quad{ }^{1} \mathrm{H}$ NMR (400 MHz, $\left.\mathrm{CDCl}_{3}\right) \delta 8.38$ (s, 1H), 7.85 (d, $\left.J=8.54 \mathrm{~Hz}, 1 \mathrm{H}\right)$, 7.75-7.71 (m, 2H), 7.21 (d, $J=2.42 \mathrm{~Hz}, 1 \mathrm{H}), 7.15$ (d, $J=8.79 \mathrm{~Hz}, 1 \mathrm{H}), 5.25$ (bs, 1H), 4.37 (q, $J=7.10 \mathrm{~Hz}, 2 \mathrm{H}), 1.38(\mathrm{t}, J=7.10 \mathrm{~Hz}, 2 \mathrm{H}) ;{ }^{13} \mathrm{C} \mathrm{NMR}\left(100 \mathrm{MHz}, \mathrm{CDCl}_{3}\right) \delta$ 167.4, 154.4, 134.1, 131.4, 130.1, 129.8, 128.7, 128.7, 128.4, 123.5, 120.6, 111.1, 61.6, 14.8; IR (film) 3398, 1689, 1629, 1608, 1444, 1360, 1299, 1281, 1231, 1206, 845, 650 $\mathrm{cm}^{-1}$.

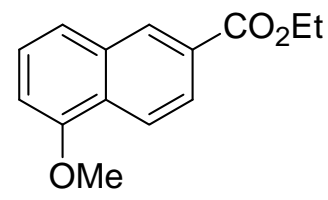

6a

5-Methoxynaphthalene-2-carboxylic acid ethyl ester (6a): pale yellow oil; ${ }^{1} \mathrm{H}$ NMR (400 MHz, $\left.\mathrm{CDCl}_{3}\right) \delta 8.56(\mathrm{~s}, 1 \mathrm{H}), 8.30(\mathrm{~d}, J=8.84 \mathrm{~Hz}, 1 \mathrm{H}), 8.05(\mathrm{~d}, J=8.84 \mathrm{~Hz}, 1 \mathrm{H})$, 7.54 (d, $J=8.02 \mathrm{~Hz}, 1 \mathrm{H}), 7.45$ (t, $J=8.02 \mathrm{~Hz}, 1 \mathrm{H}), 6.92$ (d, $J=8.02 \mathrm{~Hz}, 1 \mathrm{H}), 4.44$ (q, $J$ $=7.14 \mathrm{~Hz}, 2 \mathrm{H}), 4.02(\mathrm{~s}, 3 \mathrm{H}), 1.45(\mathrm{t}, J=7.14 \mathrm{~Hz}, 3 \mathrm{H}) ;{ }^{13} \mathrm{C} \mathrm{NMR}\left(100 \mathrm{MHz}, \mathrm{CDCl}_{3}\right) \delta$ 166.9, 155.3, 133.6, 130.4, 128.2, 127.6, 126.7, 124.6, 122.4, 121.4, 105.9, 61.1, 55.6, 
14.4; IR (film) 3583, 2934, 1715, 1580, 1465, 1368, 1269, 1235, 1203, 1184, 1108, 772 $\mathrm{cm}^{-1}$; HRMS (EI) : m/z calcd. For $\mathrm{C}_{14} \mathrm{H}_{14} \mathrm{O}_{3}$ : 230.0943; found: 230.0945 .

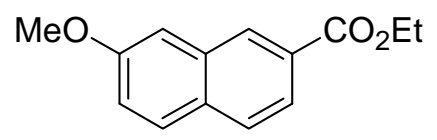

6b

7-Methoxynaphthalene-2-carboxylic acid ethyl ester $(6 b)^{3}$ : pale yellow oil; ${ }^{1} \mathrm{H}$ NMR $\left(400 \mathrm{MHz}, \mathrm{CDCl}_{3}\right) \delta 8.50$ (s, 1H), 7.92 (d, $\left.J=8.55 \mathrm{~Hz}, 1 \mathrm{H}\right), 7.77$ (dd, $J=8.55 \mathrm{~Hz}, J=$ $8.55 \mathrm{~Hz}, 2 \mathrm{H}), 7.25-7.22$ (m, 2H), 4.43 (q, $J=7.10 \mathrm{~Hz}, 2 \mathrm{H}), 3.92$ (s, 3H), 1.44 (t, $J=$ 7.10 Hz, 3H); ${ }^{13} \mathrm{C}$ NMR (100 MHz, $\left.\mathrm{CDCl}_{3}\right) \delta$ 166.9, 158.1, 133.8, 131.1, 129.6, 129.2, 128.2, 127.8, 123.2, 121.1, 106.8, 61.0, 55.3, 14.4; IR (film) 2980, 1715, 1632, 1606, 1513, 1463, 1392, 1282, 1240, 1216, 1172, 1098, 954, 911, 846, $746 \mathrm{~cm}^{-1}$.

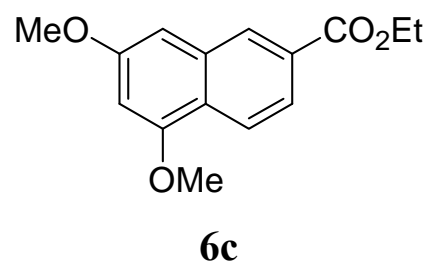

5,7-Dimethoxynaphthalene-2-carboxylic acid ethyl ester (6c): white solid; $\mathrm{mp}=53$ $55{ }^{\circ} \mathrm{C} ;{ }^{1} \mathrm{H}$ NMR (400 MHz, $\left.\mathrm{CDCl}_{3}\right) \delta 8.43$ (s, 1H), 8.17 (d, $\left.J=8.70 \mathrm{~Hz}, 1 \mathrm{H}\right), 7.90$ (d, $J$ $=8.70 \mathrm{~Hz}, 1 \mathrm{H}), 6.82(\mathrm{~s}, 1 \mathrm{H}), 6.57$ (s, 1H), 4.43 (q, $J=7.16 \mathrm{~Hz}, 2 \mathrm{H}), 3.97$ (s, 3H), 3.92 (s, 3H), 1.44 (t, $J=7.16 \mathrm{~Hz}, 3 \mathrm{H}) ;{ }^{13} \mathrm{C}$ NMR (100 MHz, $\left.\mathrm{CDCl}_{3}\right) \delta 167.4,159.1,156.8$, 134.7, 129.6, 129.2, 124.3, 122.9, 122.7, 100.1, 99. 3, 61.4, 56.1, 55.8, 14.8; IR (film) 2938, 1715, 1631, 1604, 1510, 1454, 1337, 1263, 1212, 1189, 1152, 1113, 833, $780 \mathrm{~cm}^{-}$ ; HRMS (EI) : m/z calcd. For $\mathrm{C}_{15} \mathrm{H}_{16} \mathrm{O}_{4}$ : 260.1049; found: 260.1051. 


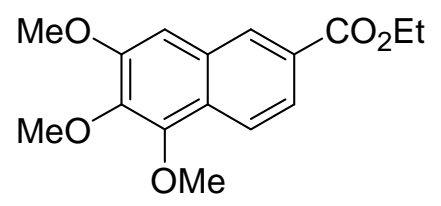

6d

5,6,7-Trimethoxynaphthalene-2-carboxylic acid ethyl ester $(\mathbf{6 d})^{4}$ : yellow solid; mp = 97-99 ${ }^{\circ} \mathrm{C} ;{ }^{1} \mathrm{H}$ NMR $\left(400 \mathrm{MHz}, \mathrm{CDCl}_{3}\right) \delta 8.45$ (s, 1H), 8.07 (d, $\left.J=8.74 \mathrm{~Hz}, 1 \mathrm{H}\right), 7.94$ (d, $J=8.74 \mathrm{~Hz}, 1 \mathrm{H}), 7.05$ (s, 1H), 4.42 (q, $J=7.15 \mathrm{~Hz}, 2 \mathrm{H}), 4.05$ (s, 3H), 4.00 (s, 3H), 3.98 (s, 3H), 1.43 (t, $J=7.15 \mathrm{~Hz}, 3 \mathrm{H}) ;{ }^{13} \mathrm{C}$ NMR (100 MHz, $\left.\mathrm{CDCl}_{3}\right) \delta$ 167.3, 153.9, 148.1, 143.1, 130.3, 129.7, 128.0, 127.2, 123.7, 122.3, 103.9, 61.9, 61.6, 61.4, 56.3, 14.8; IR (film) 2938, 1715, 1603, 1478, 1407, 1330, 1249, 1192, 1107, 1037, 999, 910, $755 \mathrm{~cm}^{-1}$.<smiles>CCOC(=O)c1ccc2c(O)cc(O)cc2c1</smiles>

$6 \mathrm{~g}$

5,7-Dihydroxynaphthalene-2-carboxylic acid ethyl ester (6g): pale orange solid; mp $=174-176{ }^{\circ} \mathrm{C} ;{ }^{1} \mathrm{H}$ NMR (400 MHz, DMSO) $\delta 10.30$ (s, 1H), 9.74 (s, 1H), 8.25 (s, 1H), 8.04 (d, $J=8.73 \mathrm{~Hz}, 1 \mathrm{H}), 7.65$ (d, $J=8.73 \mathrm{~Hz}, 1 \mathrm{H}), 6.77$ (s, 1H), 6.64 (s, 1H), 4.34 (q, $J=7.11 \mathrm{~Hz}, 2 \mathrm{H}), 1.35$ (t, $J=7.11 \mathrm{~Hz}, 2 \mathrm{H}) ;{ }^{13} \mathrm{C}$ NMR (100 MHz, DMSO) $\delta 166.5,157.1$, 154.7, 135.0, 128.7, 128.0, 123.0, 121.9, 120.5, 103.5, 101.8, 61.0, 14.6; IR (film) 3336, 1687, 1633, 1604, 1586, 1524, 1474, 1404, 1369, 1267, 1167, 1143, 1105, 1022, 997, 836, 780, $746 \mathrm{~cm}^{-1}$; HRMS (EI) : m/z calcd. For $\mathrm{C}_{13} \mathrm{H}_{12} \mathrm{O}_{4}$ : 232.0736; found: 232.0734. 


\section{Reference}

(1) Sadao, T.; Hirofumi, K.; Satoshi, T.; Takashi, F.; Takashi, F.; Masanori, U.; J. Org. Chem. 1993, 58, 5952.

(2) James, A. N.; Lawrence, F.; Barbara, J. PCT Int. Appl. 2002, 449 pp.

(3) (a) Mukerjee, Y. N.; Gaur, S. P.; Jain, P. C.; Anand, N. Indian J. Chem., Sect. B, 1985, 24B, 985. (b) Yuho, T.; Masami, S.; Takahiro, F.; Yasuhide, Y. Bull. Chem. Soc. Jpn. 1979, 52, 3033.

(4) Alex, K.; Gottfried, C. Ger. Offen. 1978, 70 pp. 


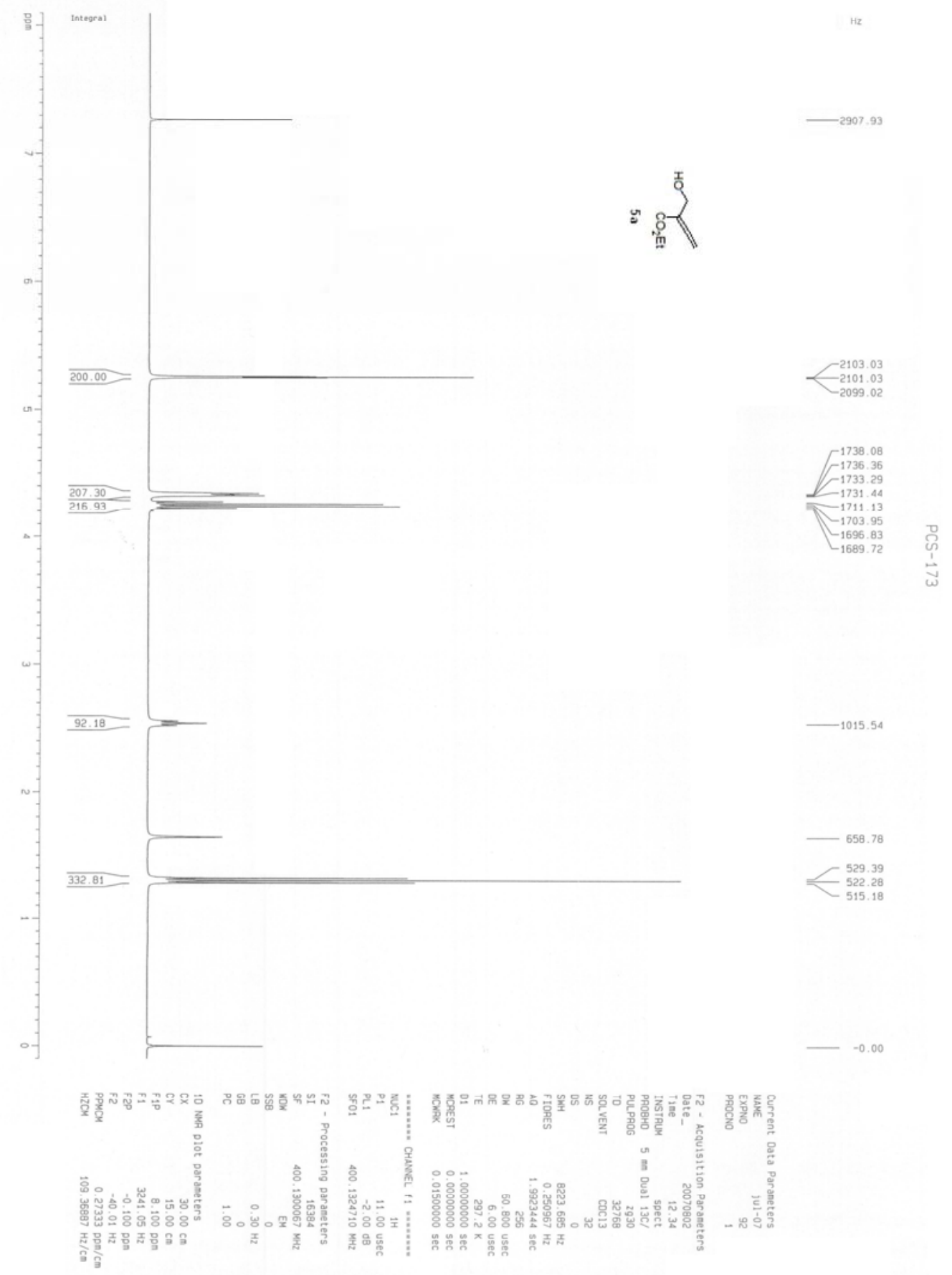




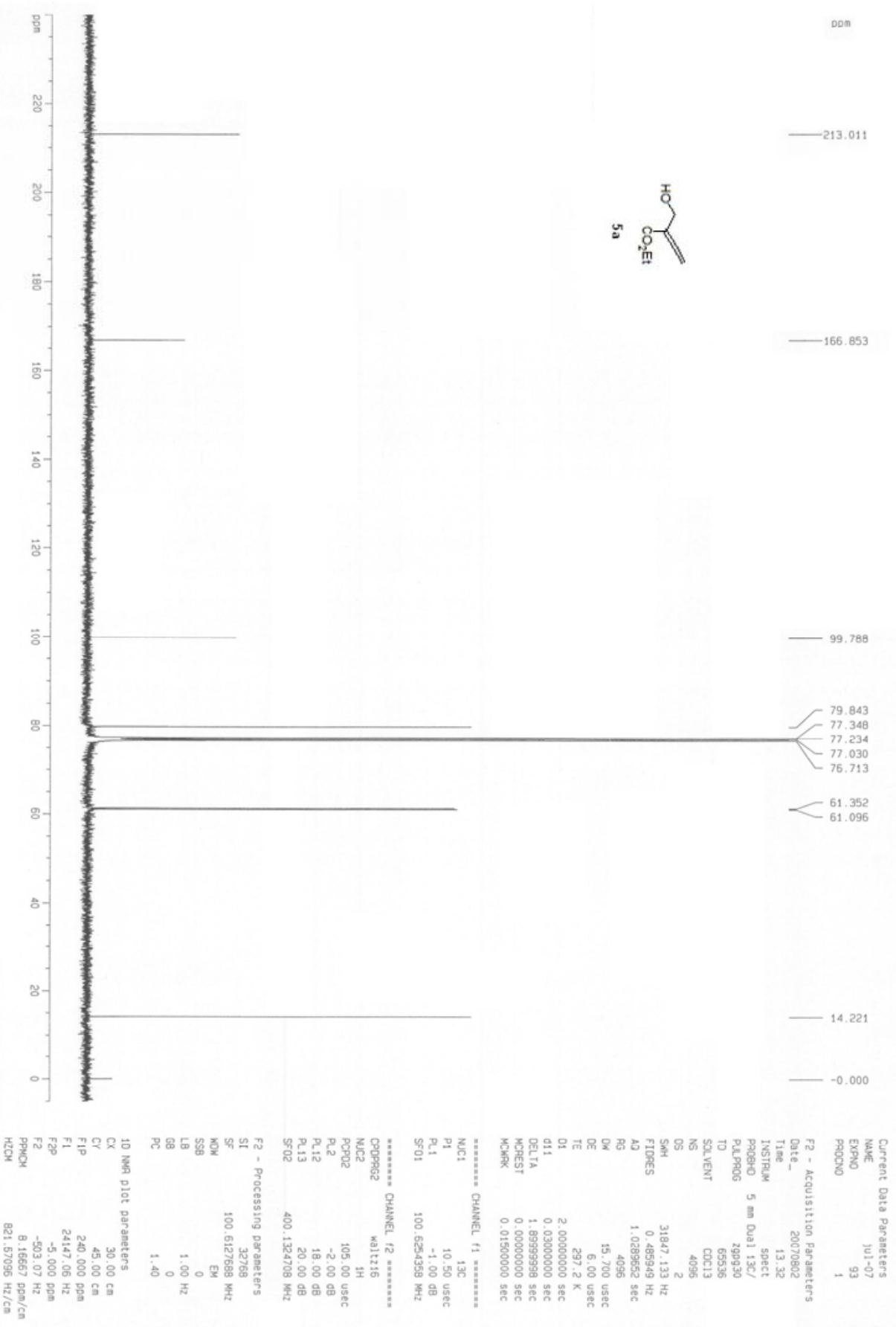




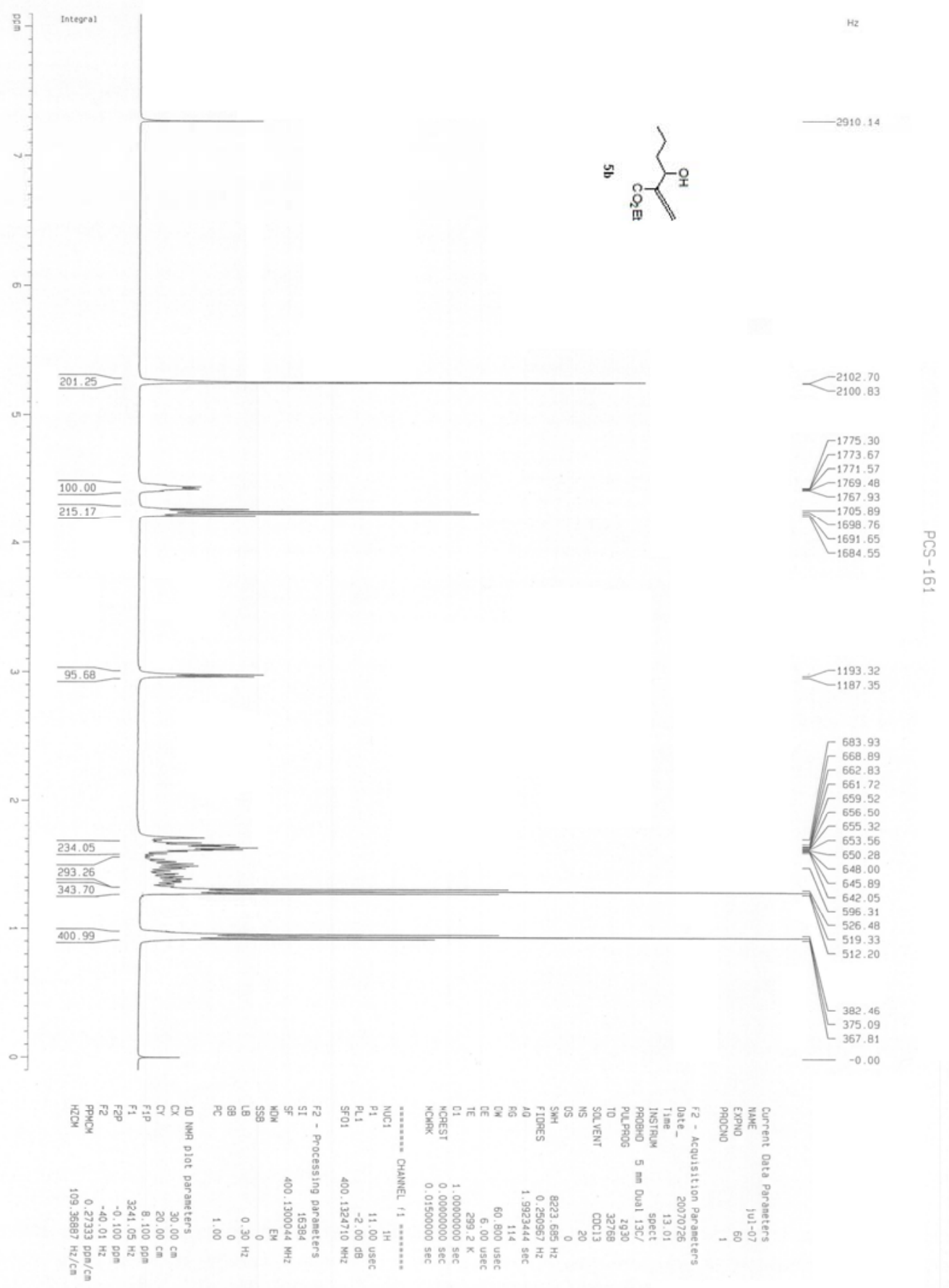



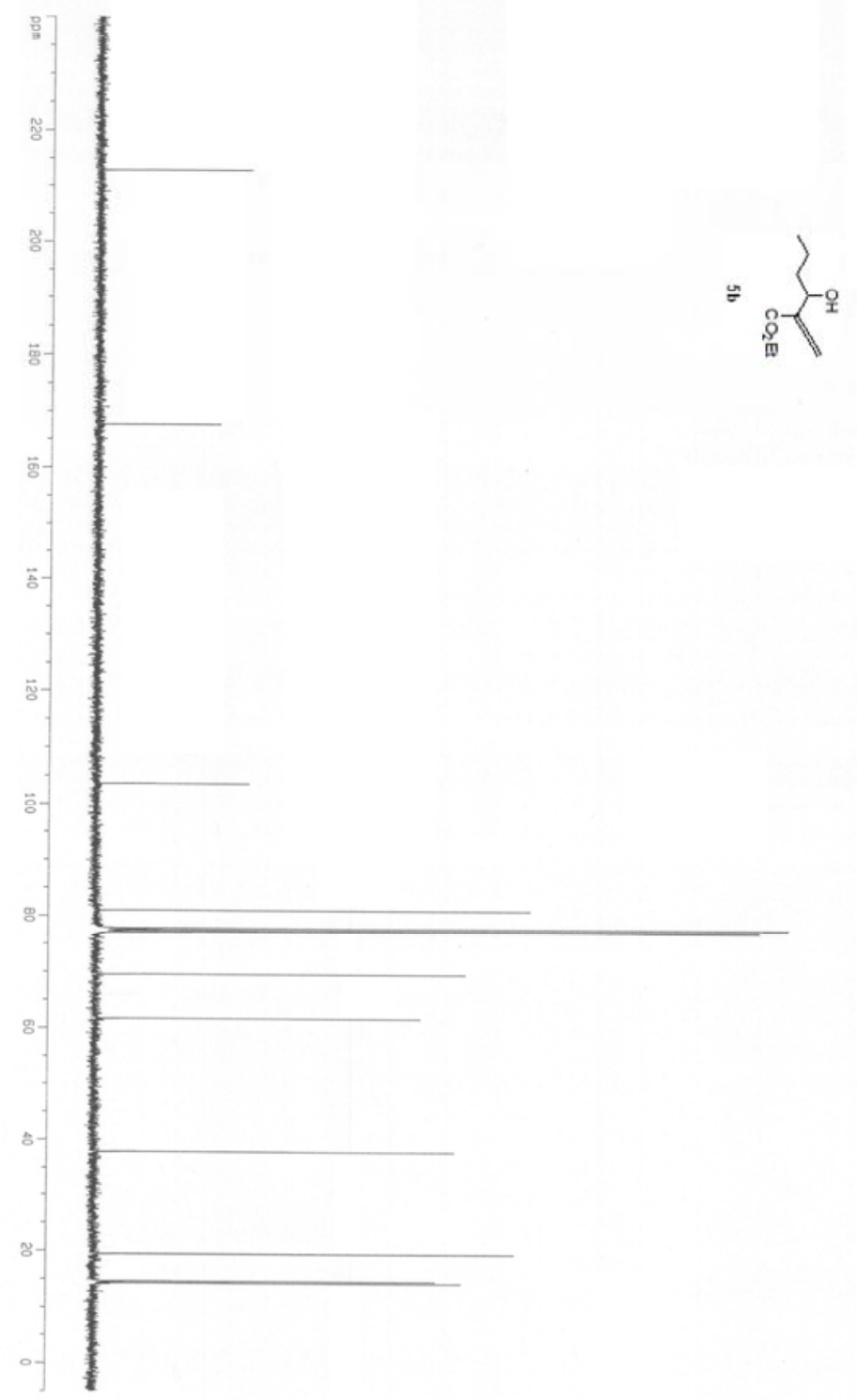

$-167.570$

$-103.555$

\subsection{1
$\quad 77.745$
-73.27}

$\begin{array}{r}77.109 \\ -69.502 \\ \hline\end{array}$

- 61.633

$-37.815$

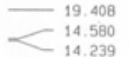

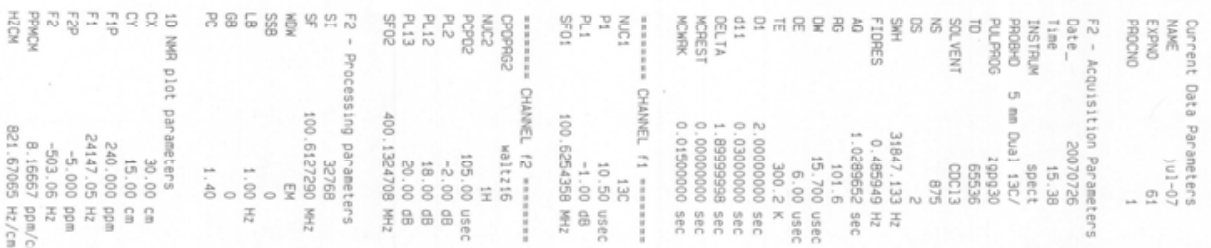




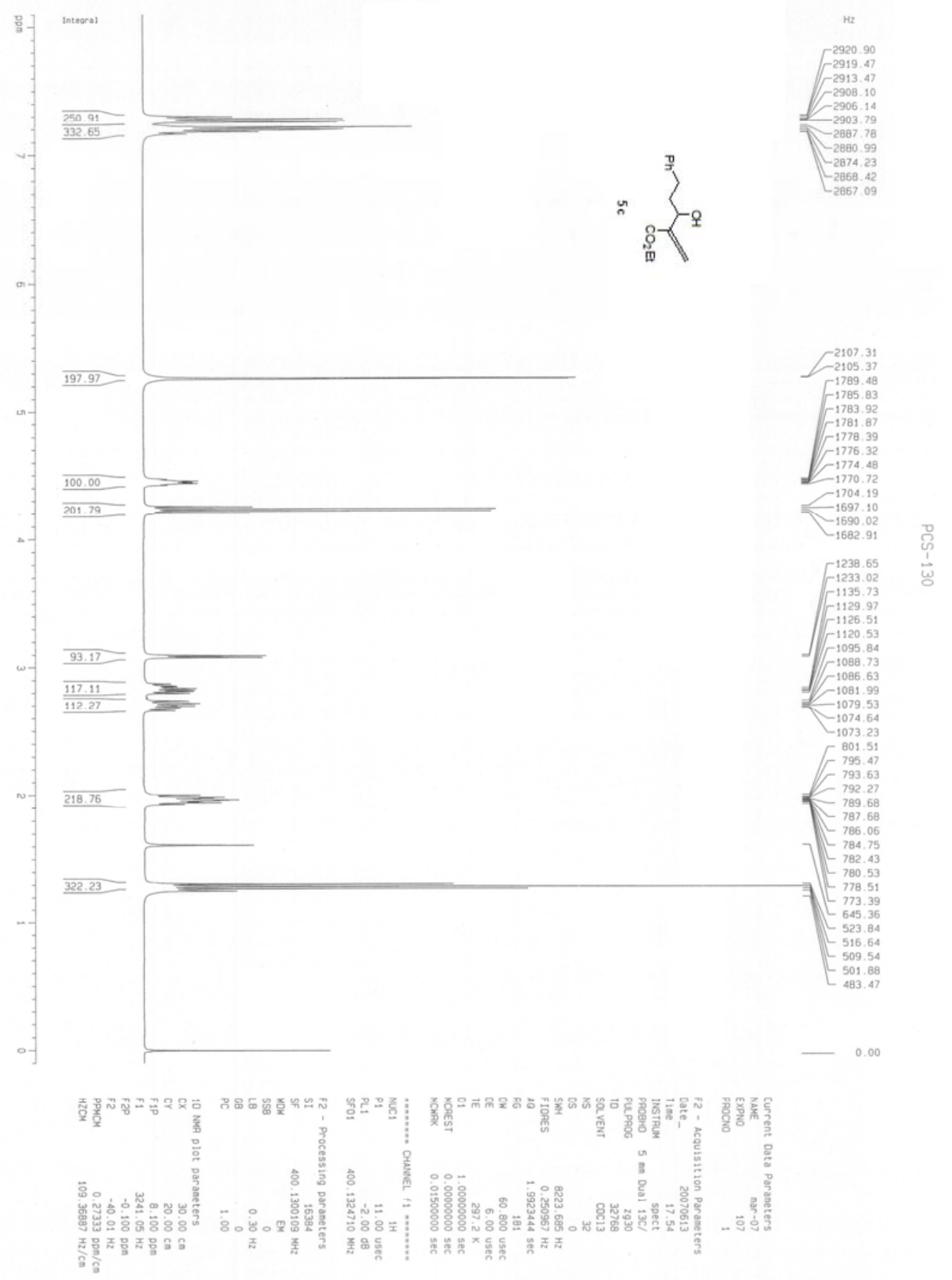



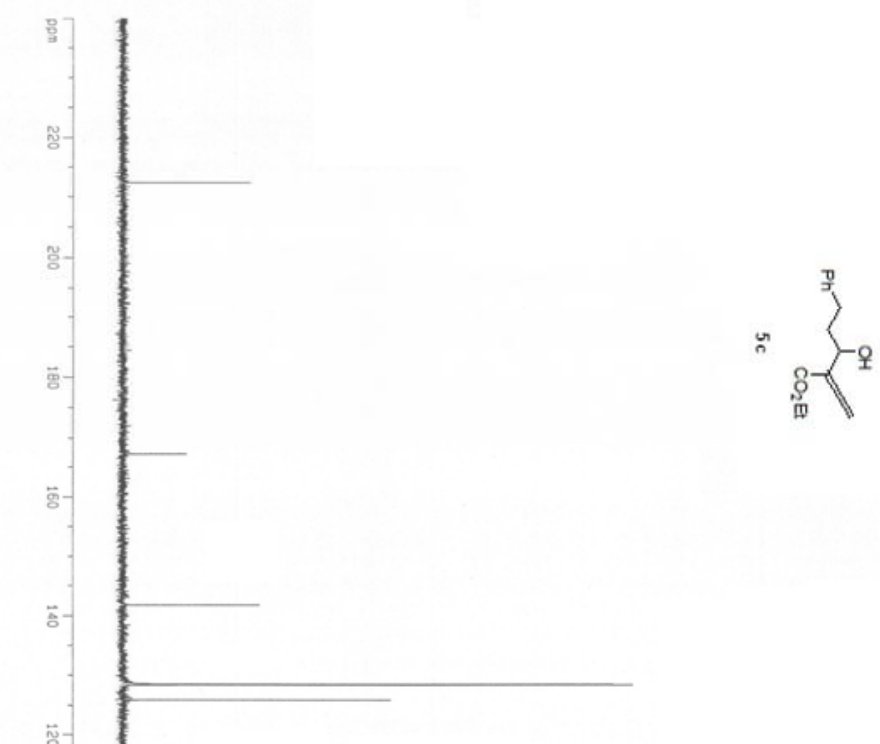

$\begin{array}{r}141.777 \\ r_{128.504}^{128.379} \\ \hline\end{array}$
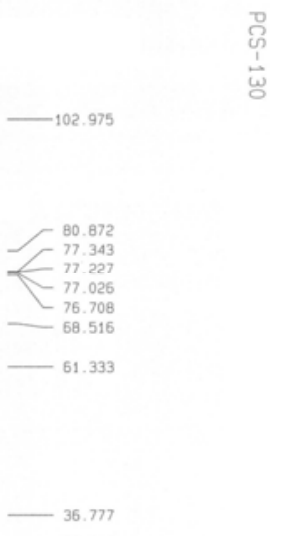

- 14.191

다

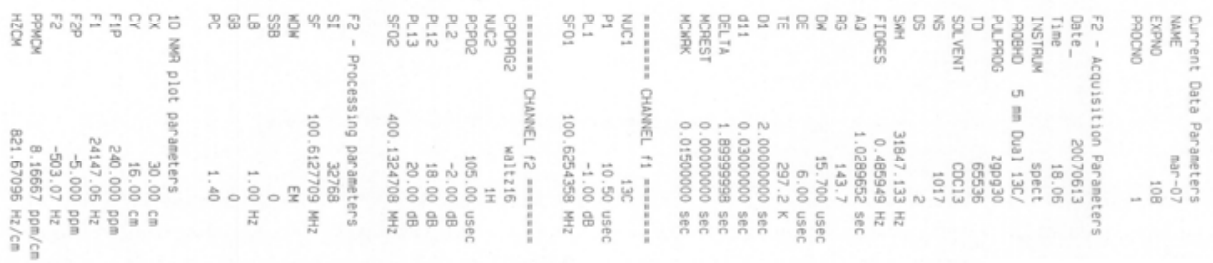




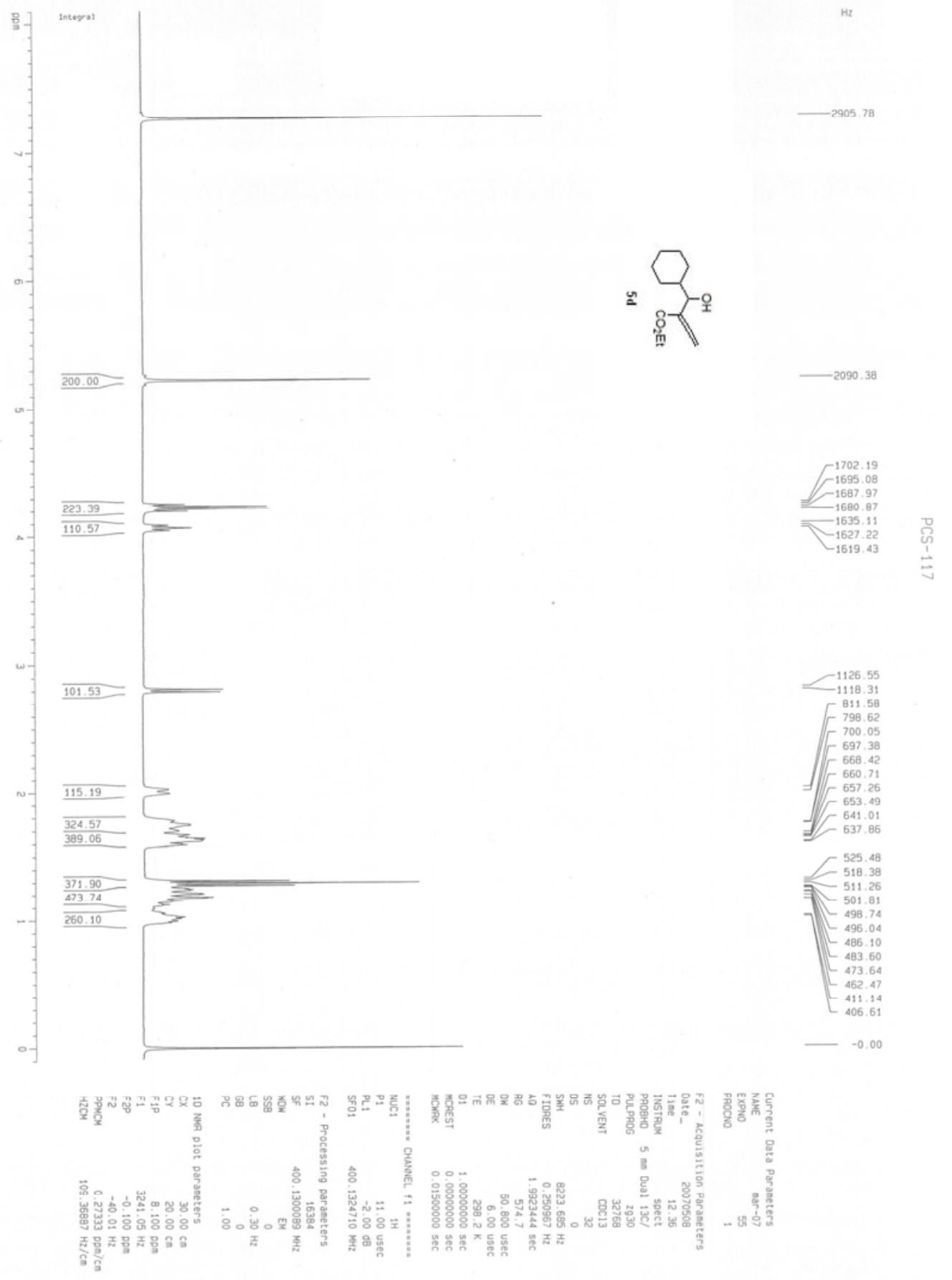



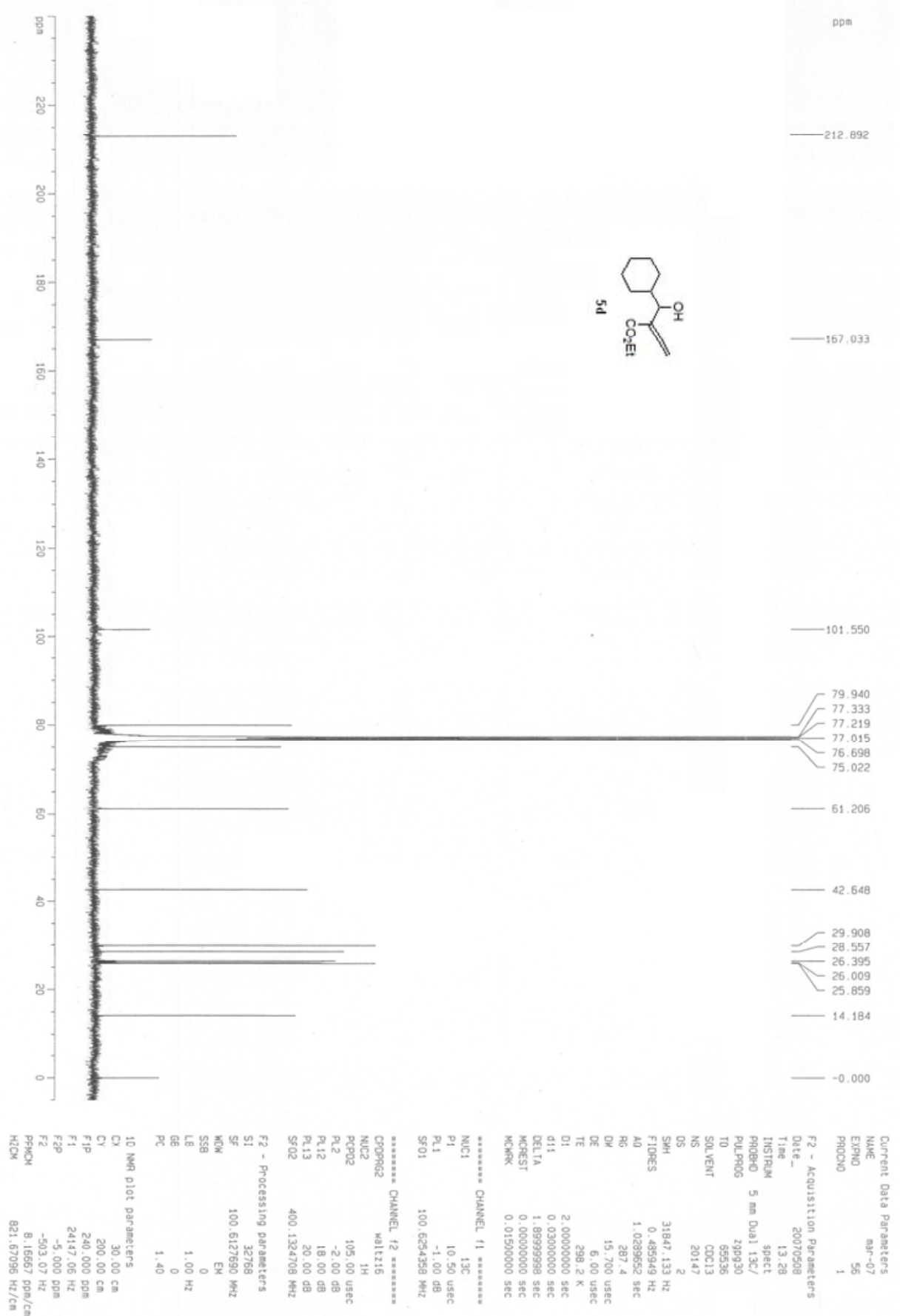


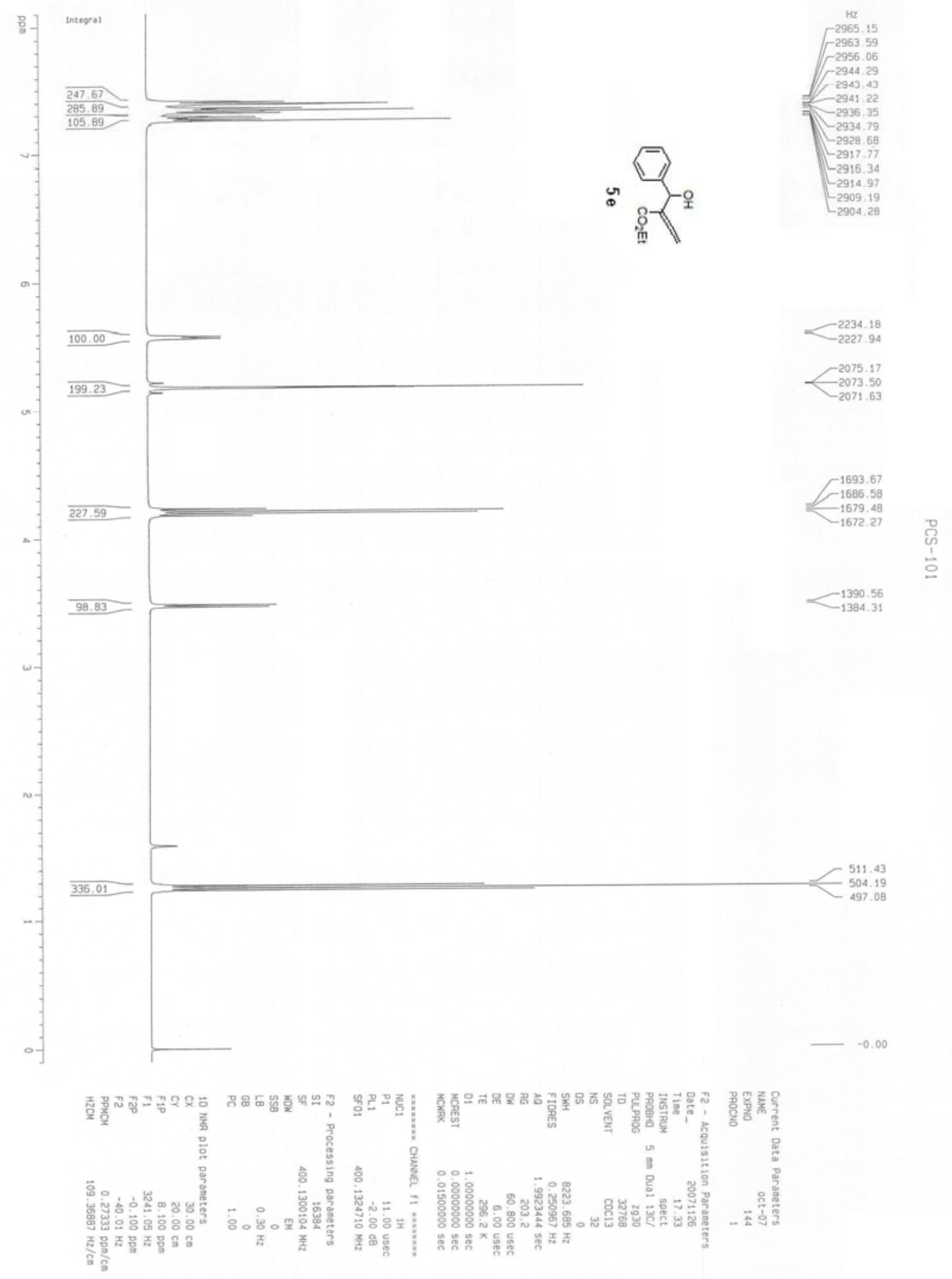



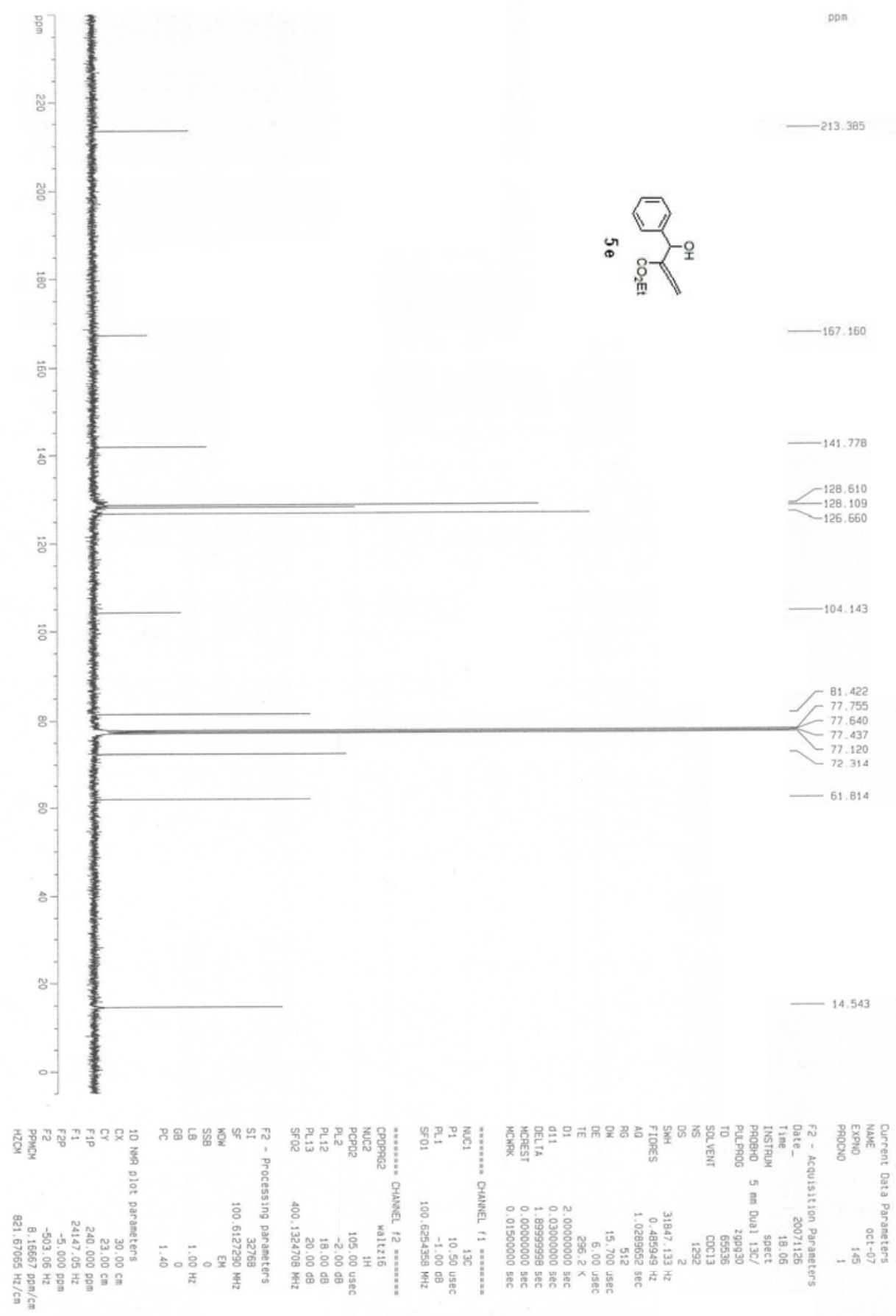


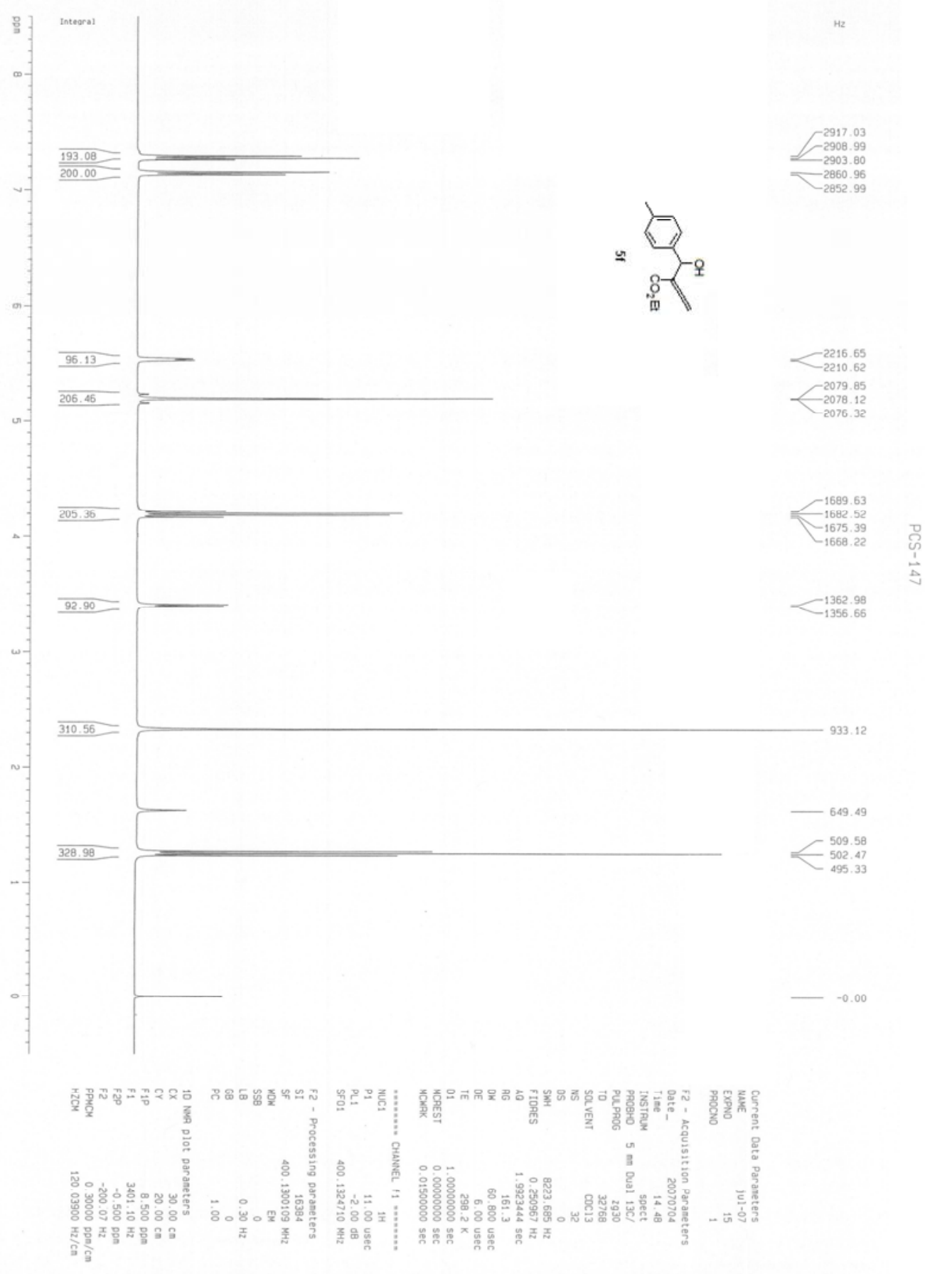




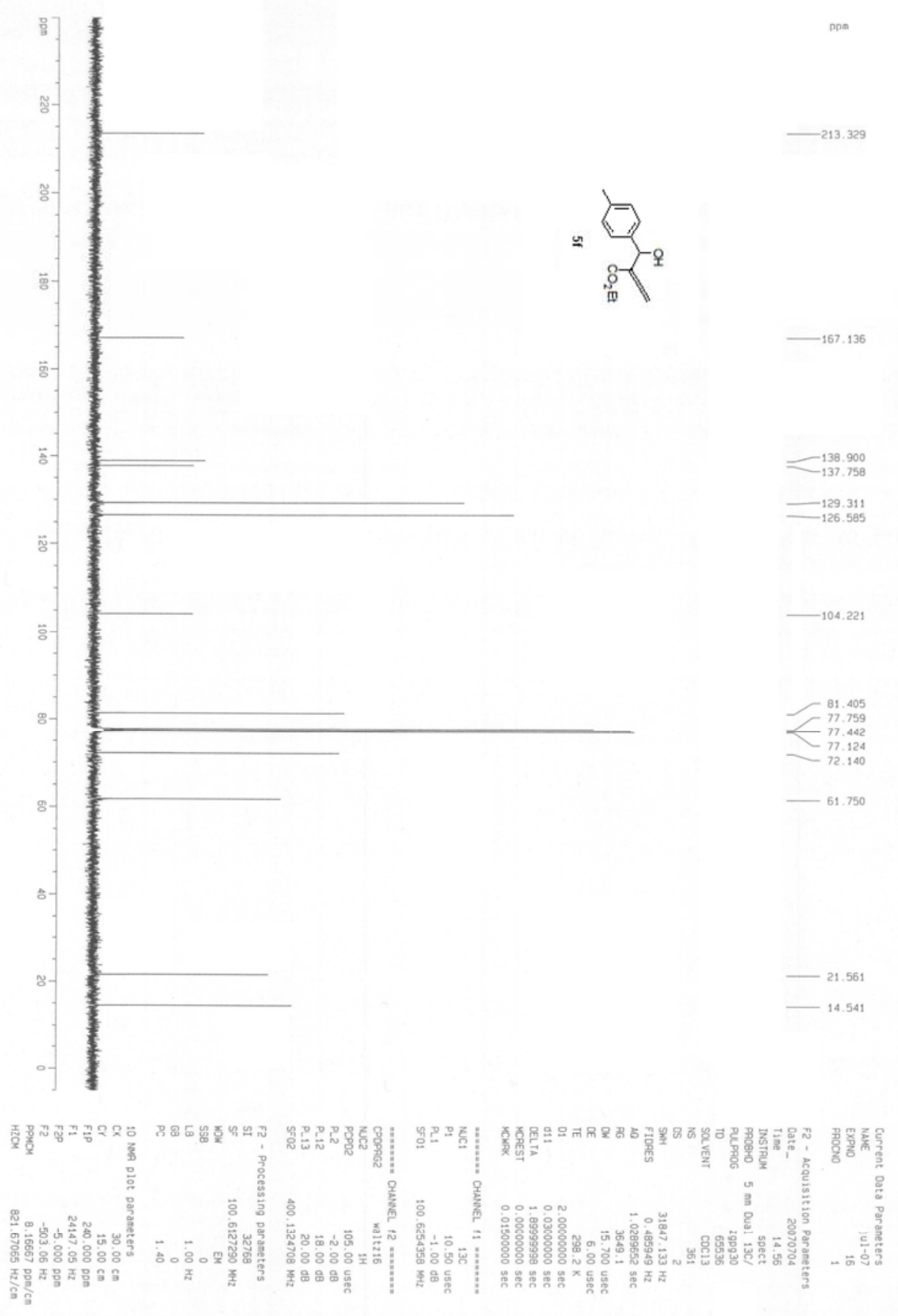




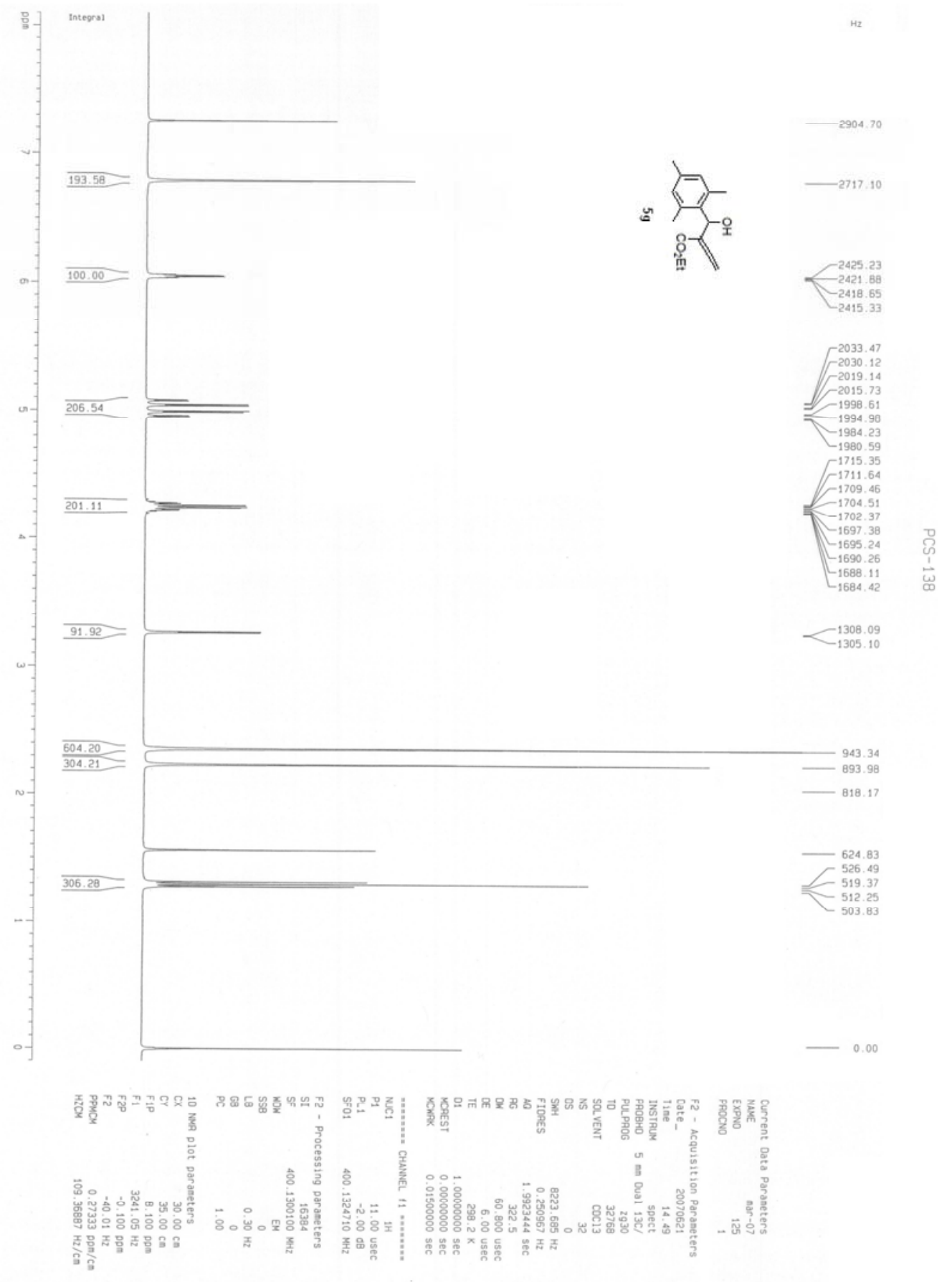



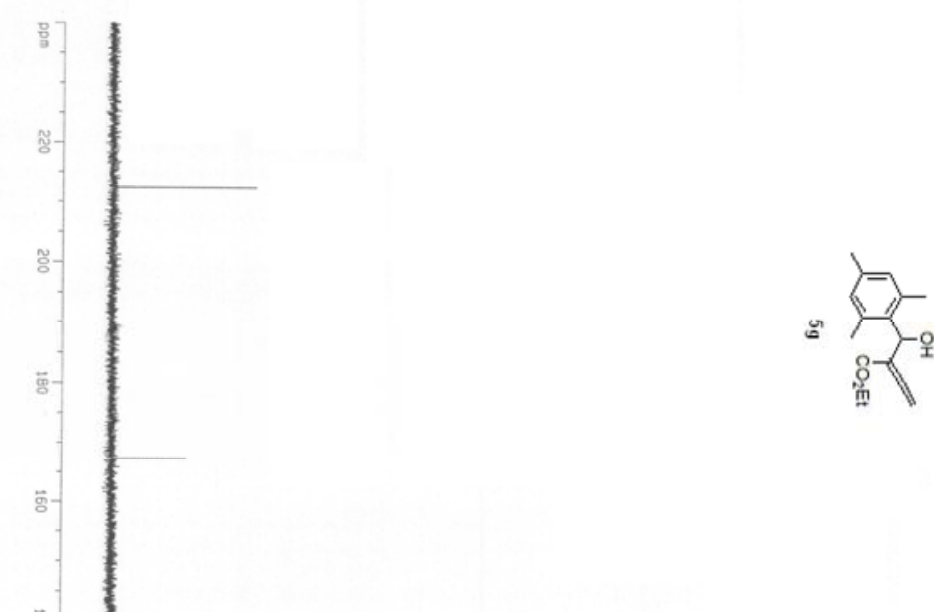

$\tau_{-136.886}^{136.926}$ 132.707
-129.663
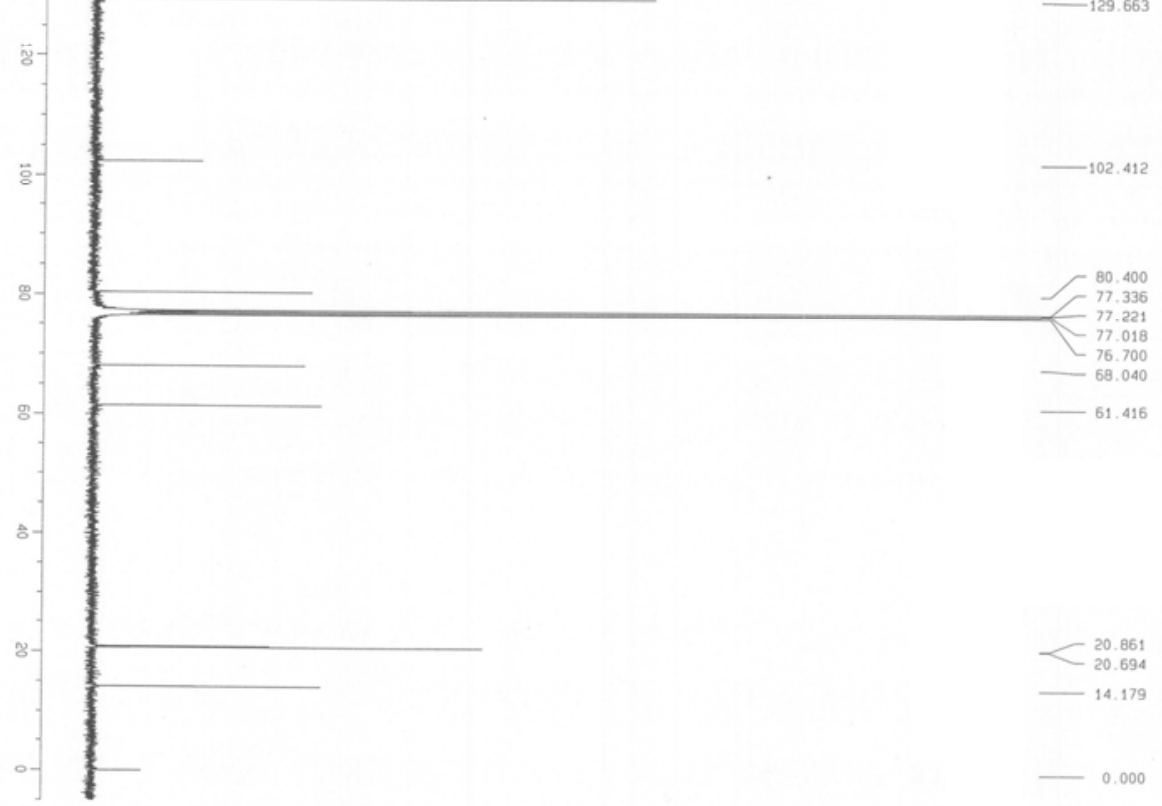

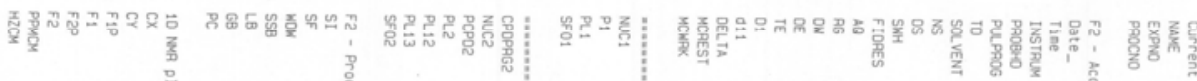

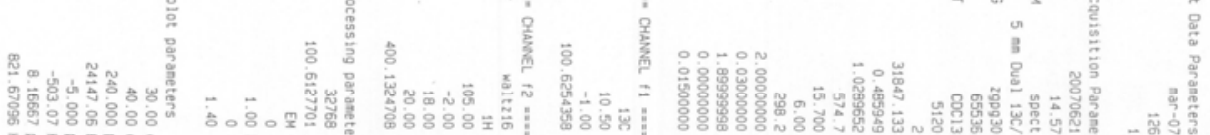

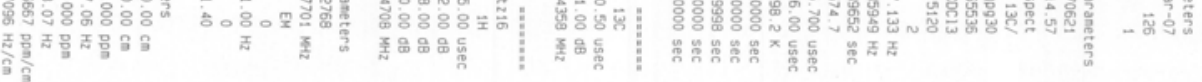




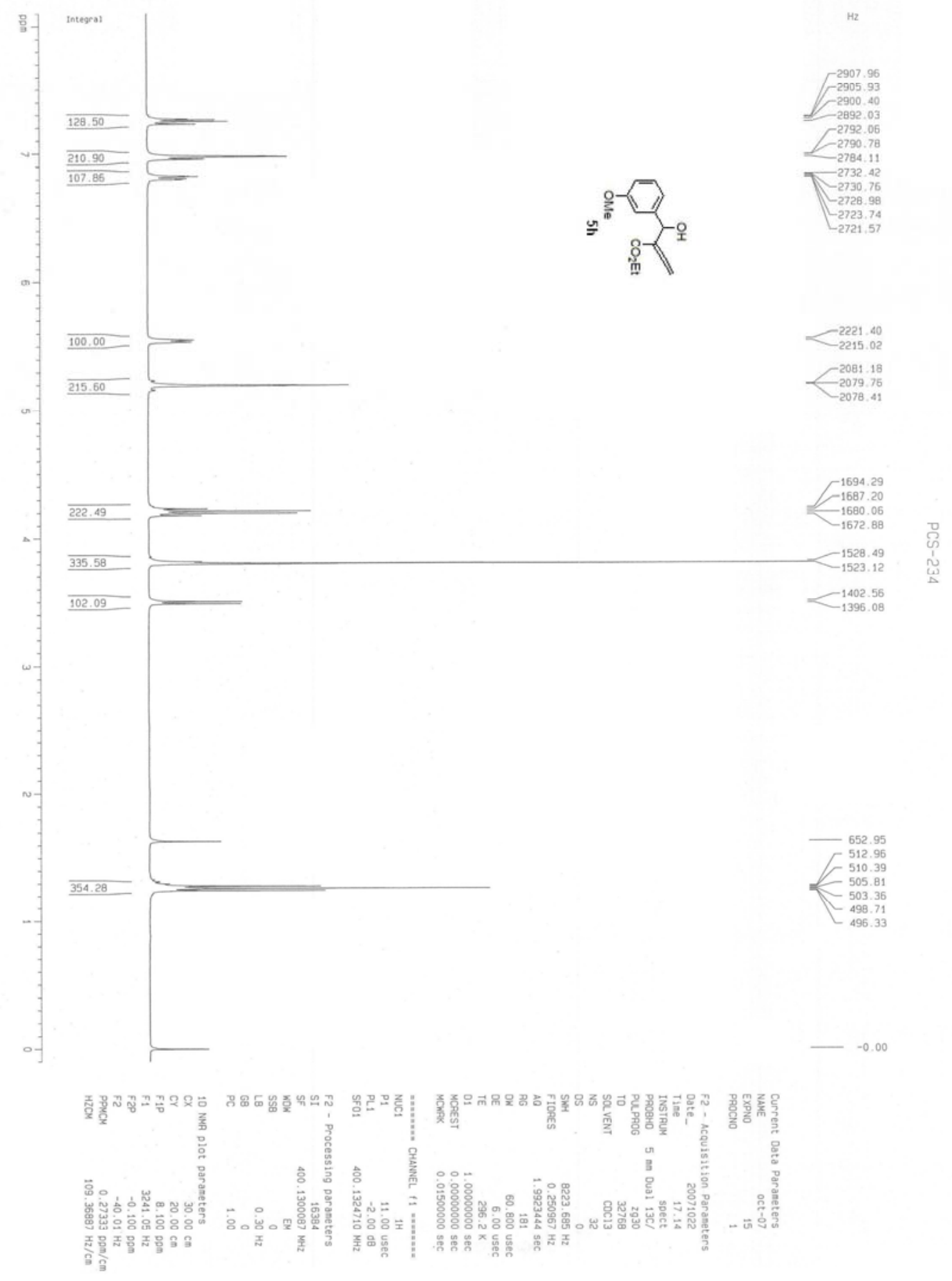

- S30 - 


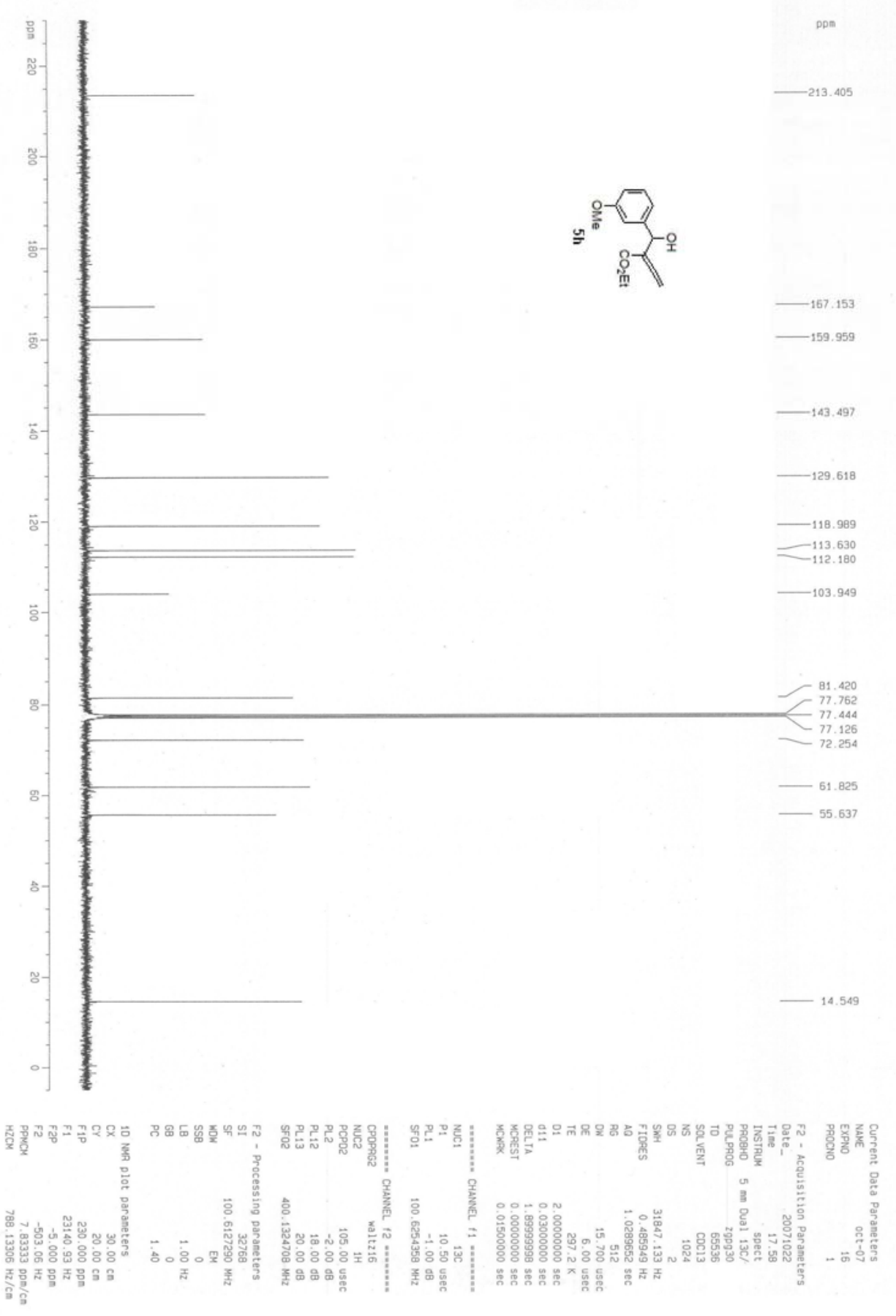




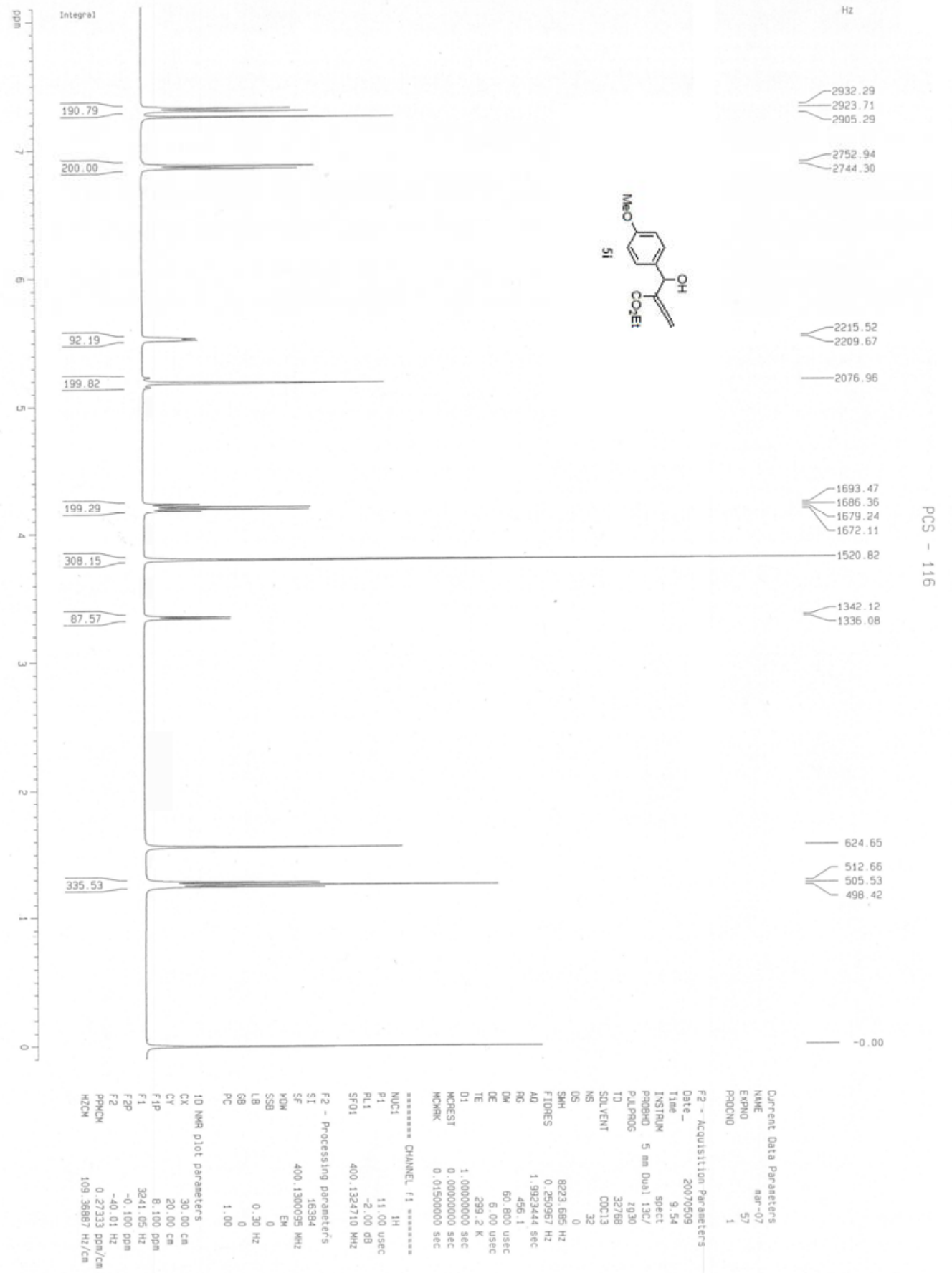




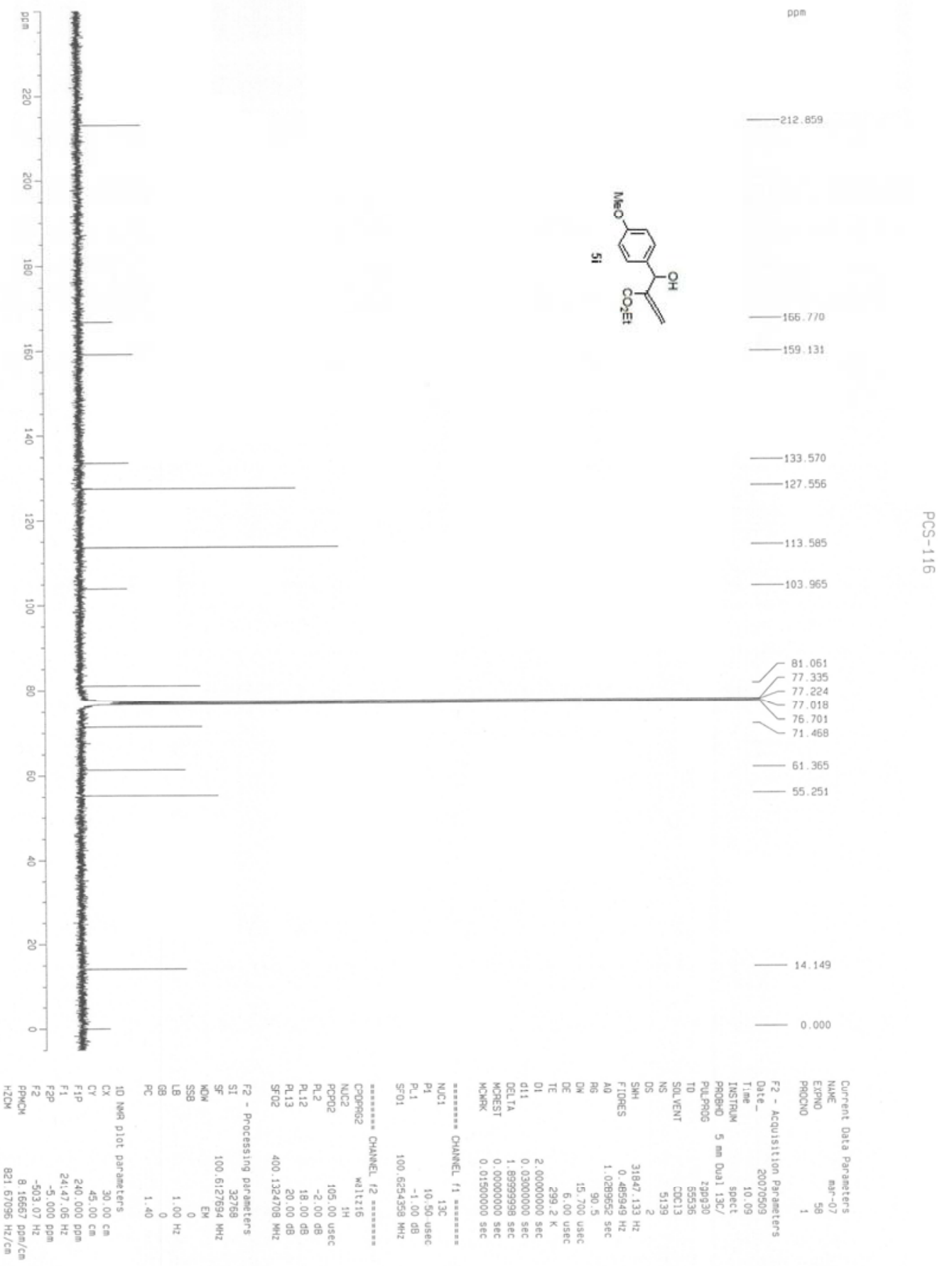




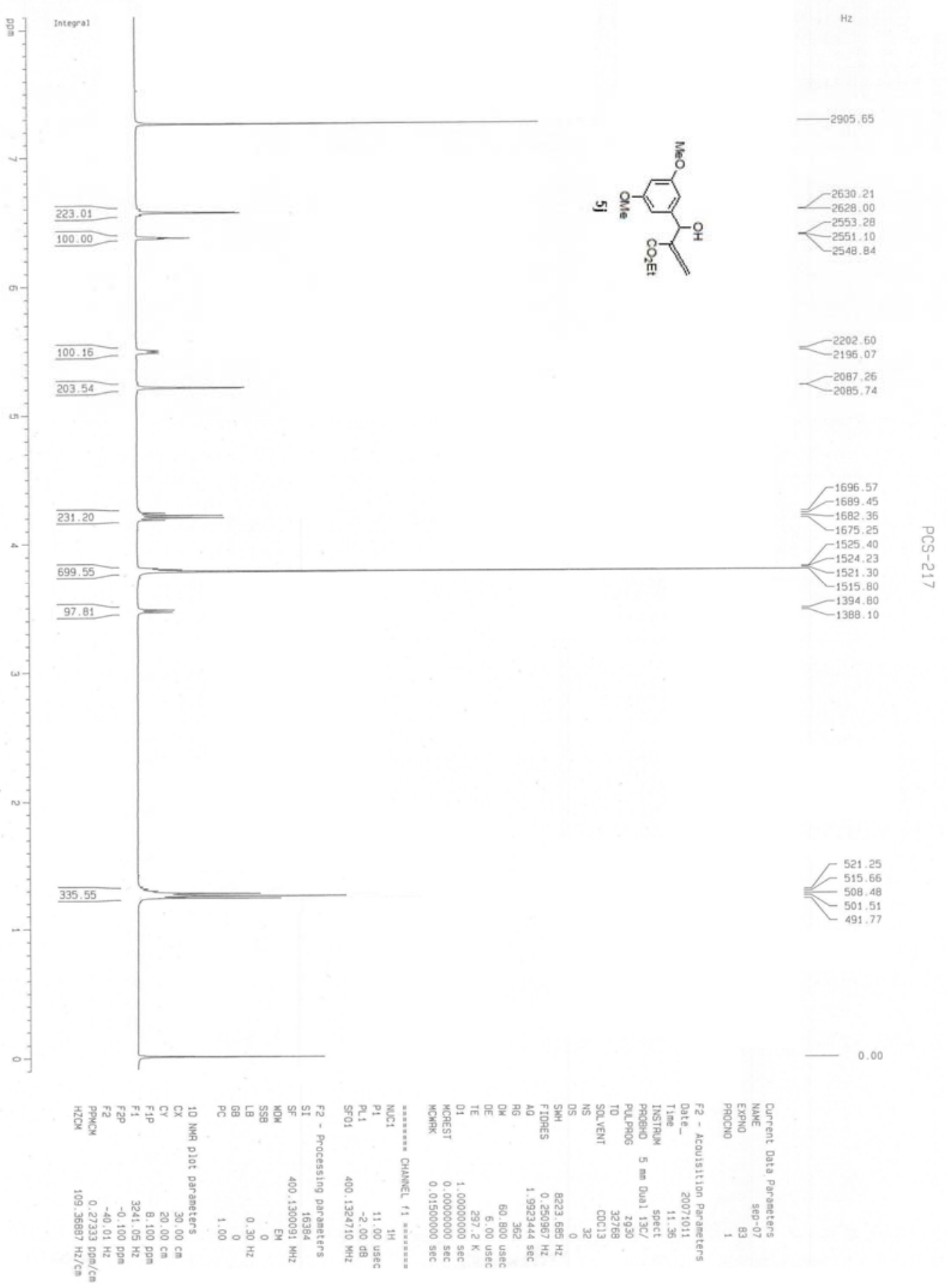




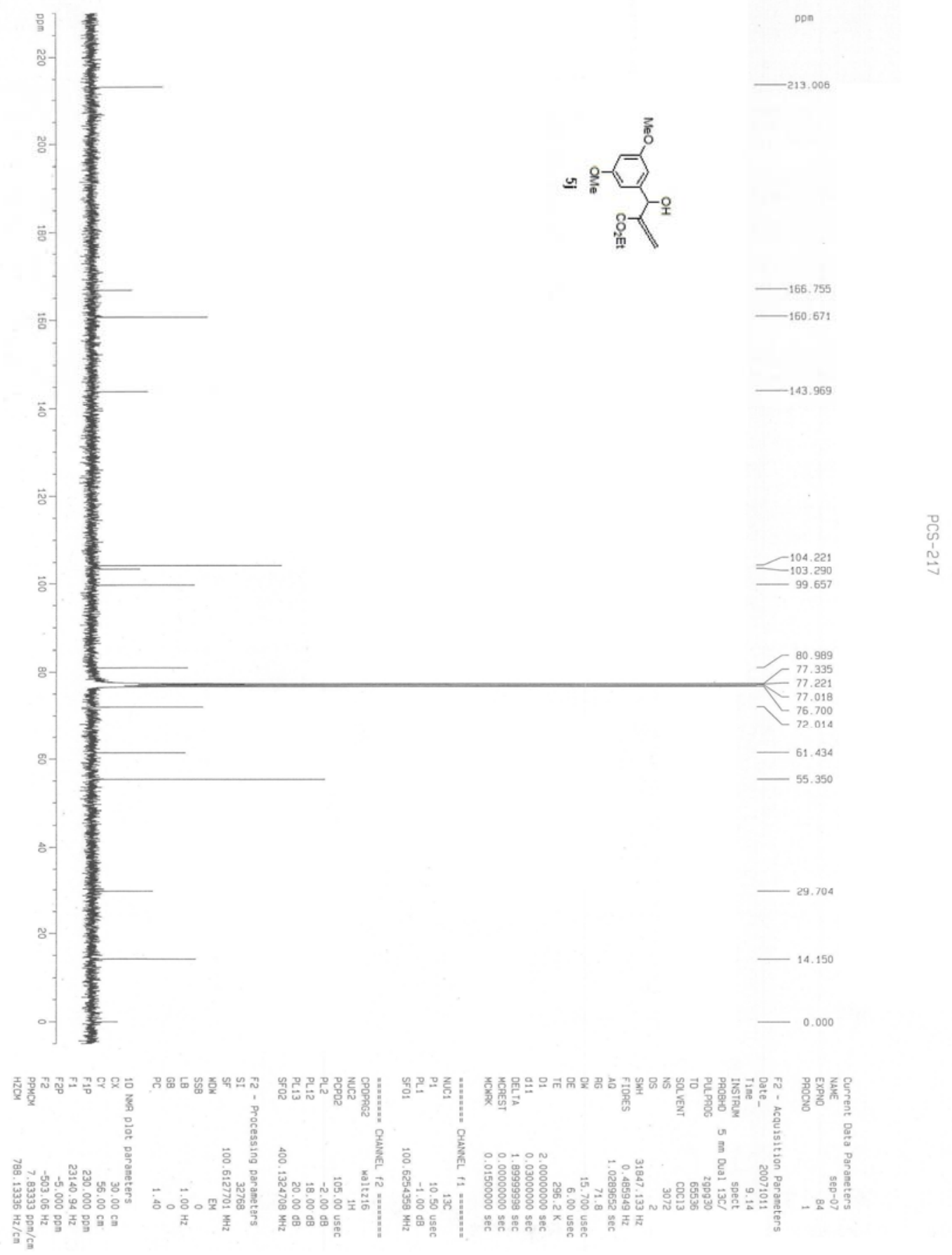




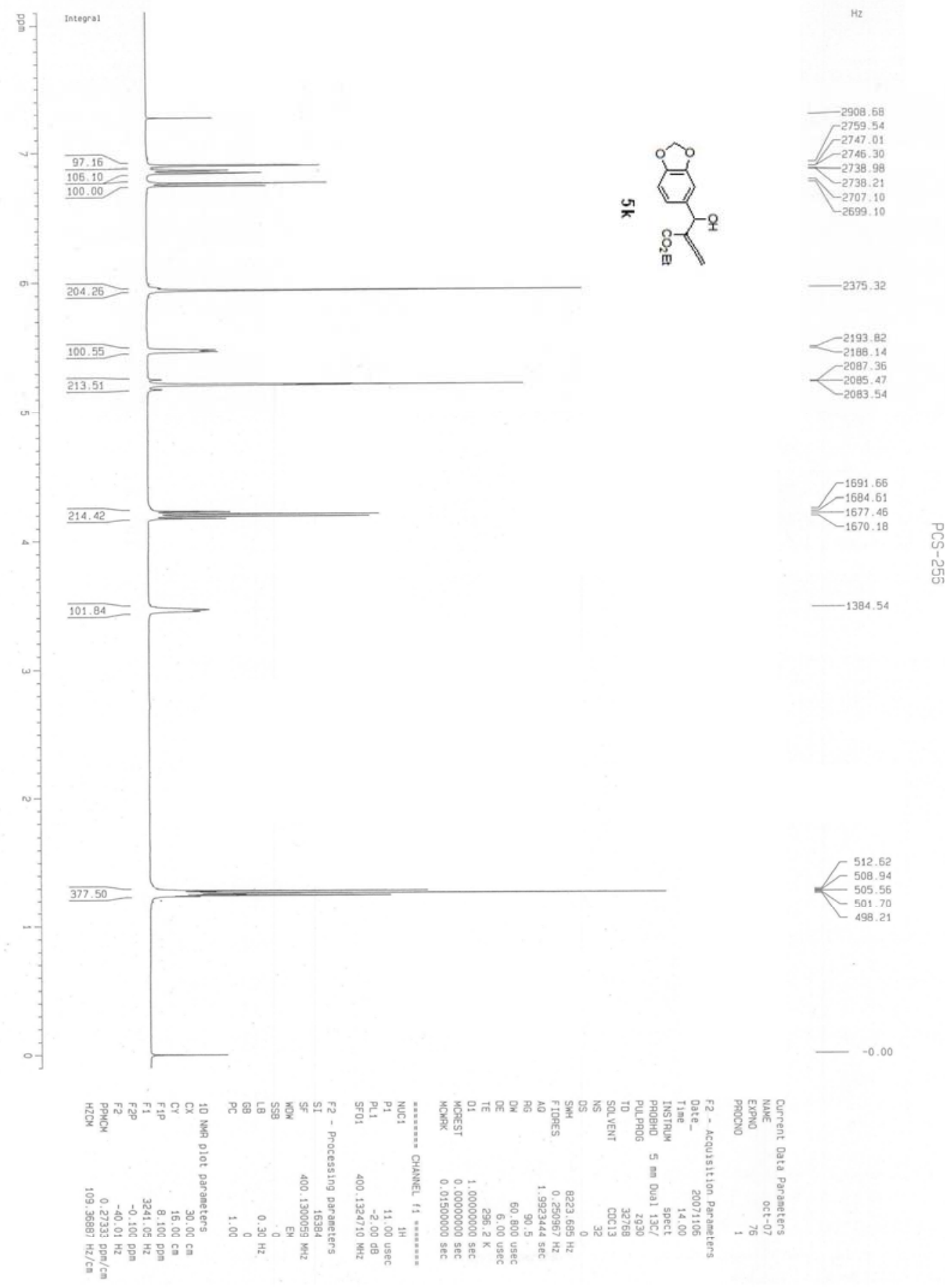



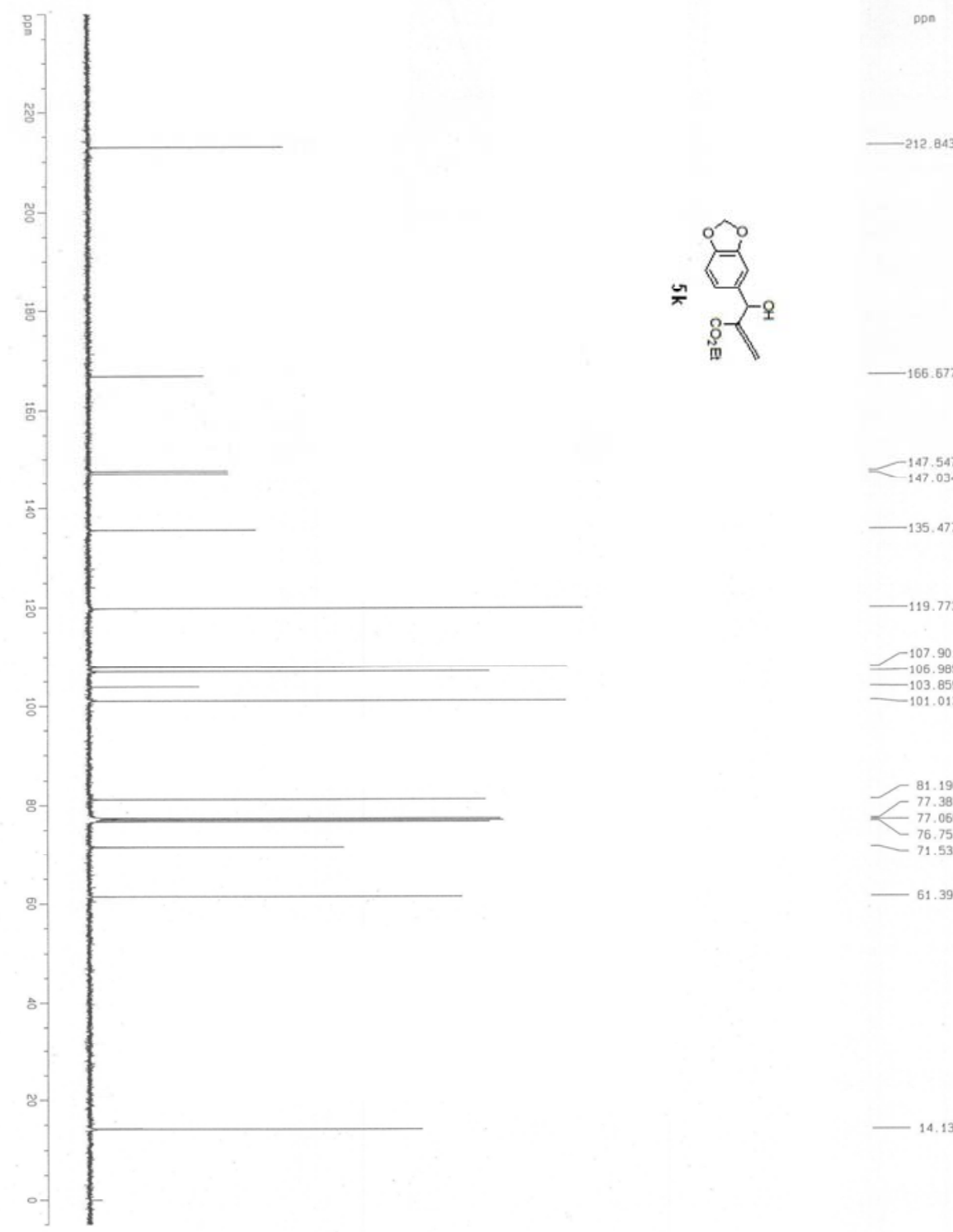

147.547
$-\quad 147.034$

$-135.477$

$-119.773$

$\begin{array}{r}107.901 \\ \mathbf{1}_{106.965} \\ \hline\end{array}$

103.859
-101.013

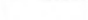

$\begin{array}{r}81.190 \\ <\begin{array}{r}77.387 \\ \quad 77.069 \\ 76.751 \\ \hline\end{array} 71.532 \\ \hline\end{array}$

- 61.390

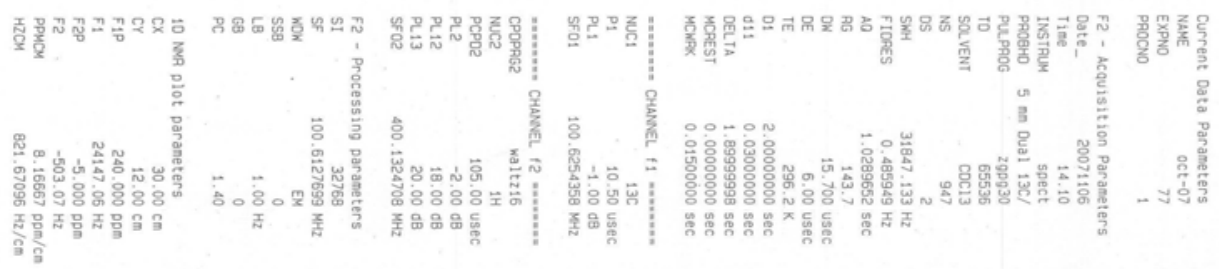




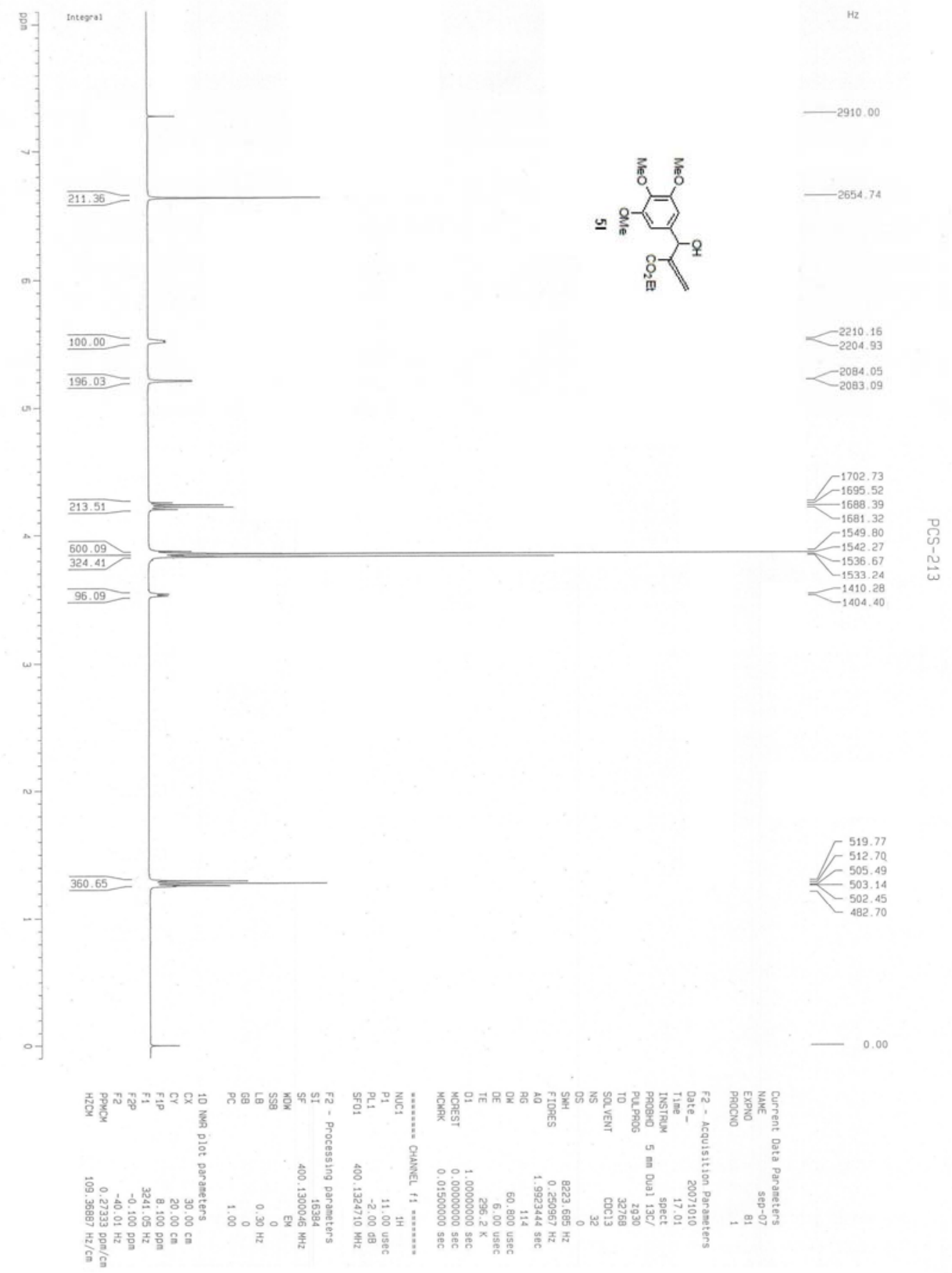




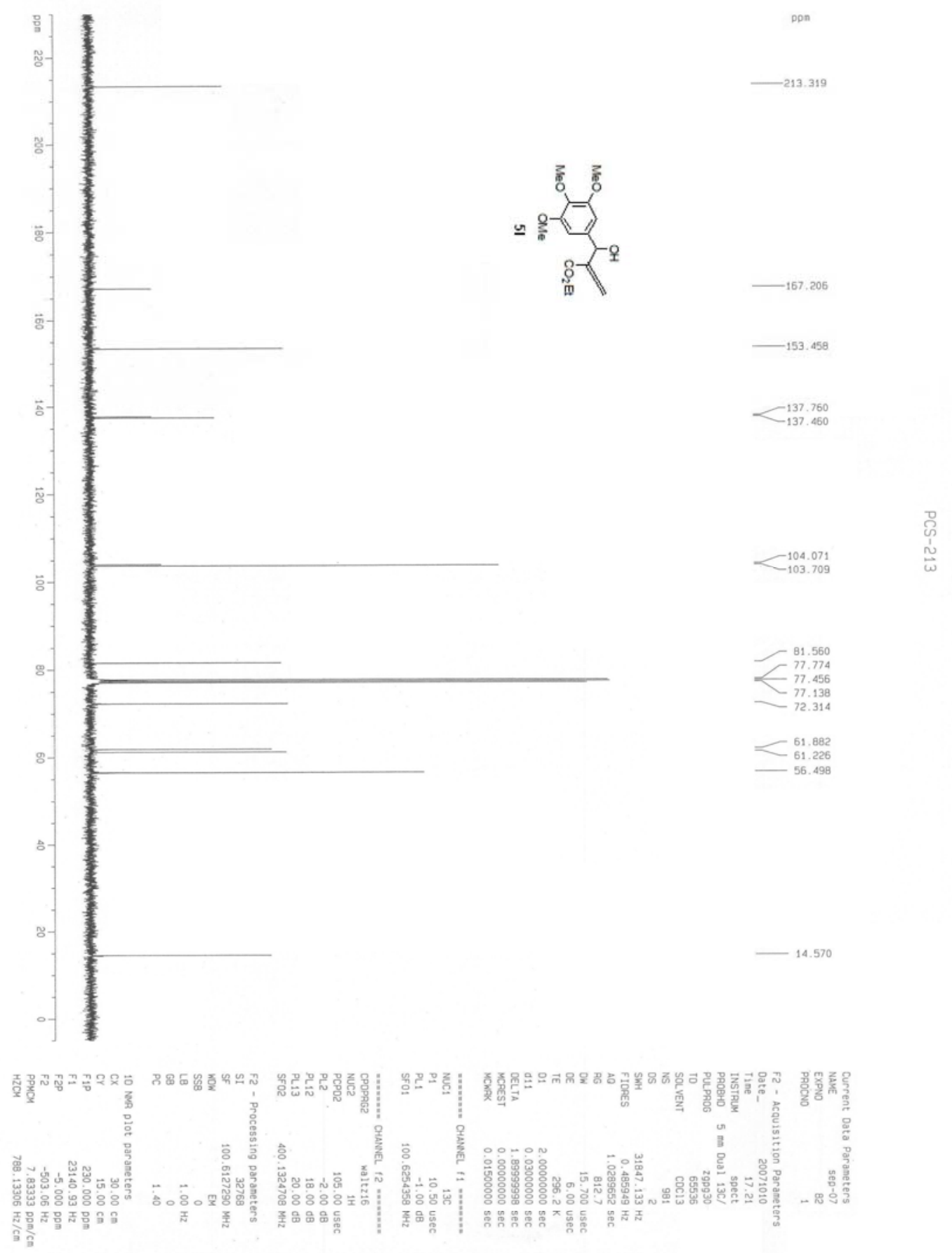




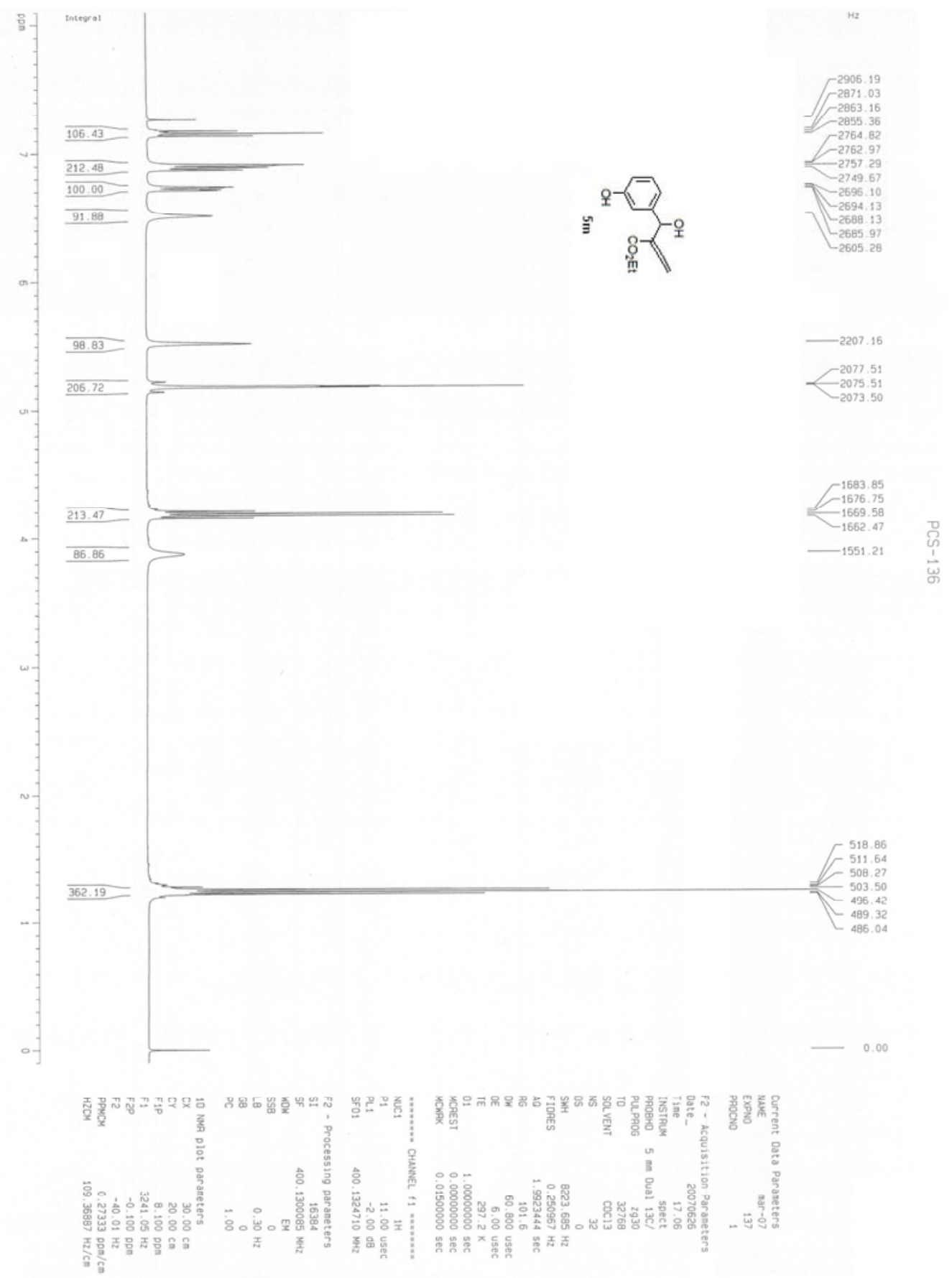



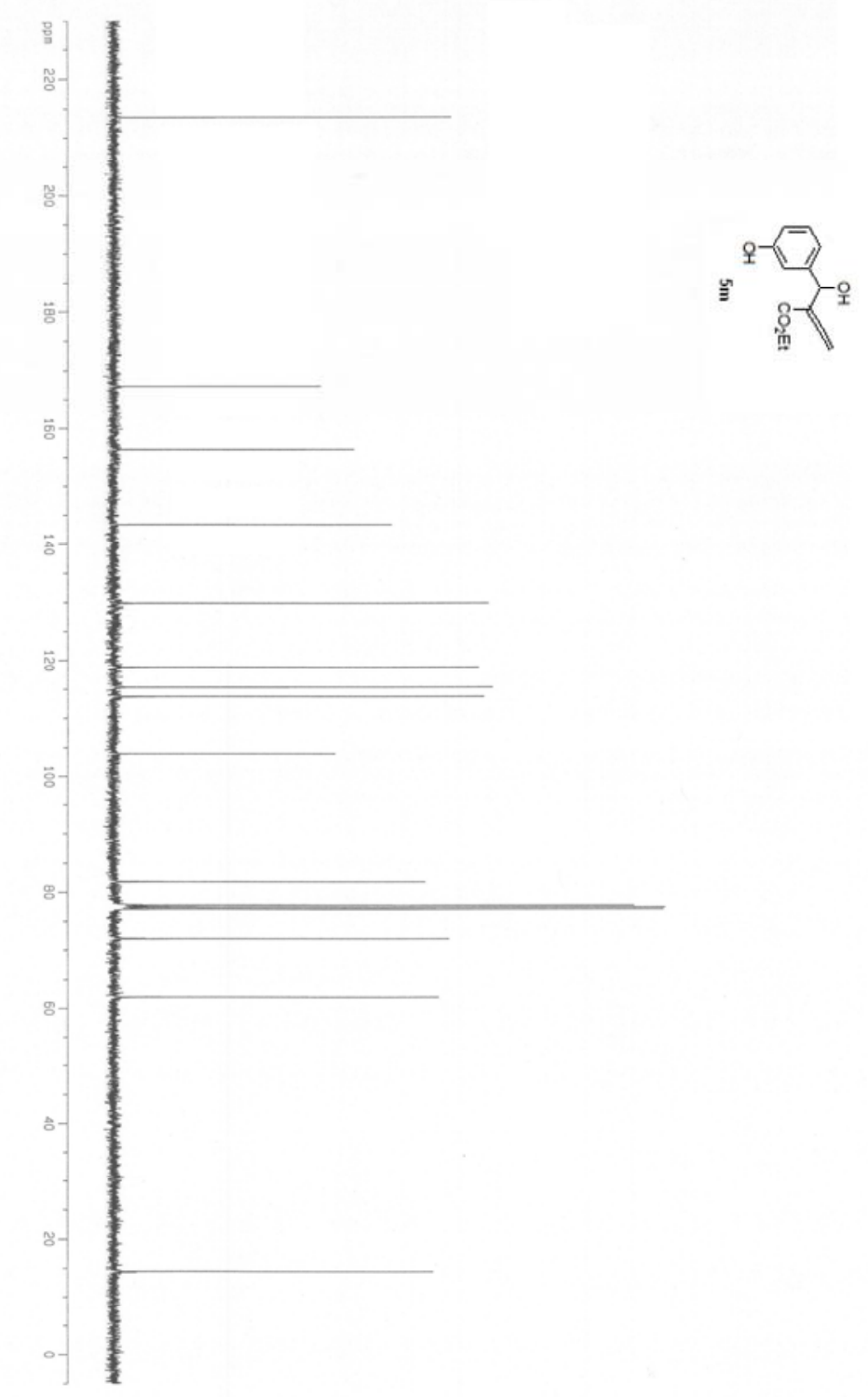

$-213.531$

$-167.300$

$-156.323$

$-143.392$

$-129.878$

$\begin{array}{r}118.797 \\ \square \\ \hline \\ \hline\end{array} 15.349$

$-103.894$

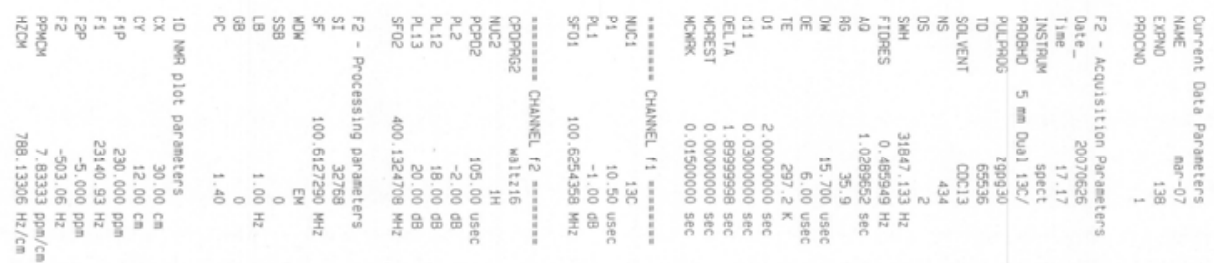




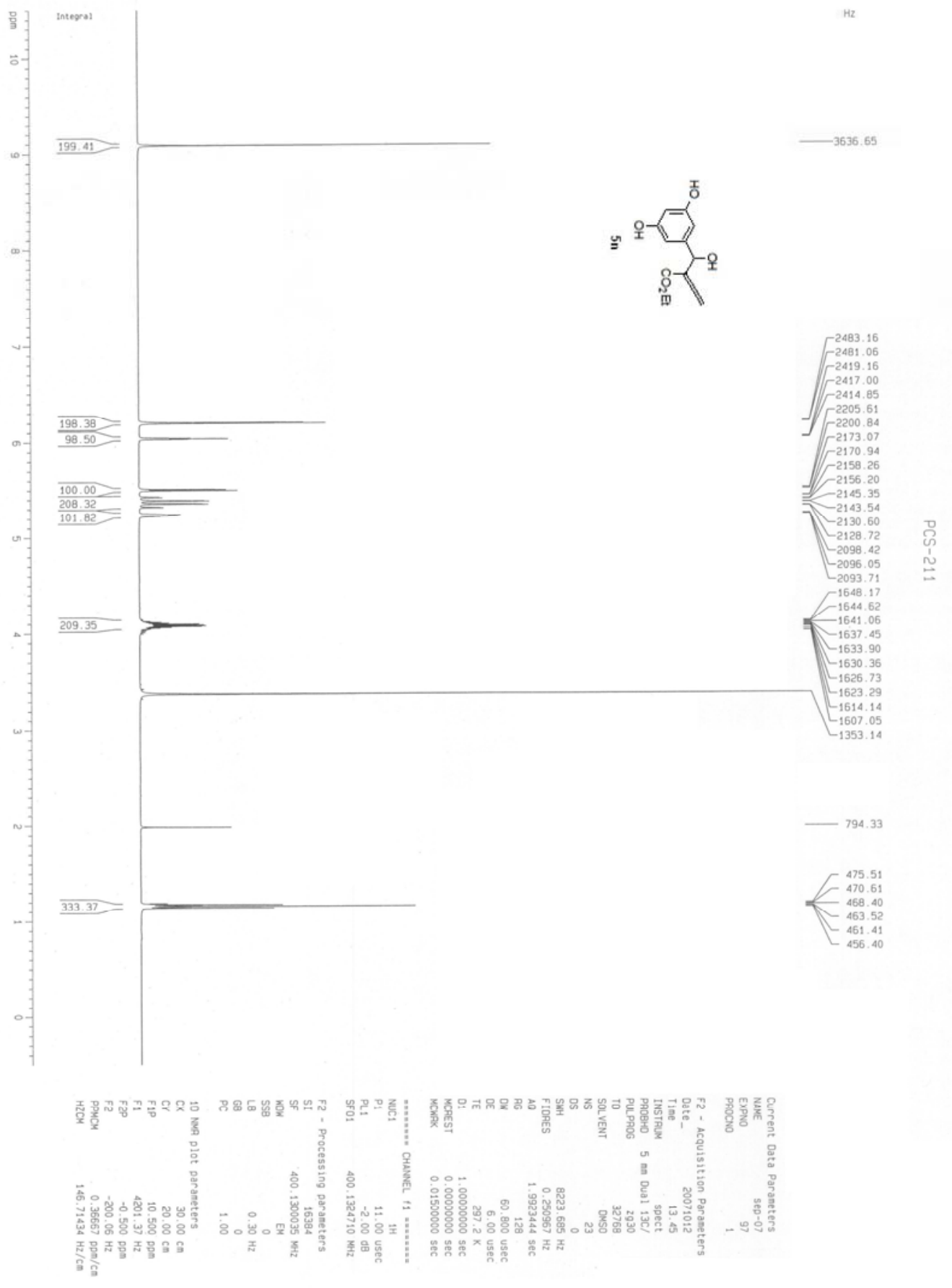



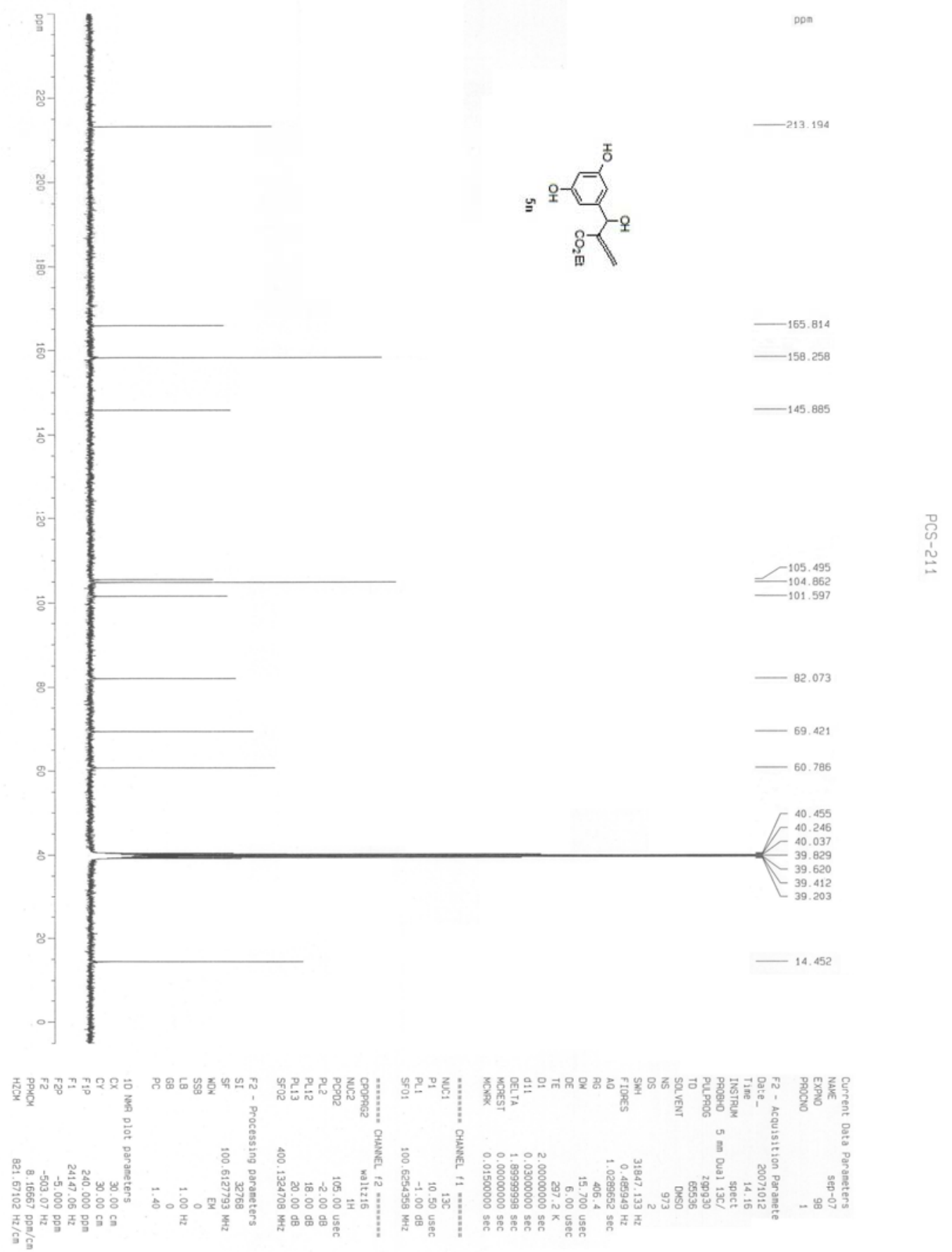


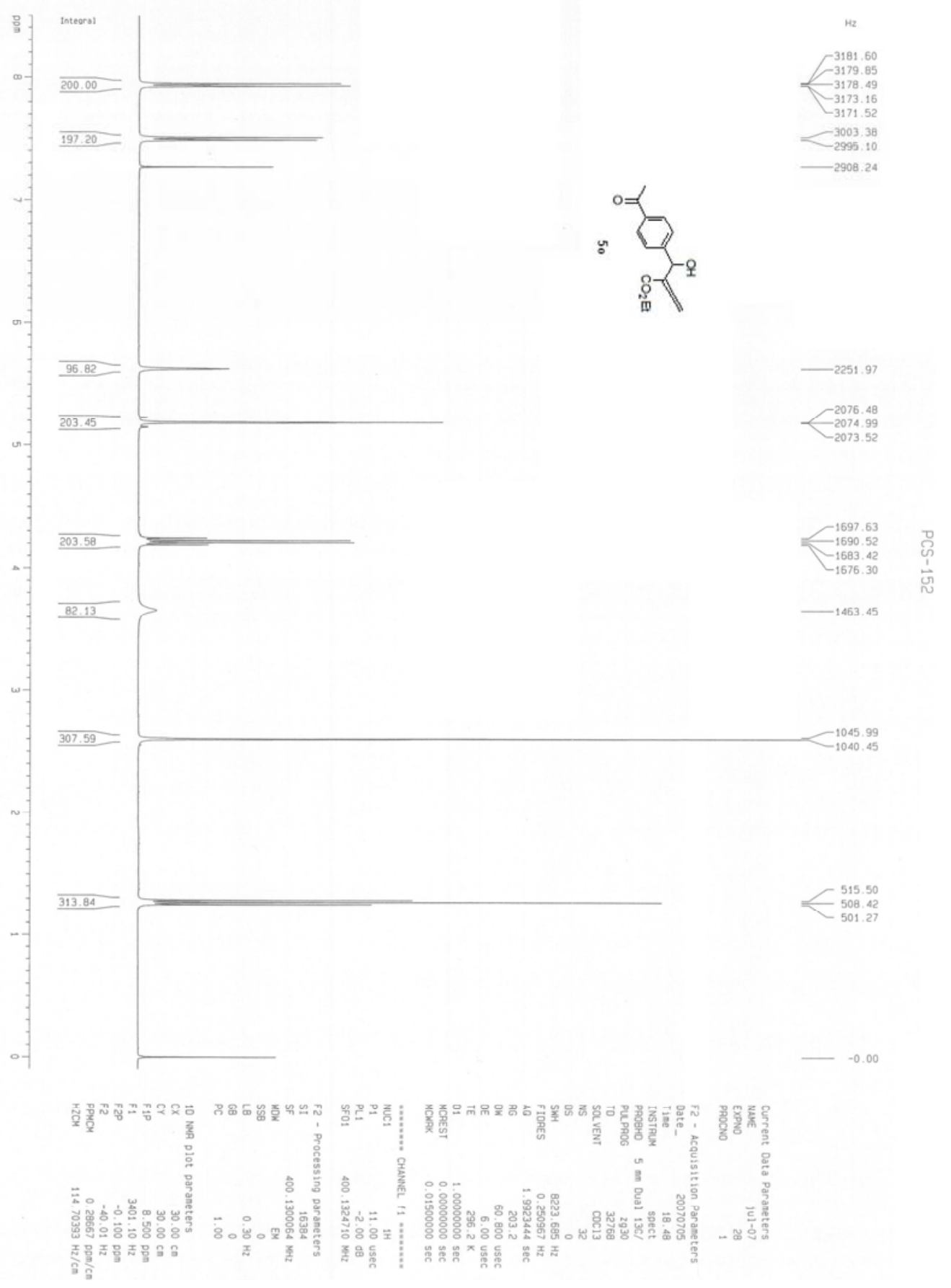




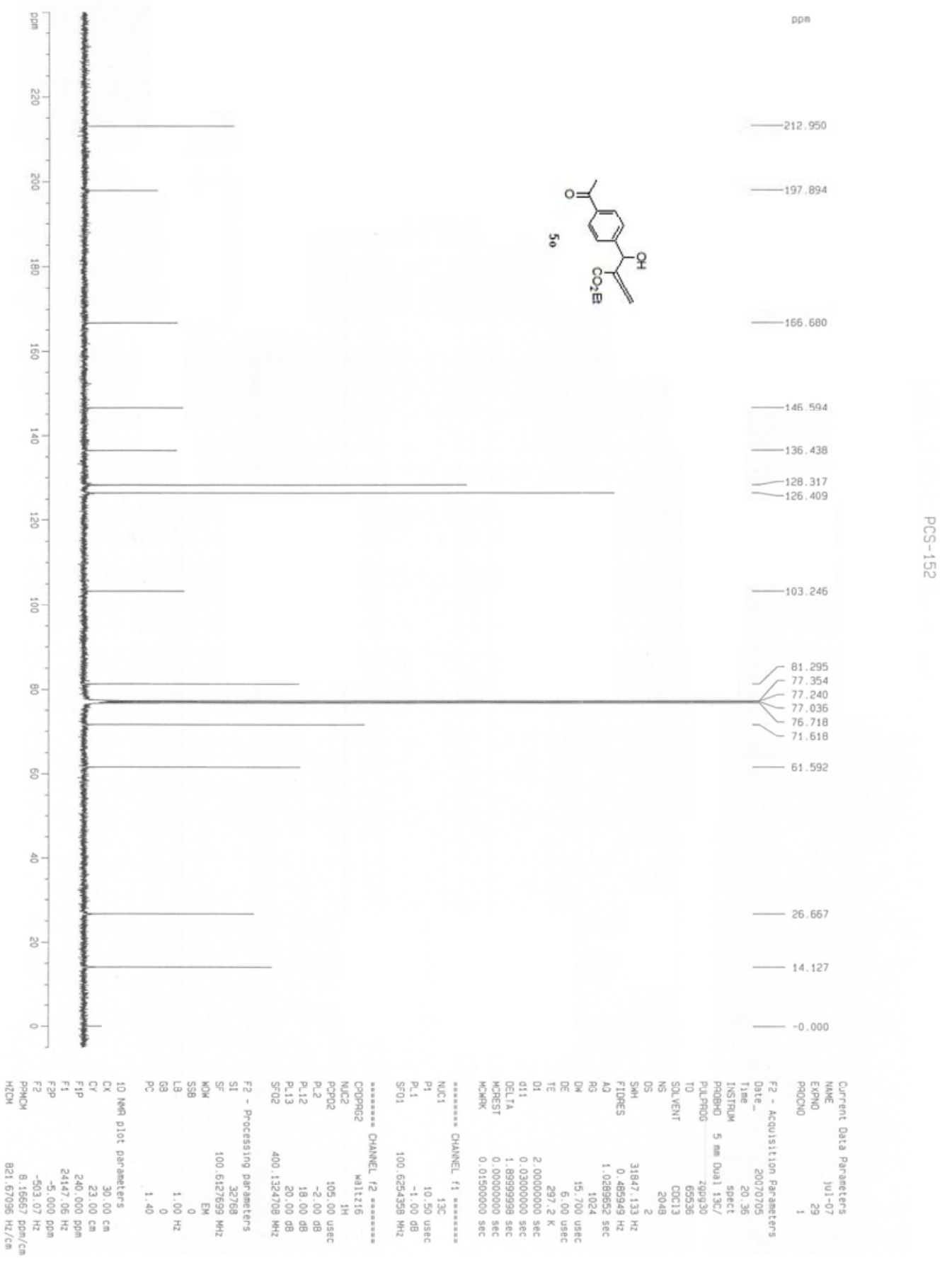




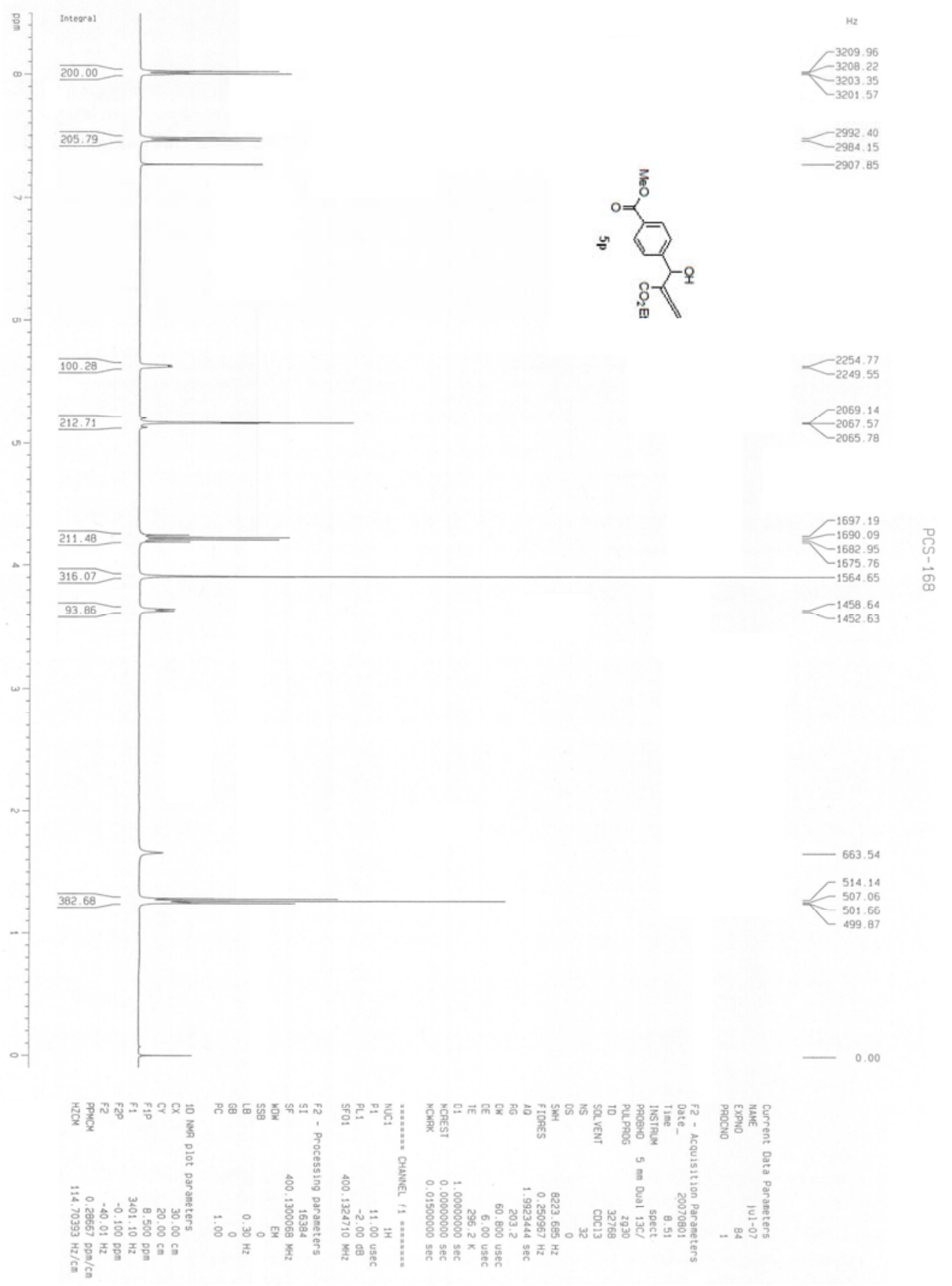



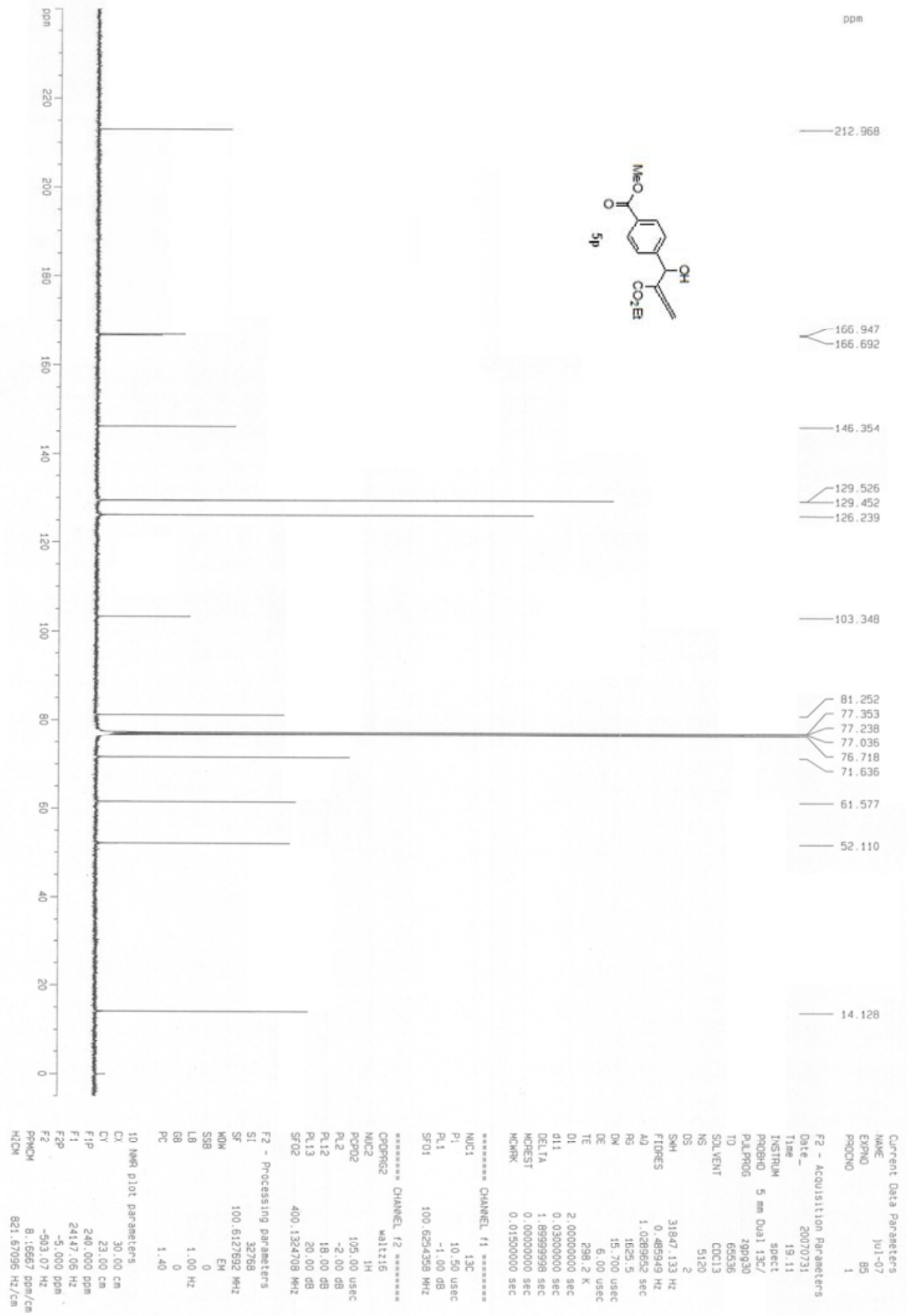


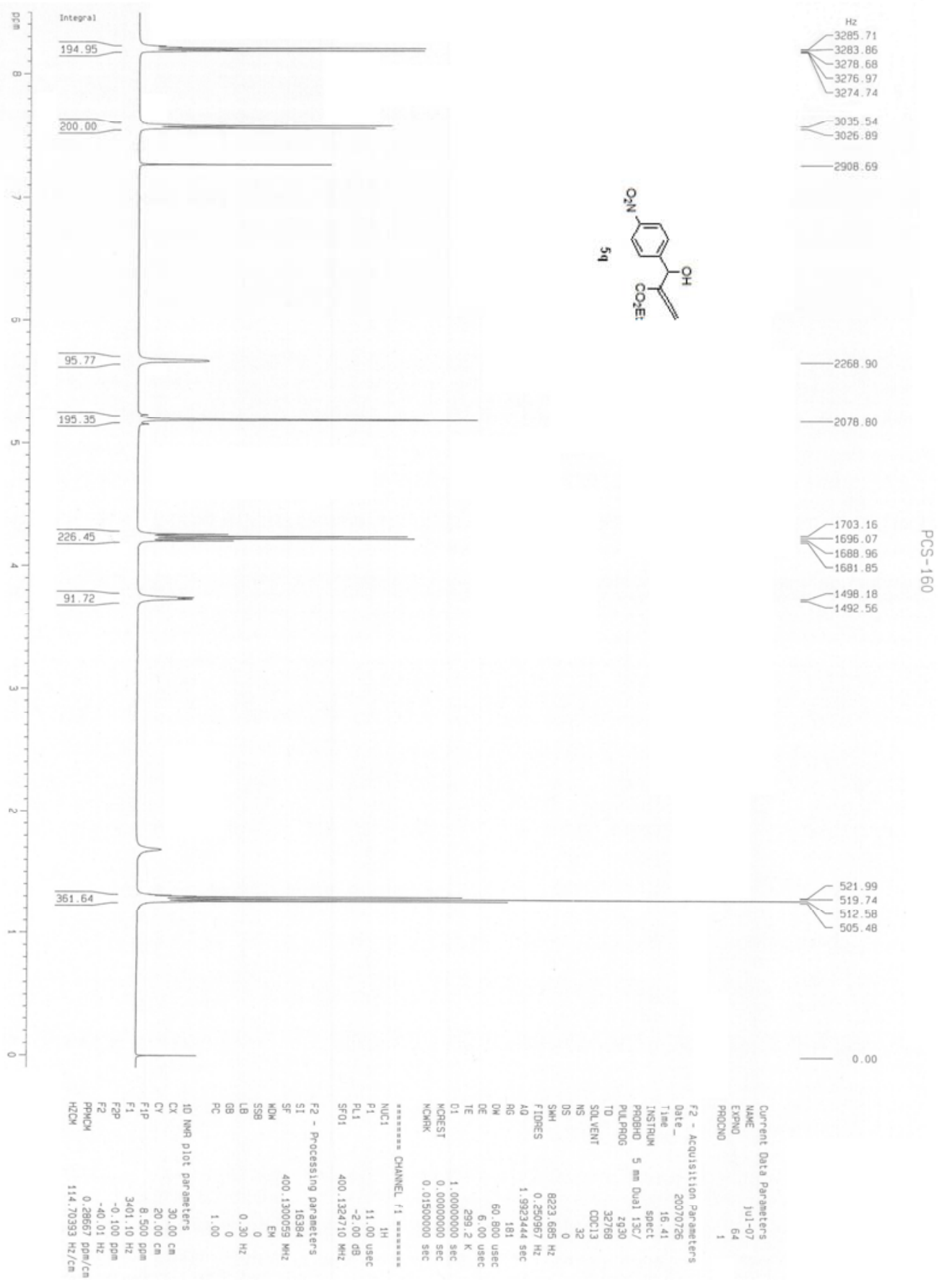



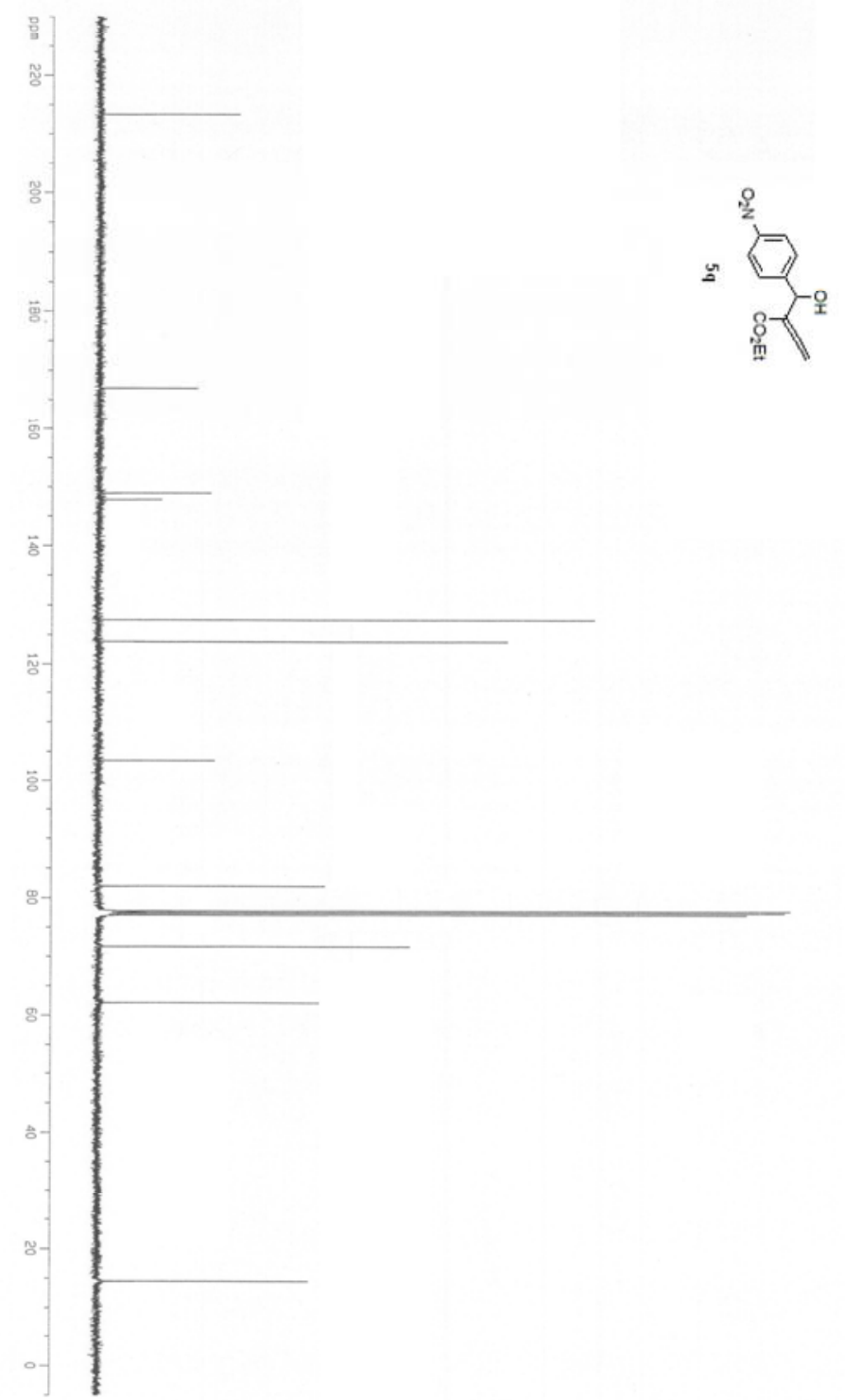

81.978
$\quad 77.755$
-77.640
77.437
77.120
71.648

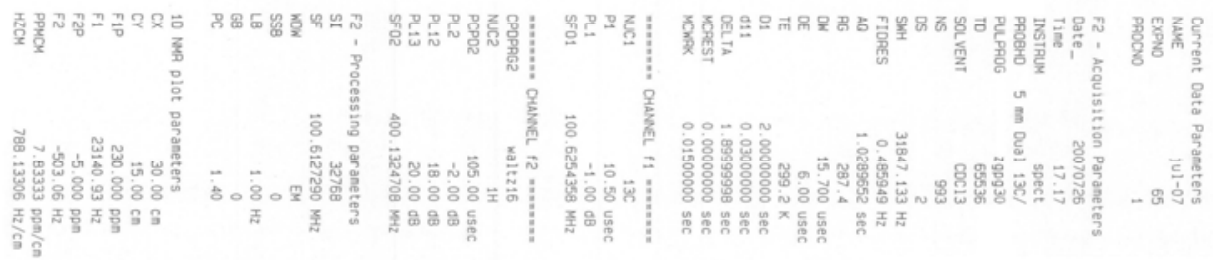




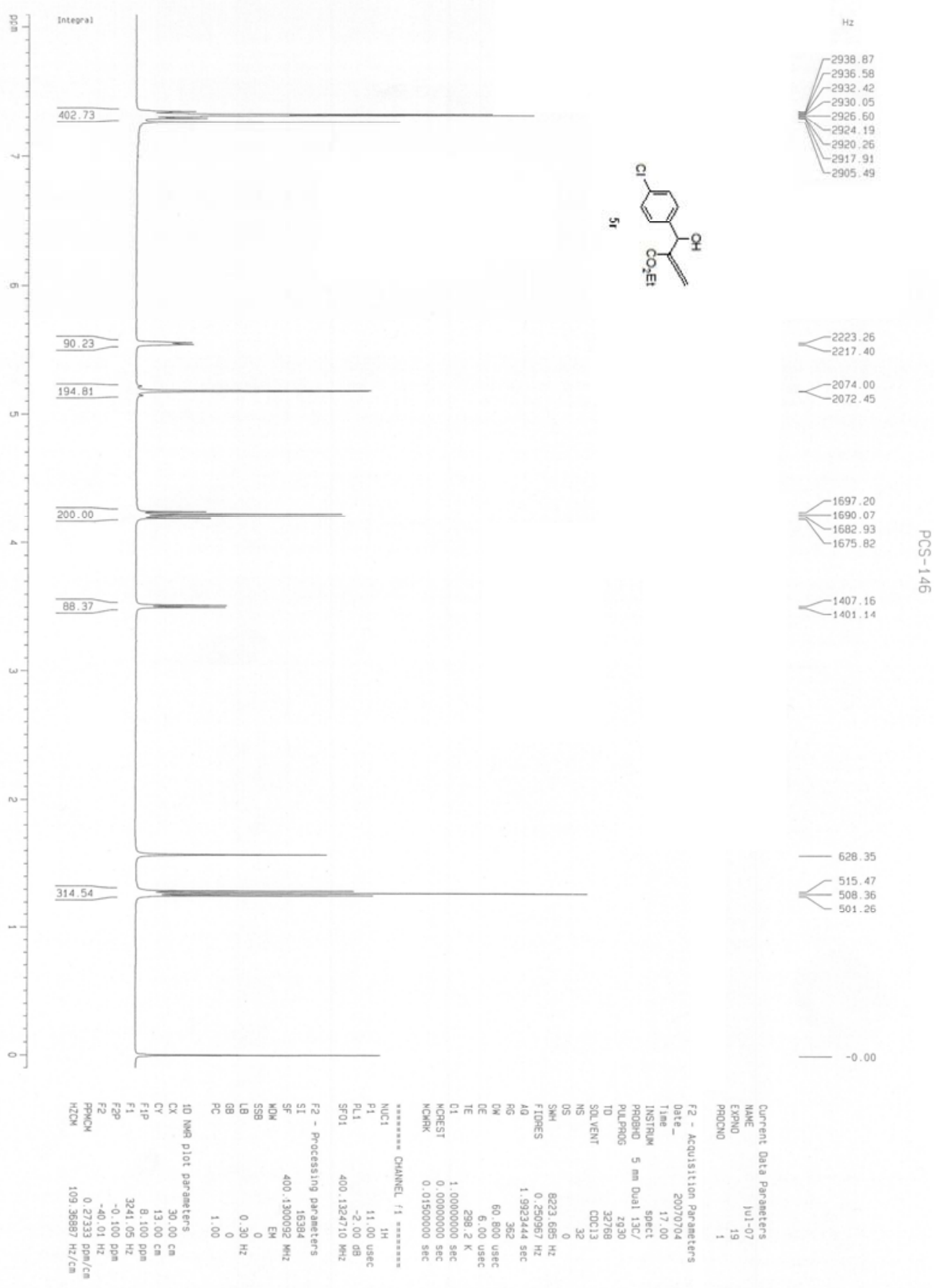

- S50 - 


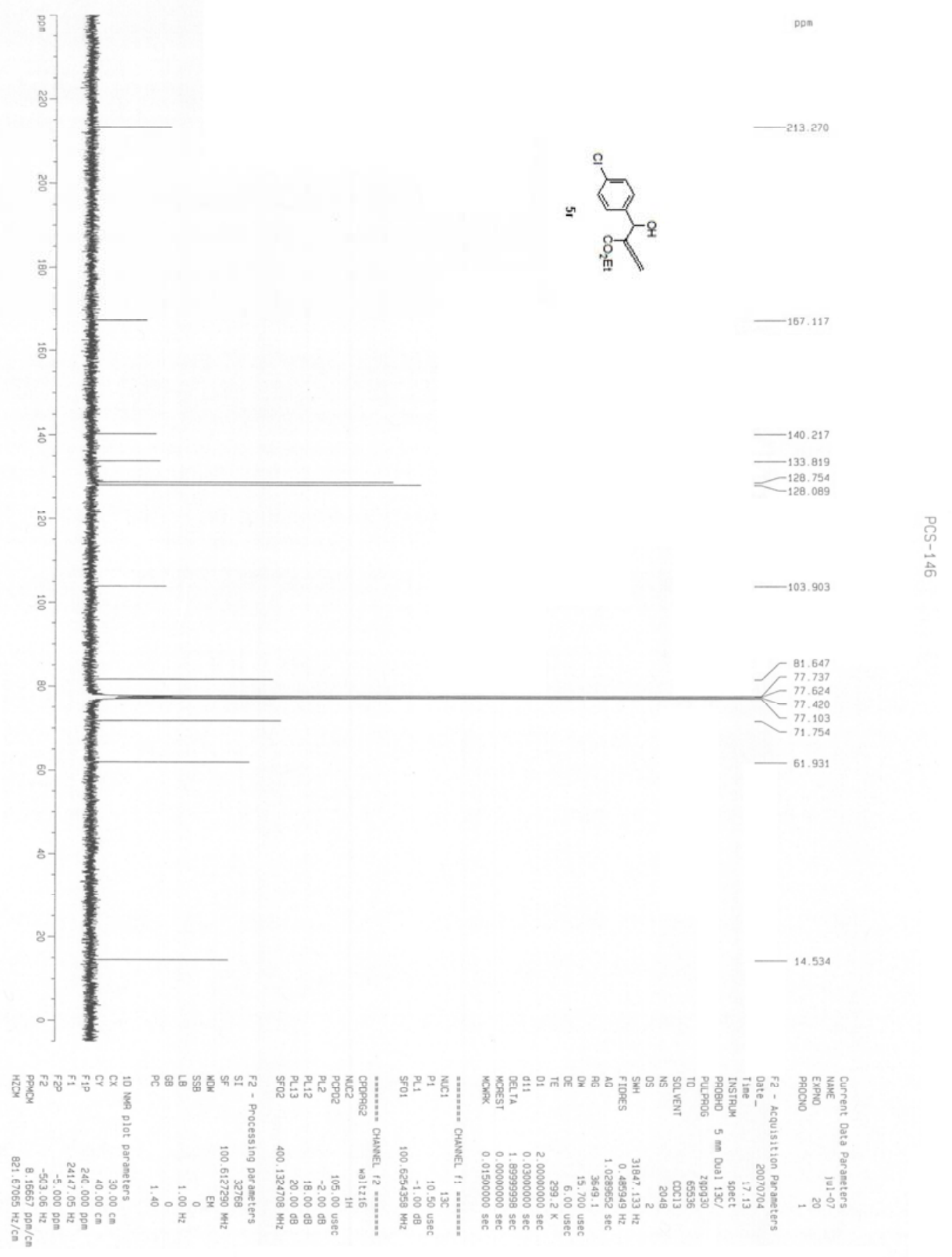




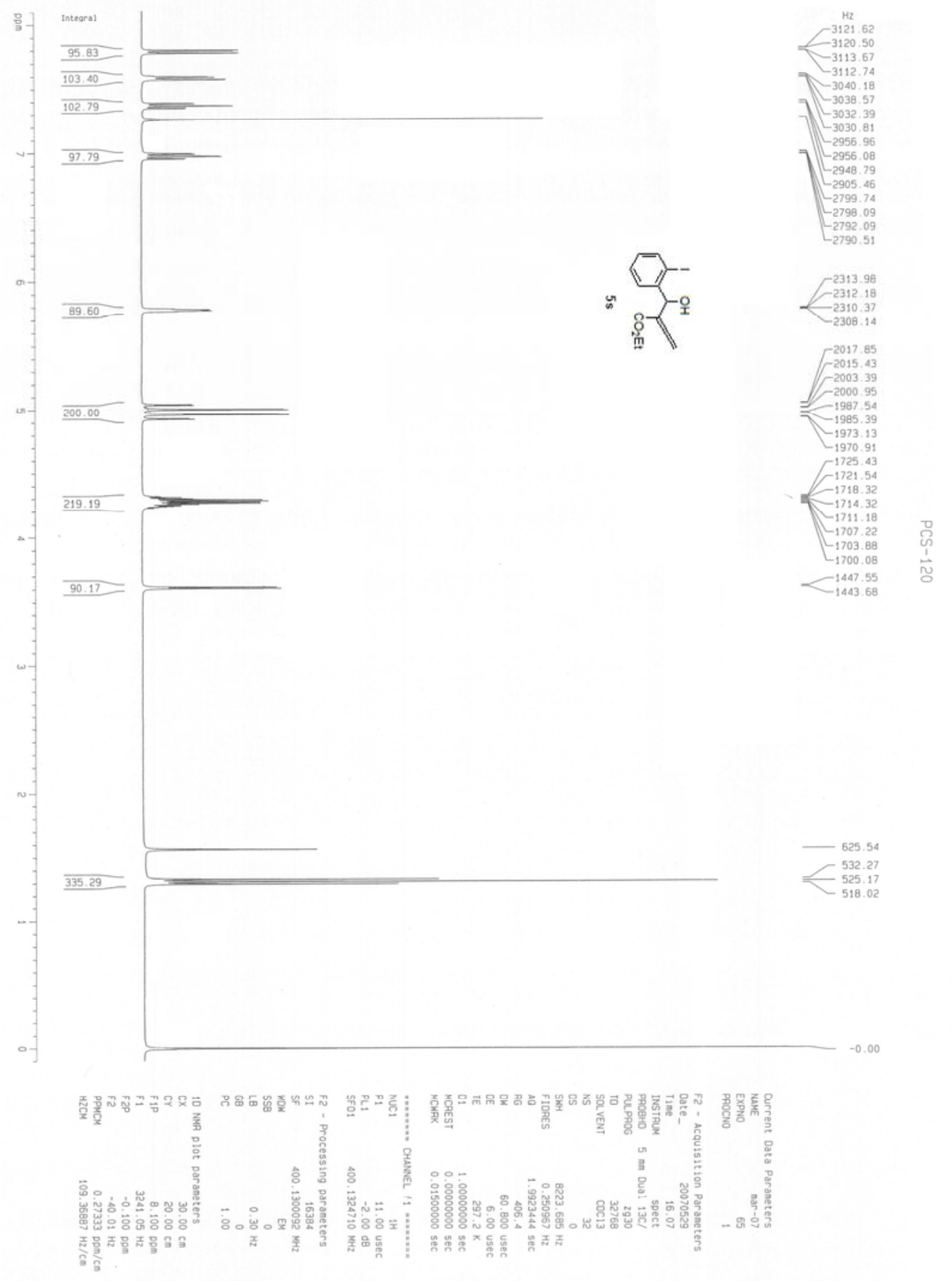



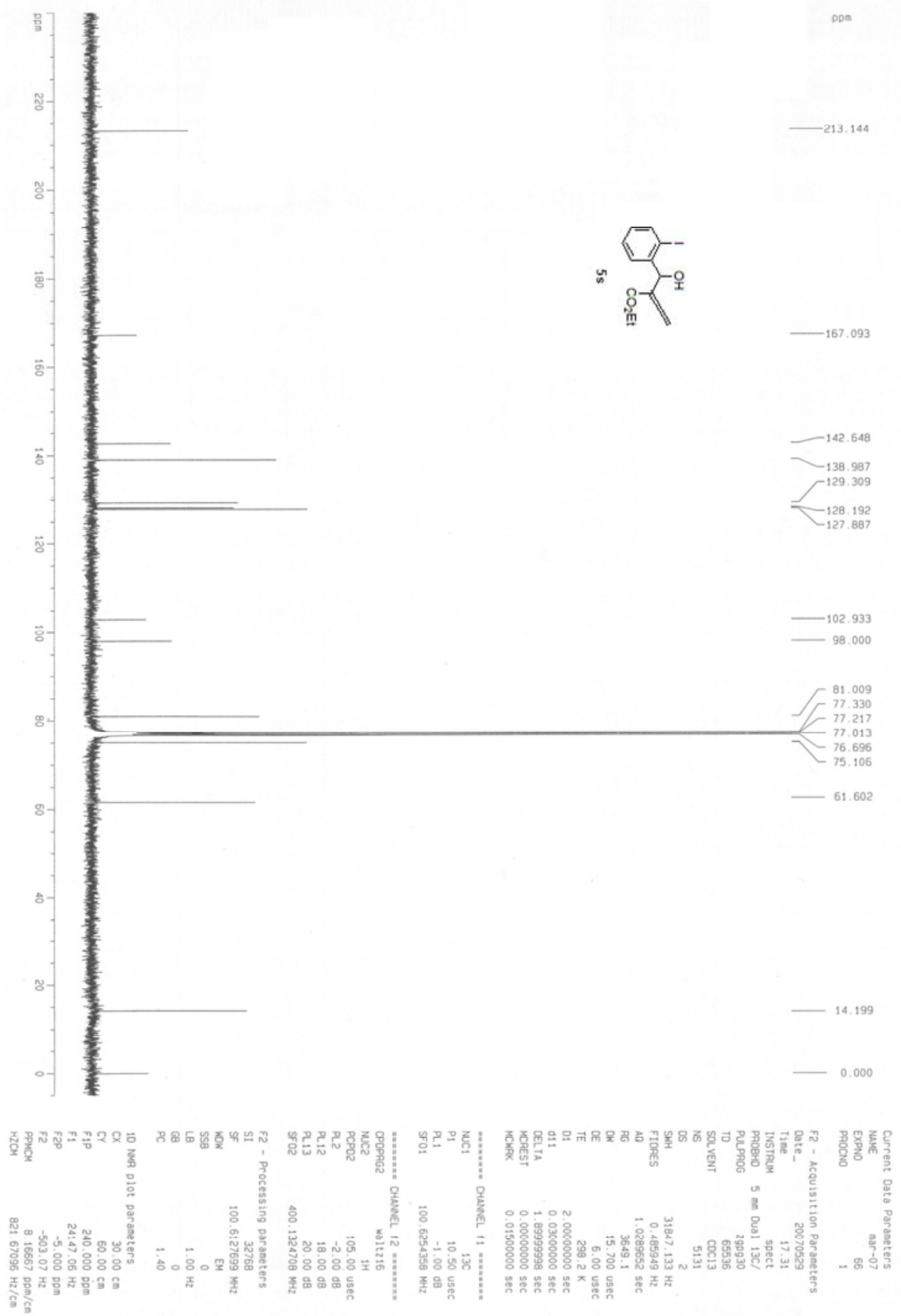


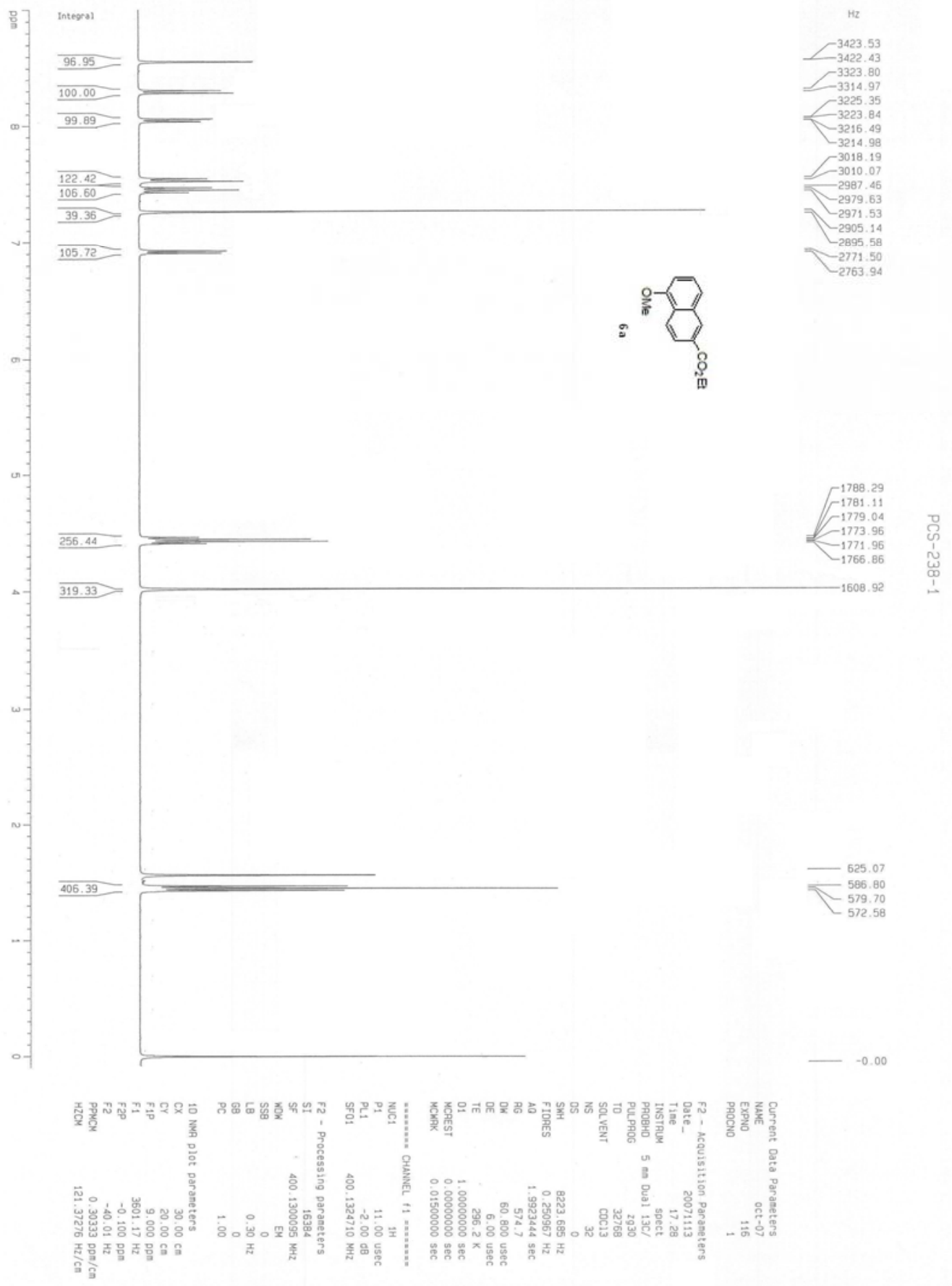



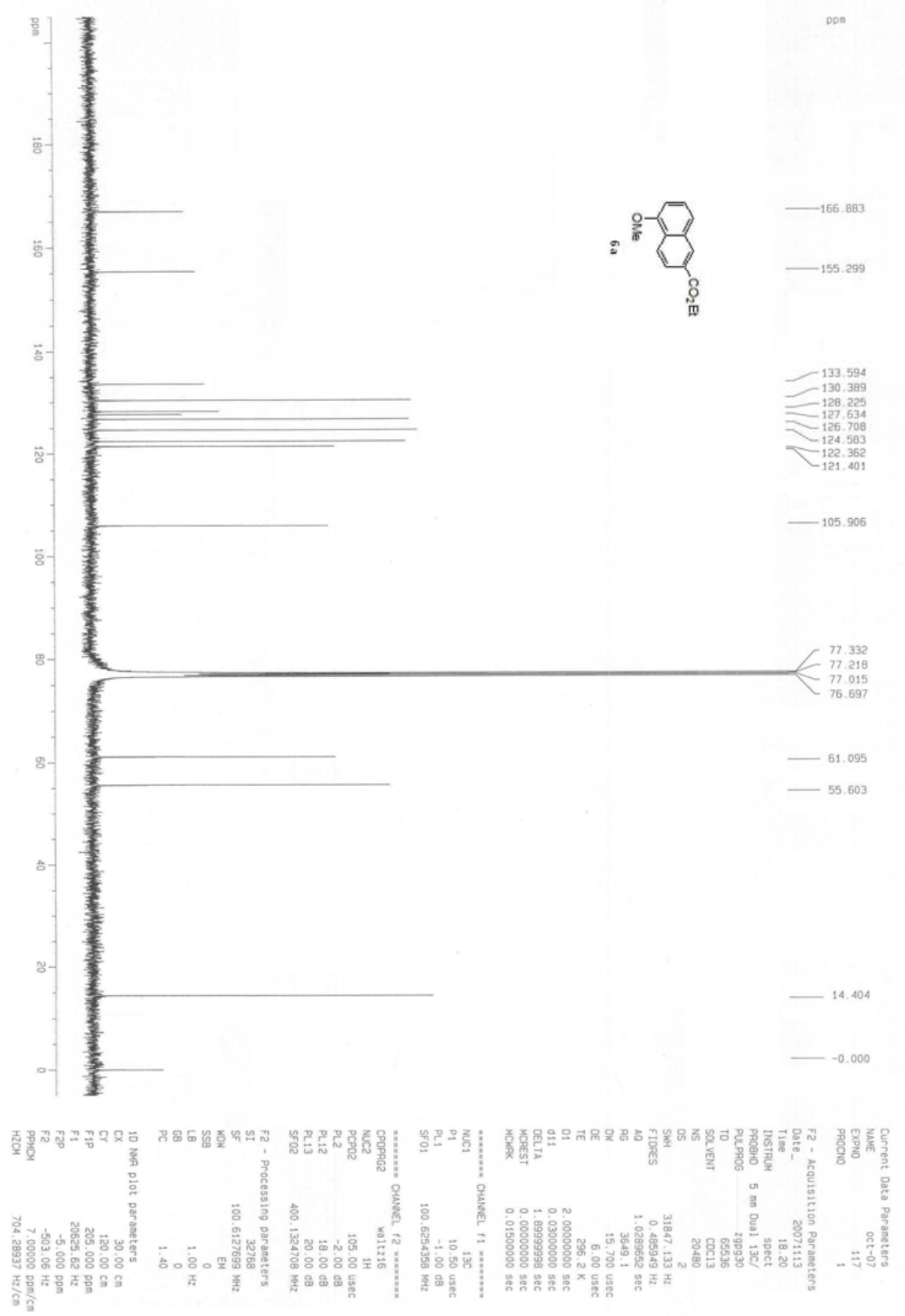


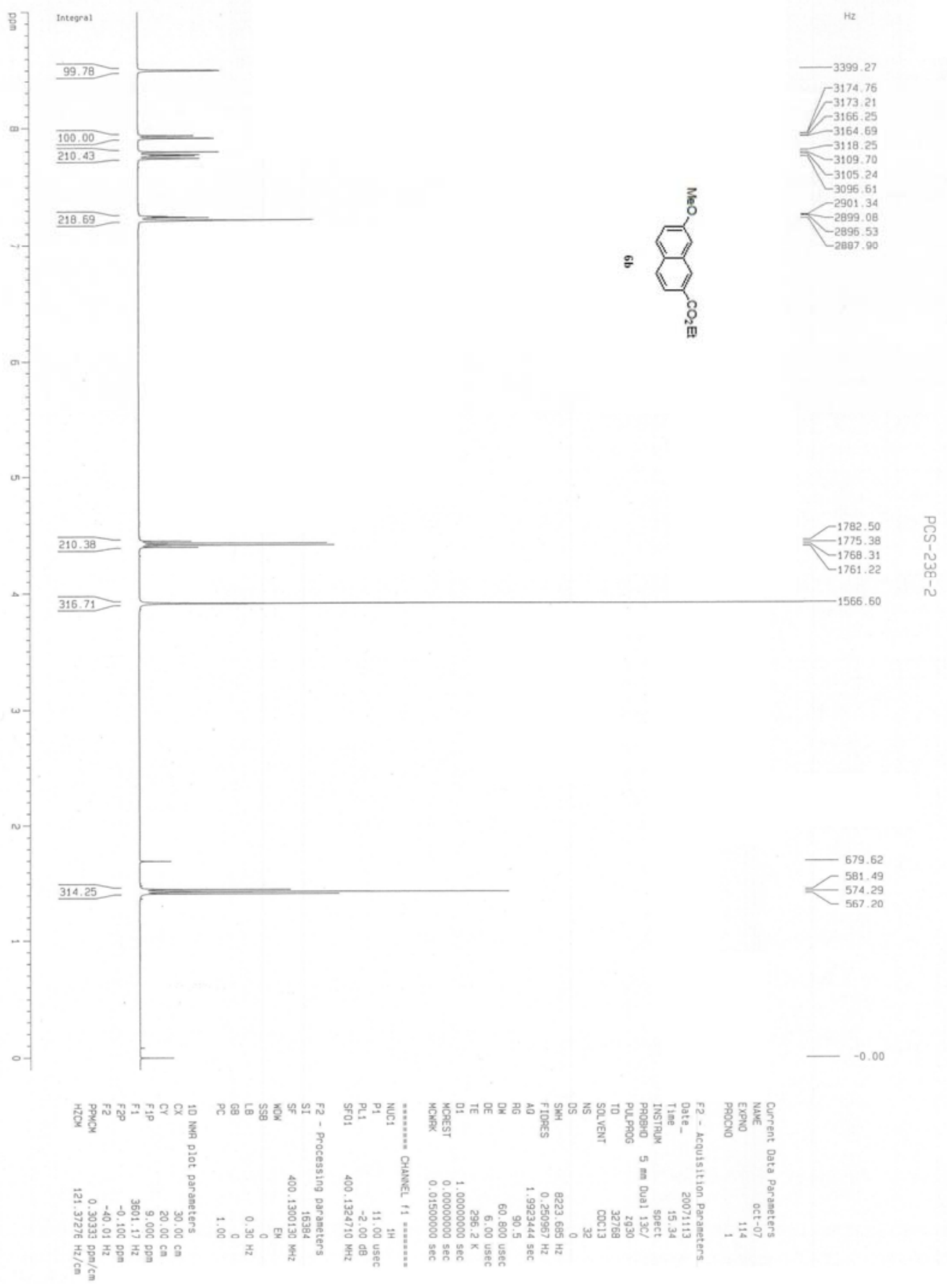



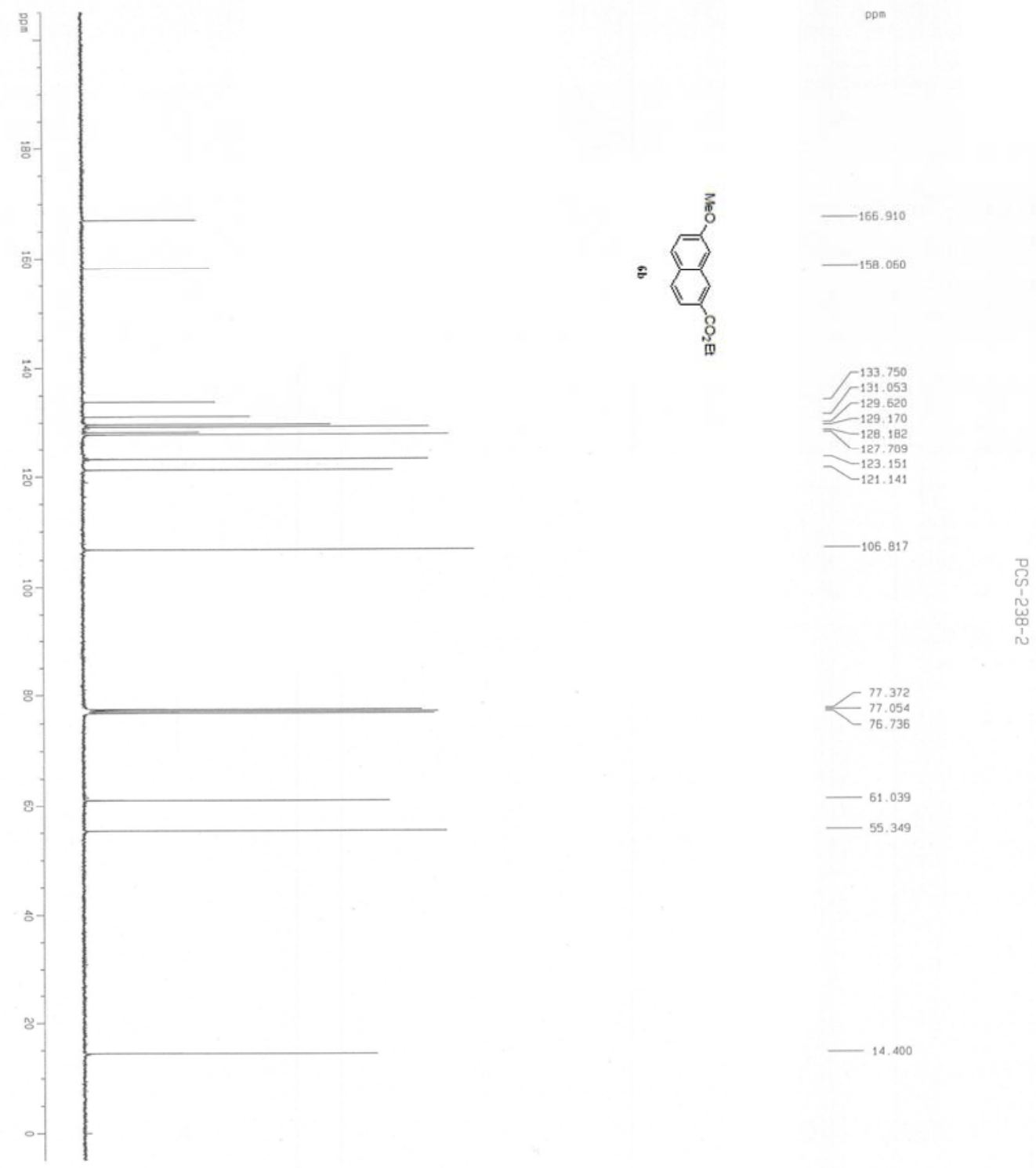

$<\begin{array}{r}77.372 \\ 77.054 \\ 76.736\end{array}$

61.039

$-55.349$

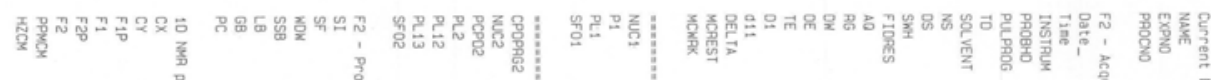

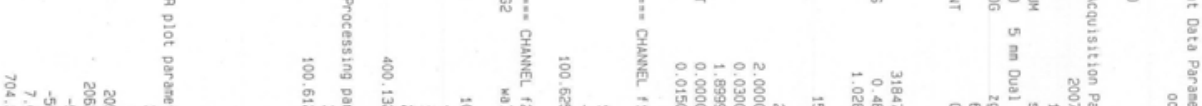

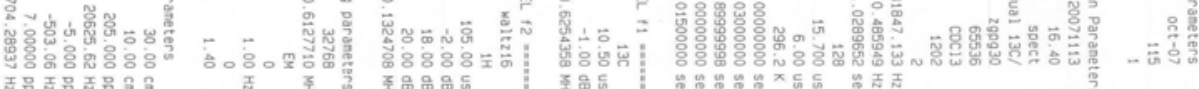

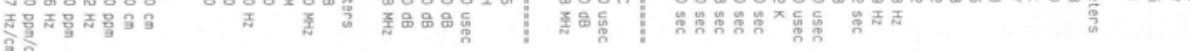




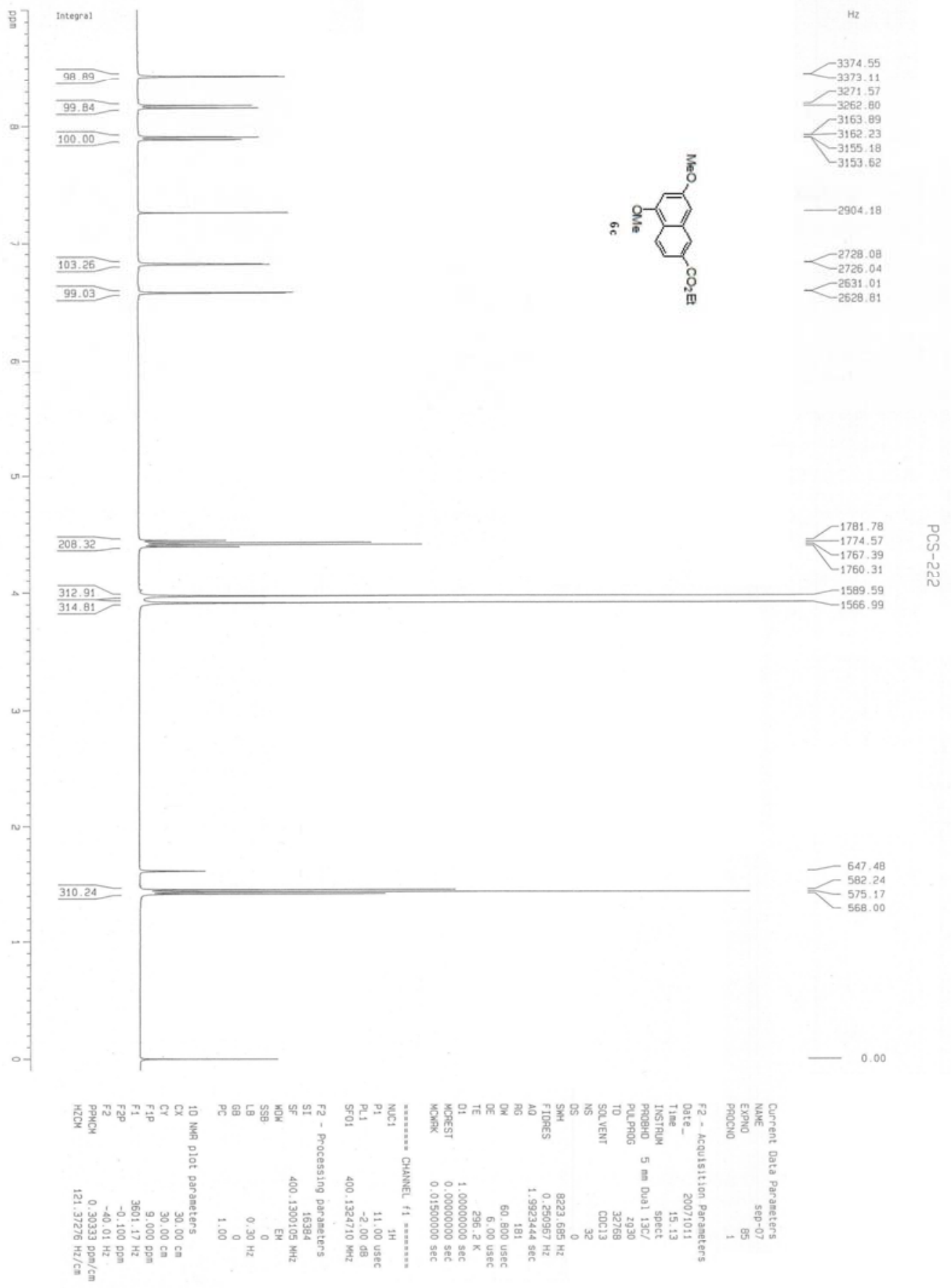




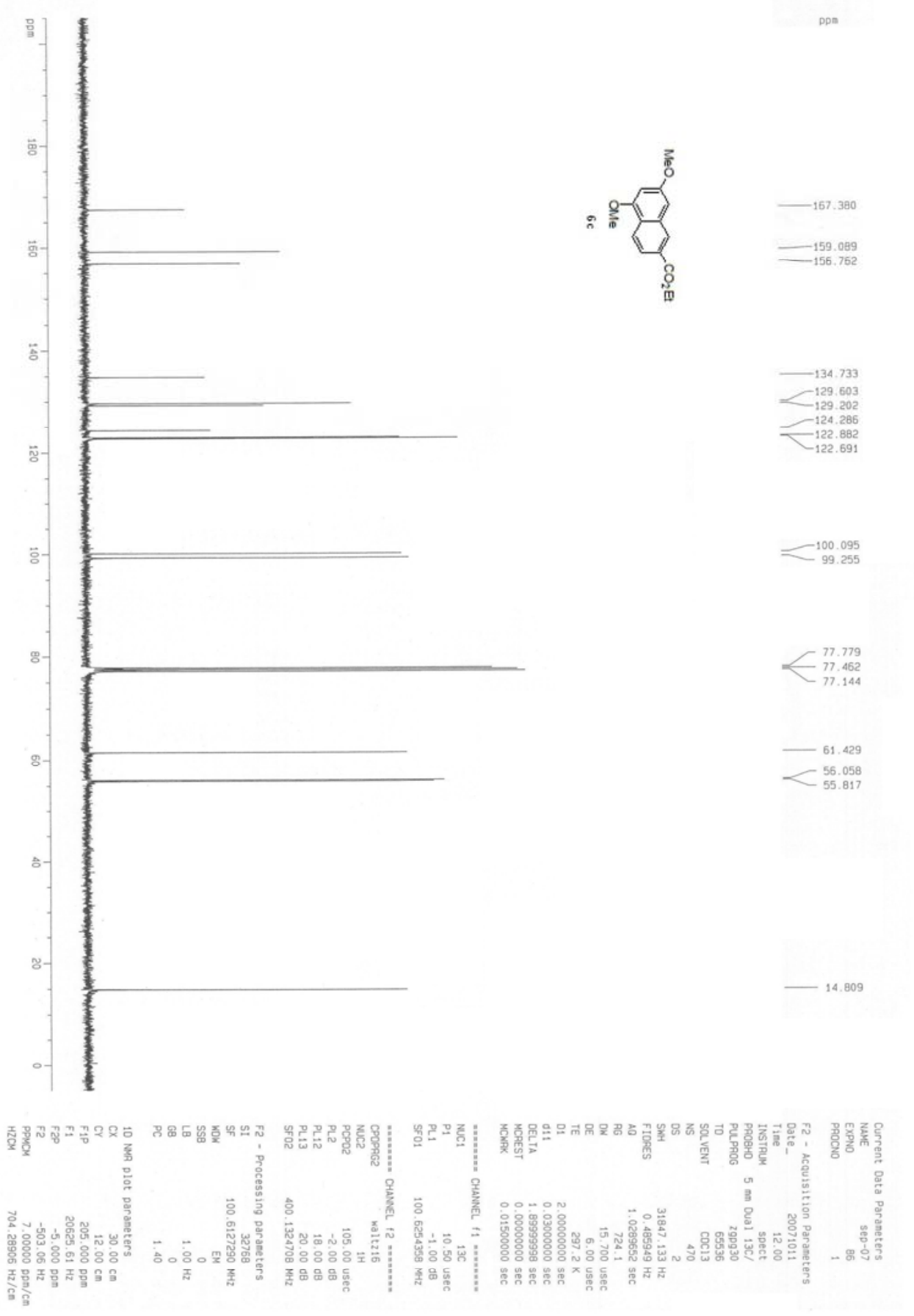




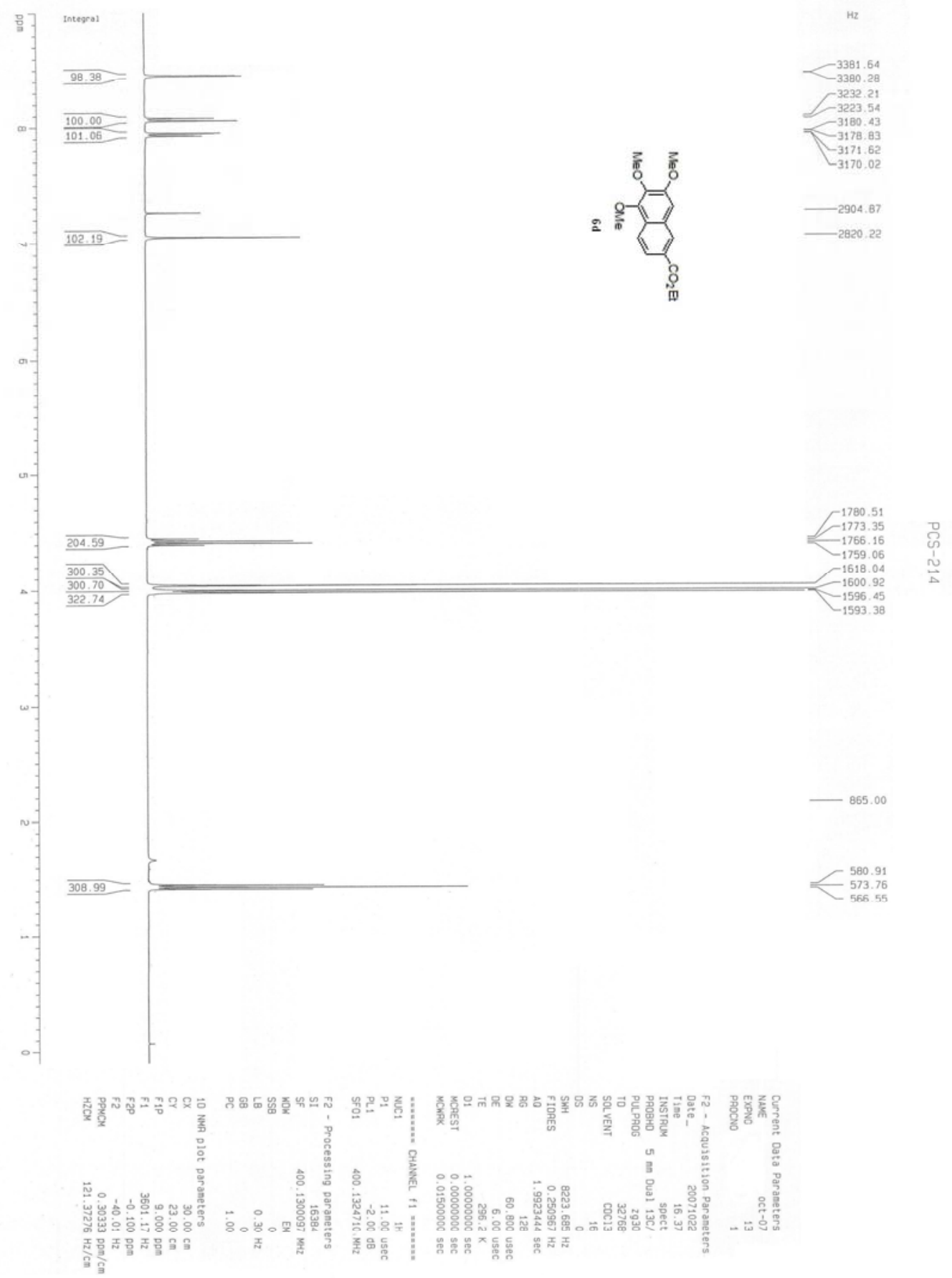




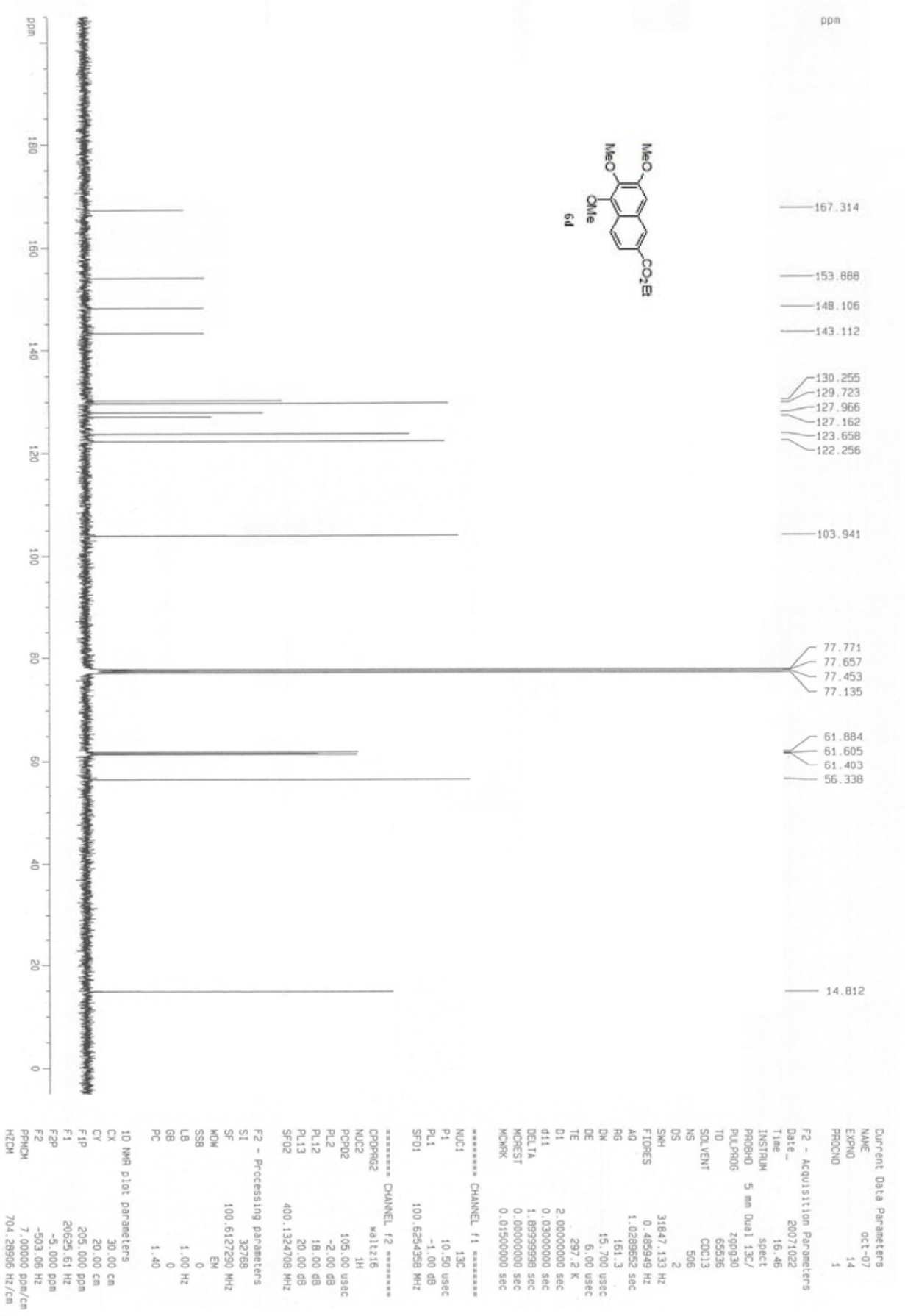




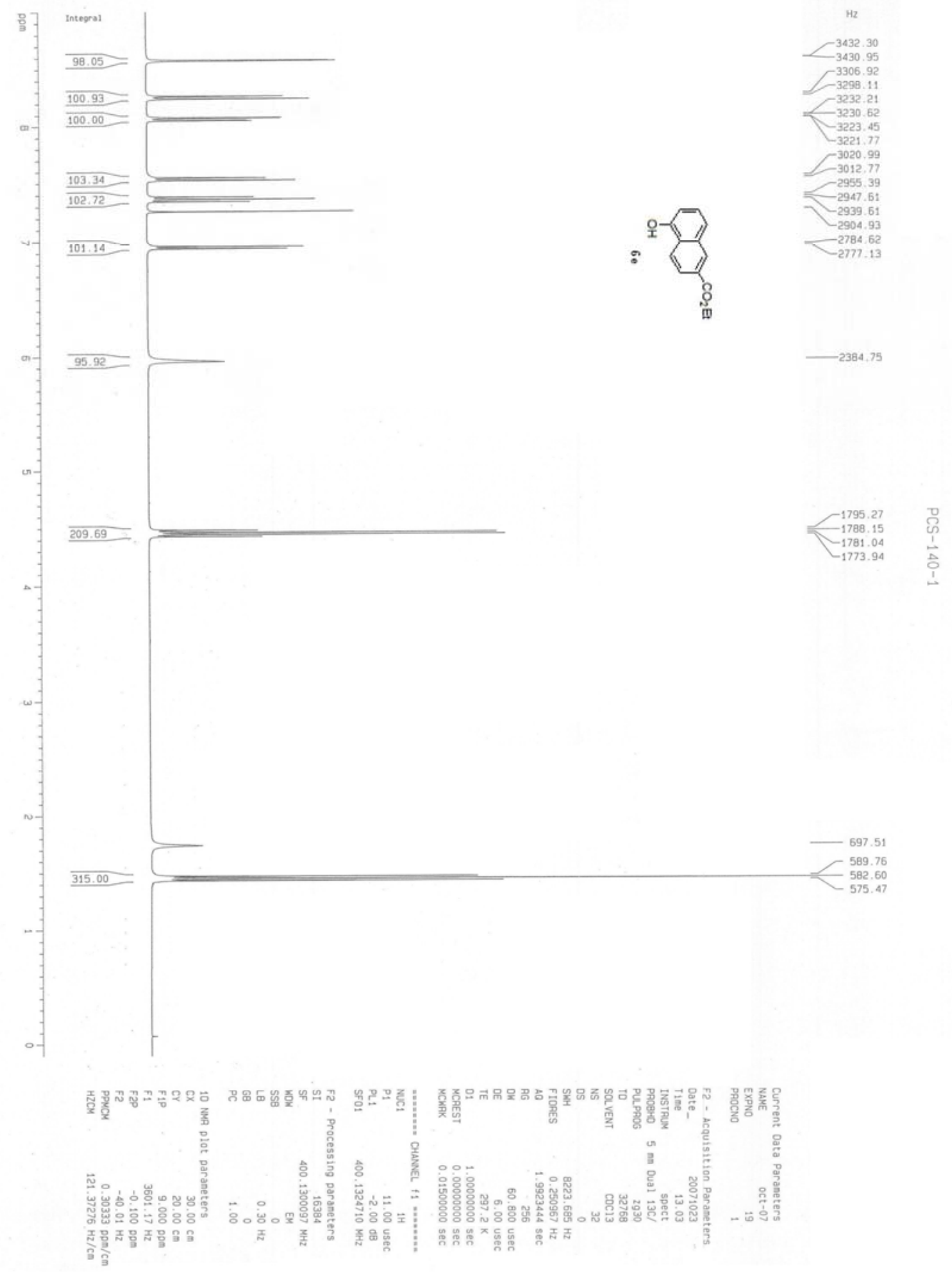




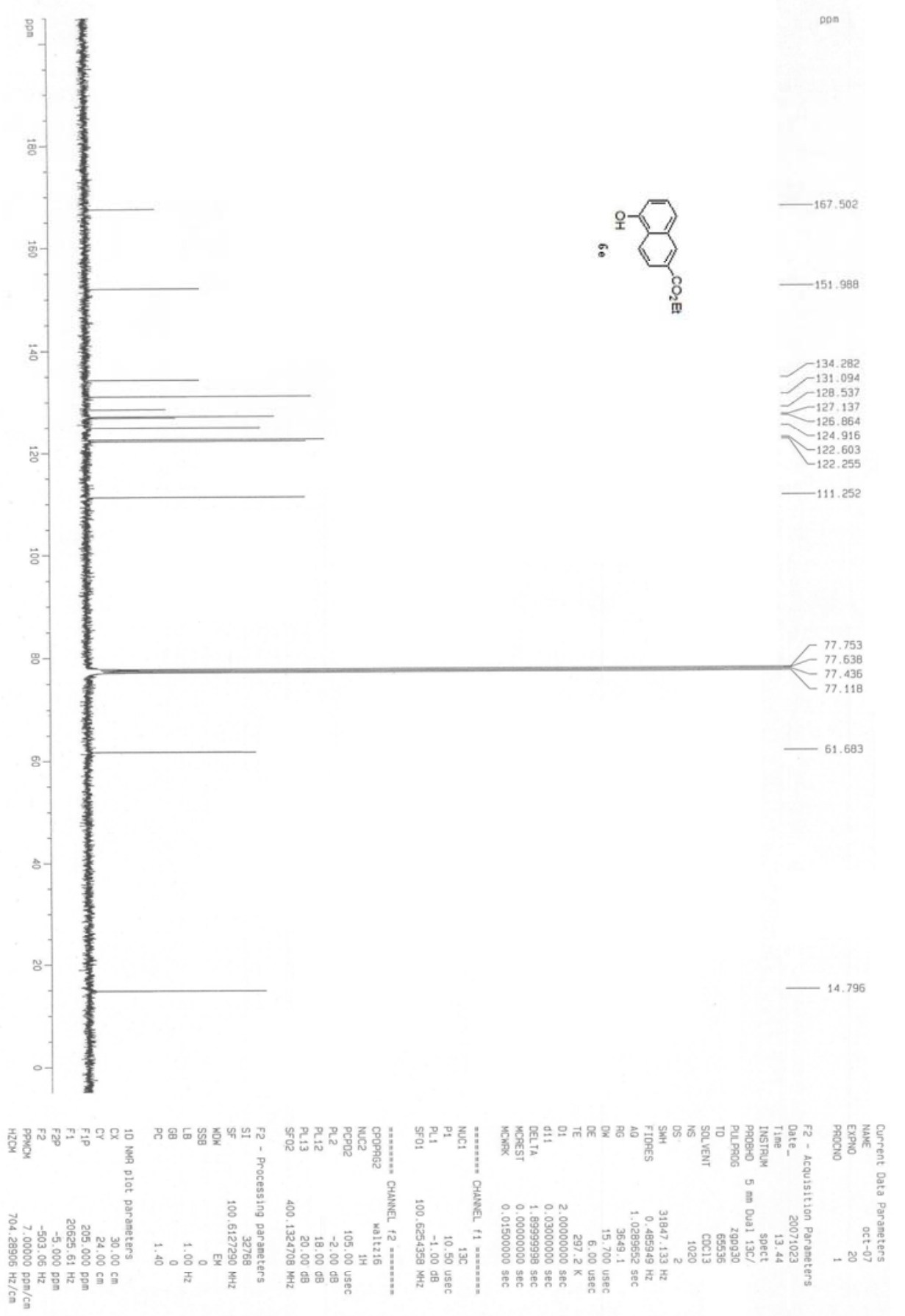




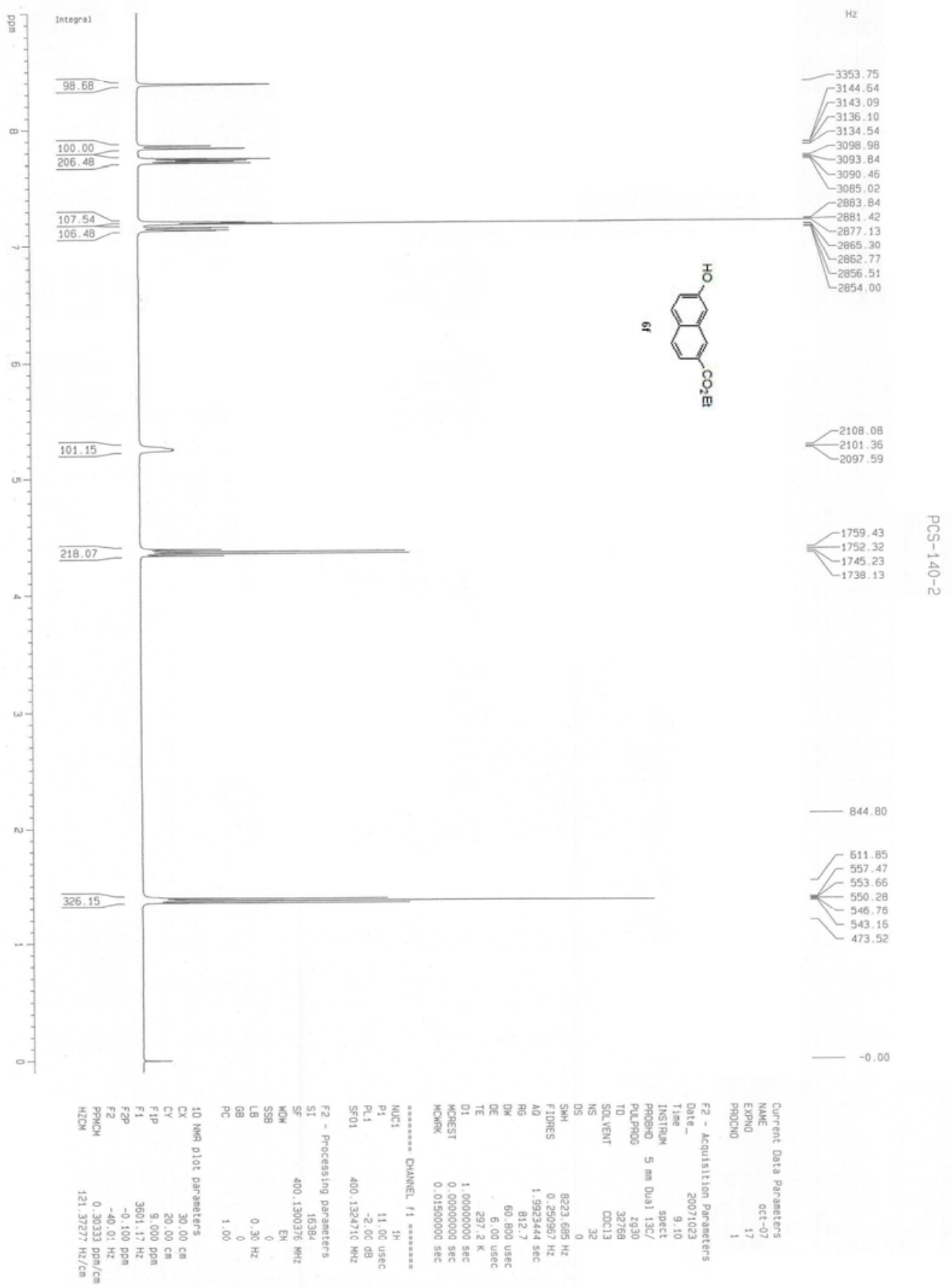




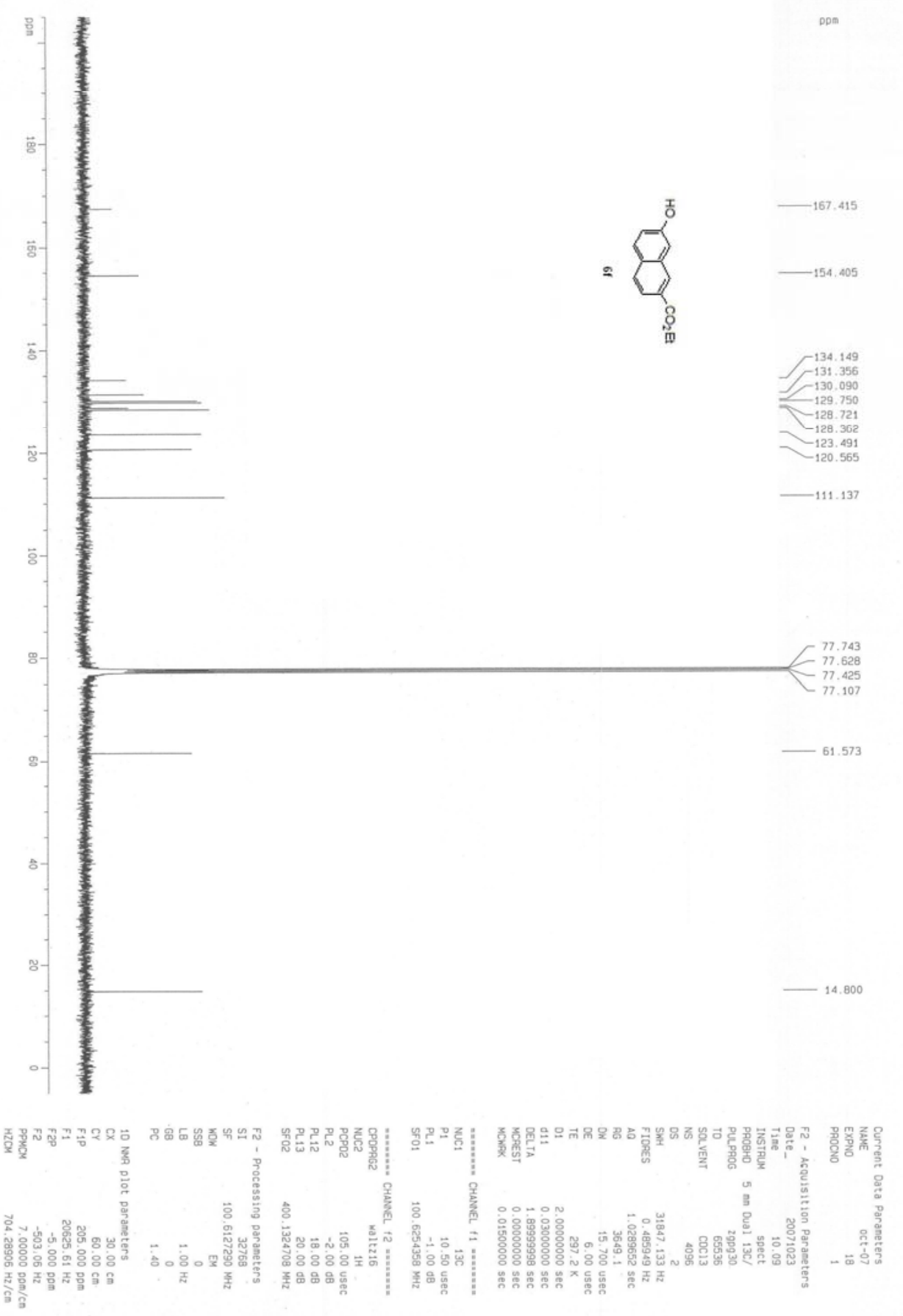




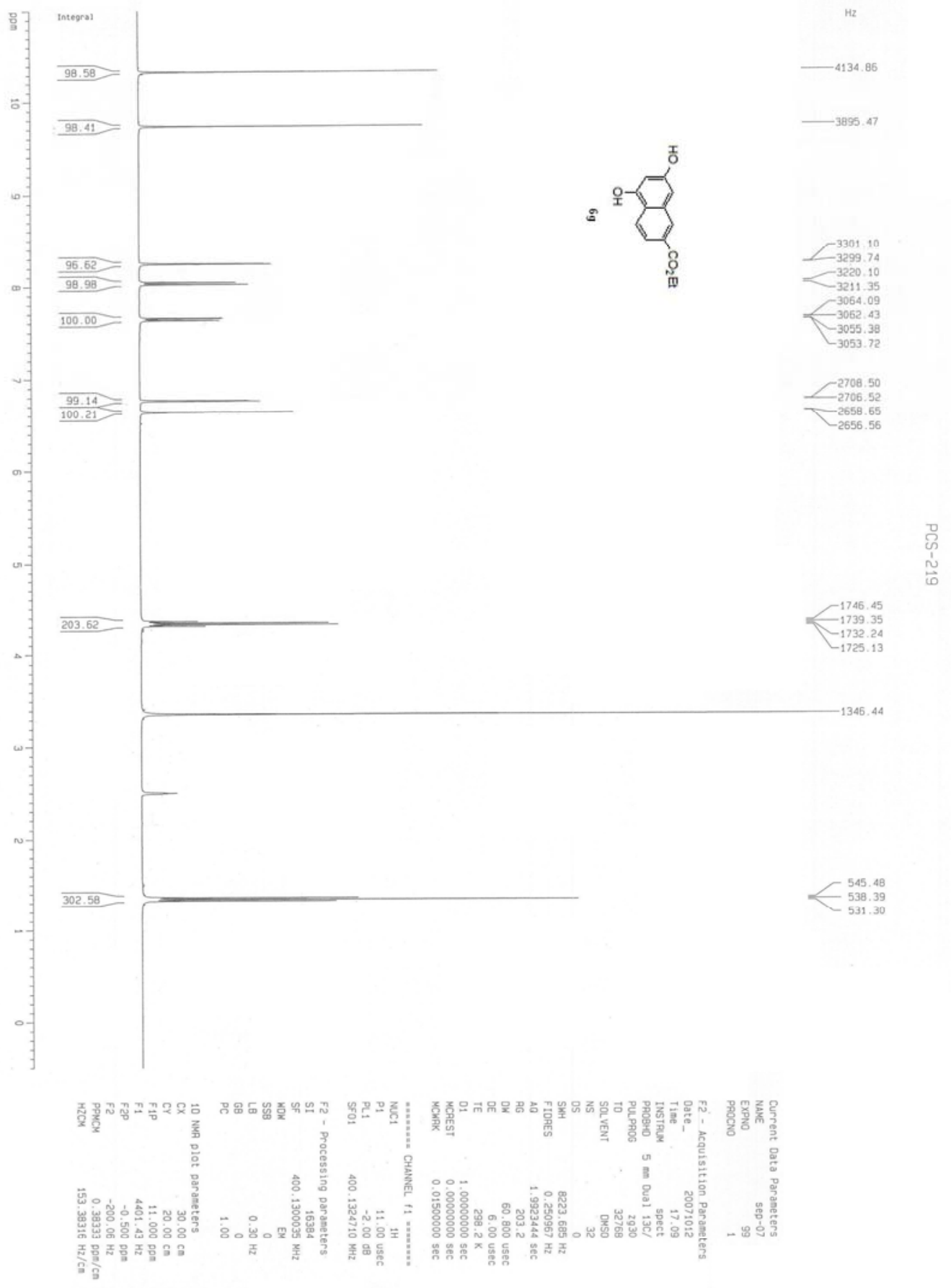




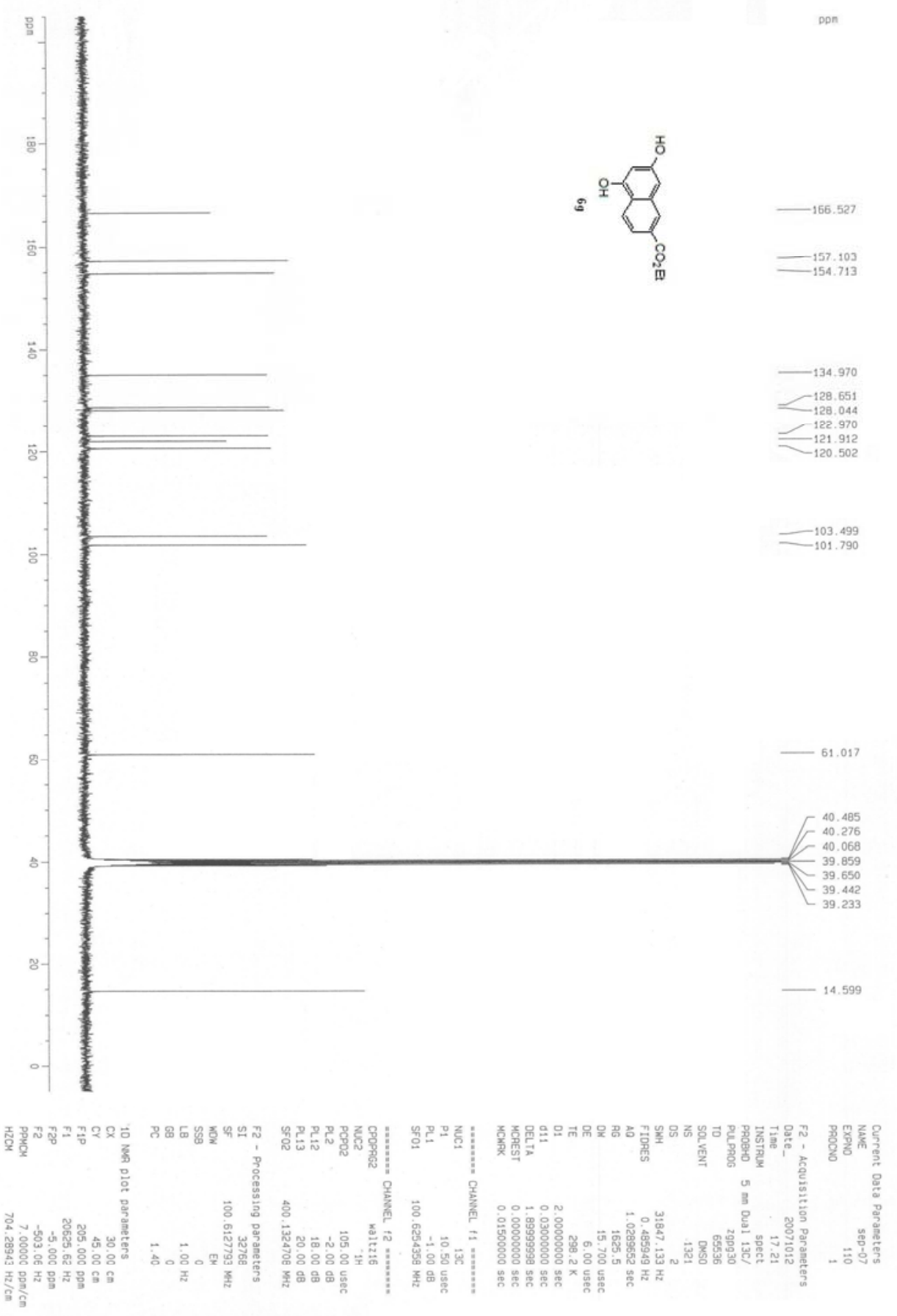

